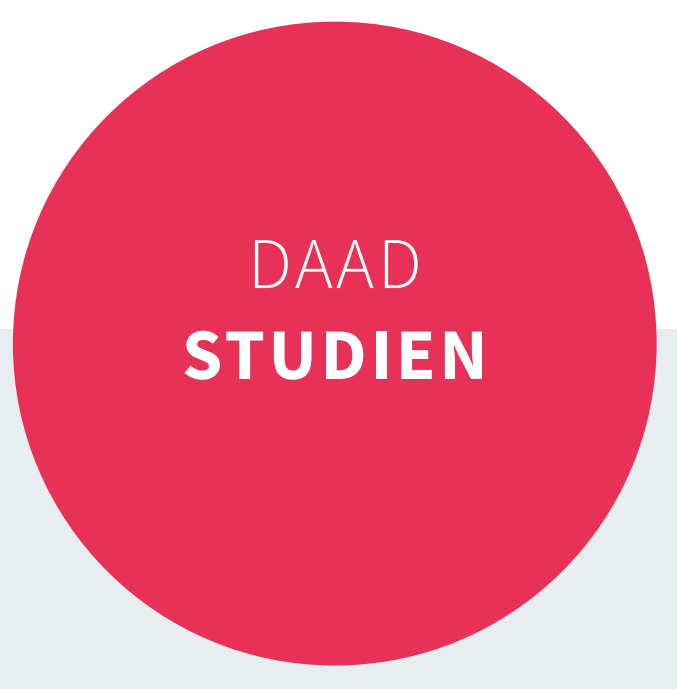

\title{
Flüchtlingsintegration und Fachkräftemigration: Welche Rolle spielen Hochschulen?
}

Begleitstudie zu den DAAD-Programmen Integra und PROFI

November 2021

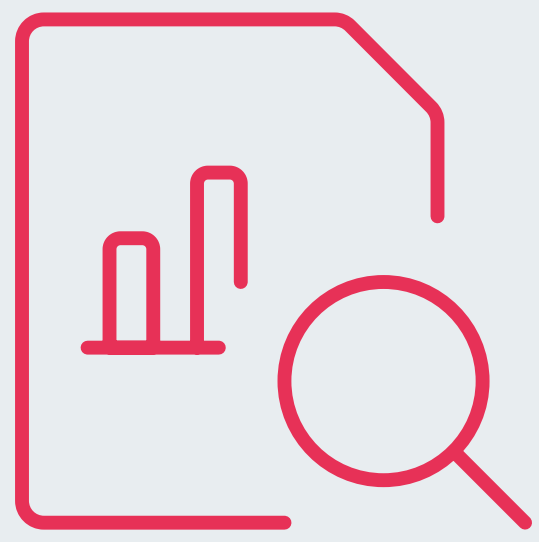

Sachverständigenrat für Integration und Migration

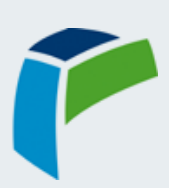

Expertise des wissenschaftlichen Stabs des Sachverständigenrats für Integration und Migration im Auftrag des DAAD 



\section{Flüchtlingsintegration und Fachkräftemigration: Welche Rolle spielen Hochschulen?}

Begleitstudie zu den DAAD-Programmen Integra und PROFI

Simon Morris-Lange

Dr. Mohini Lokhande

unter Mitarbeit von Mai-Linh Ho Dac 


\section{Inhalt}

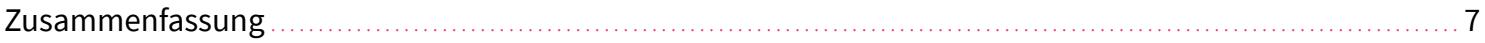

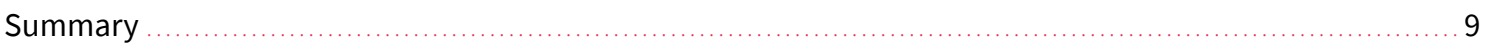

1 Fachkräftegewinnung und Flüchtlingsintegration: Neue Herausforderungen

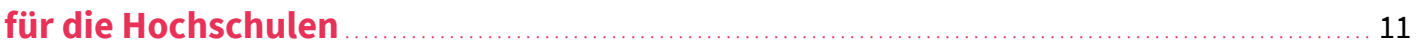

2 Zentrale Hürden auf dem Weg in die Arbeitswelt .......................................... 16

2.1 Gleichermaßen betroffen: internationale Studierende mit und ohne Fluchterfahrung ................ 18

2.2 Schwerwiegendere Hürden für ältere und berufserfahrene Zielgruppen ............................. 24

3 Wegbereitung in den Arbeitsmarkt zumeist digital: erste Erfolge und zukünftiger Entwicklungsbedarf ................................... 30

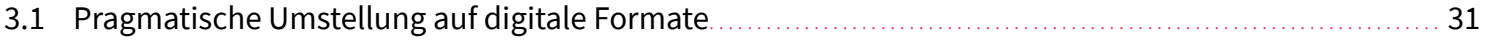

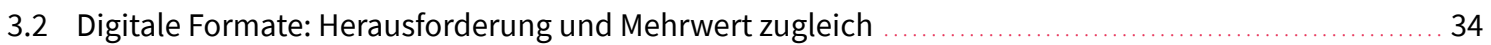

4 Noch ausbaufähig: gemeinsame Übergangsgestaltung in die Arbeitswelt .............. 38

$5 \quad$ Empfehlungen für Hochschulpraxis und -politik ........................................ 45

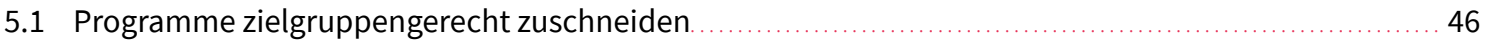

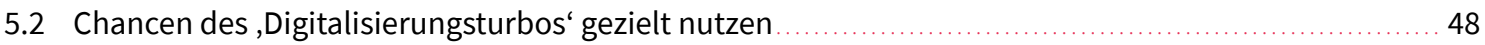

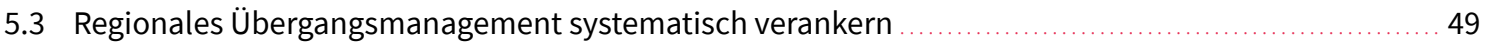

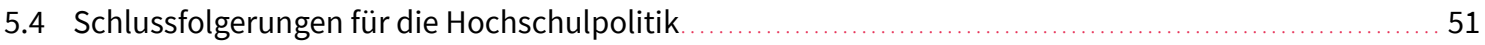

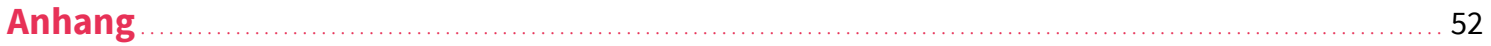

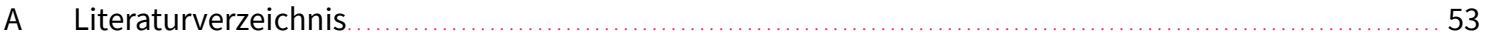

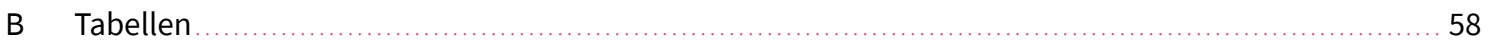




\section{Tabellen- und Abbildungsverzeichnis}

Tabelle 1: Hochschulprojekte in den Programmen Integra und PROFI 2020-2022

Tabelle 2: Altersstruktur ausgewählter Studierendengruppen an deutschen Hochschulen $\ldots \ldots \ldots \ldots \ldots \ldots . . \ldots 17$

Tabelle 3: Zugang zu Studienvorbereitung, Studium und Arbeitsmarkt für Geflüchtete .................... 20

Tabelle 4: Bewerbungen für die Teilnahme in den Integra- und PROFI-Projekten ............................ 24

Tabelle 5: Einsatz digitaler Lehr- und Lerntechnologien im Integra-Programm ............................ 33

Tabelle 6: Partnerorganisationen der Hochschulen bei der Gestaltung des Übergangs vom Studium in den Beruf

Tabelle 7 Geplante und bereits durchgeführte Kooperationsmaßnahmen mit den Partnerorganisationen.

Tabelle 8: Teilnehmende in den untersuchten Integra- und PROFI-Projekten ............................58

Tabelle 9: Ablehnungsgründe bei Bewerbungen, anteilig an projektgeförderten Hochschulen ............ 58

Tabelle 10: Arbeitsmarktvorbereitung in den untersuchten Integra- und PROFI-Projekten .................. 59

Tabelle 11: Vermittelte Übergangsmöglichkeiten in die Arbeitswelt .......................................5 59

Tabelle 12: Interview-Leitfaden für das projektverantwortliche Hochschulpersonal .........................60 60

Abb. 1 Weitverbreitete Hürden beim Berufseinstieg für ausgewählte internationale Zielgruppen an deutschen Hochschulen.

Abb. 2 Arbeitsmarktvorbereitung in Zeiten der Corona-Pandemie

Abb. 3 Anteil der Hochschulen, die den Übergang vom Studium in den Beruf gemeinsam mit externen Partnerorganisationen gestalten, nach Typus der Partner (in Prozent) 



\section{Zusammenfassung}

Das Fachkräftepotenzial der derzeit knapp 325.000 internationalen Studierenden an deutschen Hochschulen ist erheblich. Mindestens zwei Drittel beabsichtigen, nach ihrem Abschluss hierzubleiben und zu arbeiten. Allerdings ist ihr Weg in den Arbeitsmarkt mit zahlreichen Stolpersteinen gepflastert. Viele scheitern zunächst an unzureichenden Deutschkenntnissen, fehlenden beruflichen Netzwerken und zu wenig Praxiserfahrung im Inland. Ob und inwiefern diese Hürden auch den etwa 30.000 Geflüchteten unter ihnen zu schaffen machen, ist bislang kaum erforscht. Die bundesfinanzierten Hochschulprogramme Integra und PROFI, ${ }^{1}$ die der Deutsche Akademische Austauschdienst (DAAD) in den letzten Jahren für Geflüchtete entwickelt hat, erlauben nun einen ersten Einblick in die Erfahrungen dieser Zielgruppe am Übergang vom Studium in den Beruf. Die vorliegende Expertise des wissenschaftlichen Stabs des Sachverständigenrats für Integration und Migration (SVR) fasst diese Erfahrungen zusammen, vergleicht sie mit denen anderer internationaler Studierender und entwickelt Handlungsempfehlungen für die Hochschulpraxis und -politik.

Zwischen 2020 und 2022 werden bundesweit 88 Hochschulvorhaben zur Arbeitsmarktintegration von internationalen Studierenden über die Programme Integra und PROFI gefördert. Die große Mehrheit der Projektteilnehmenden ist in den letzten Jahren nach Deutschland geflüchtet. Die Projektmittel unterstützen die Hochschulen u. a. dabei, zielgruppenorientierte Bewerbungstrainings, Mentorenprogramme und Kooperationen mit der regionalen Wirtschaft aufzusetzen. Die 4.233 Studierenden im Integra-Programm durchlaufen dabei ein komplettes Studium, während die 192 Teilnehmerinnen und Teilnehmer in den PROFI-Projekten eher kürzere und branchenspezifische Weiterbildungskurse nachfragen. Sie sind meist älter und berufserfahrener als ihre Kommilitoninnen und Kommilitonen, die ein komplettes Studium absolvieren.

Die Analyse der Integra-Projekte zeigt, dass Geflüchtete beim Berufseinstieg vor sehr ähnlichen Hürden stehen wie ihre internationalen Mitstudierenden, die keine Fluchterfahrung aufweisen. Auch sie kämpfen mit sprachlichen Hürden, fehlender Praxiserfahrung, zurückhaltenden Betrieben und Schwierigkeiten beim Schreiben von Bewerbungen oder in Vorstellungsgesprächen. Die Hürden auf dem Weg in den Arbeitsmarkt sind also zunächst die gleichen. Unterschiede ergeben sich aus dem Bedarf nach zusätzlicher individueller Beratung und Betreuung, welcher i. d. R. vor allem bei Geflüchteten entsteht. Neben ihrer Fluchterfahrung erfordern ihre teils unsichere Bleibeperspektive und ihre unterbrochene Bildungsbiografie mehr Unterstützung seitens des Hochschulpersonals, z. B. beim Hochschulzugang und gegebenenfalls bei der Weitervermittlung an psychologische Dienste.

Deutlich anders gelagert sind die Herausforderungen für die Teilnehmenden in den PROFI-Projekten. Ihnen geht es nicht um eine allgemeine Arbeitsmarktvorbereitung, sondern um eine gezielte Weiterbildung. Sie benötigen ein Lernangebot, das zeitlich komprimiert und präzise abgestimmt ist auf die konkreten Anforderungen in einzelnen Zielberufen und -branchen. Daher erhoffen sie sich von den Hochschulen ein möglichst passgenaues und zeitlich begrenztes Bildungsangebot, das ihnen dabei hilft, die sprachlichen, fachlichen und technischen Erfordernisse in ihrem Wunschberuf möglichst schnell zu erfüllen. Da es sich hierbei nicht nur um Einstiegsstellen handelt, sind die Ansprüche der Betriebe und der Teilnehmenden selbst meist höher als bei frisch Graduierten.

Die Überwindung dieser und weiterer Hürden hängt in erster Linie vom individuellen Engagement der Lernenden ab. Doch es gibt zahlreiche Unterstützungsangebote: In PROFI und Integra können Programmteilnehmende auf zusammengerechnet mehr als 500 Einzelangebote zum Arbeitsmarkteinstieg zurückgreifen.

1 Integra = Integration von Flüchtlingen ins Fachstudium; PROFI = Programm zur Förderung der bildungsadäquaten Integration in den deutschen Arbeitsmarkt. 
Etwas mehr als die Hälfte dieser Unterstützungsleistungen wurde in der laufenden Förderphase neu entwickelt. Neben Bewerbungsmappen-Checks und Bewerbungsworkshops setzen die Hochschulen vor allem auf zielgruppenorientierte Seminare, etwa zur Berufspraxis in ausgewählten Branchen, oder auf Gastvorträge durch Unternehmen, freiberufliche Karrierecoaches und andere Partnerinnen und Partner. All diese Angebote gehen gezielt auf den zusätzlichen Unterstützungsbedarf Geflüchteter ein, die häufig noch nicht oder nur unzureichend mit den Gepflogenheiten auf dem hiesigen Arbeitsmarkt vertraut sind. Aufgrund der andauernden Corona-Pandemie wurden über 90 Prozent der Unterstützungsangebote in den digitalen Raum verlagert und über Videokonferenzen, digitale Lernplattformen und andere Technologien realisiert. Durch die kurzfristige Digitalisierung konnte ein Großteil des Angebots aufrechterhalten werden, i. d. R. durch den pragmatischen Einsatz von Videokonferenzen. Gleichzeitig stehen aber eine Evaluation und eine didaktische Weiterentwicklung vielerorts noch aus. Von letzterer könnten Geflüchtete sehr profitieren, da sie einerseits oft weniger Vorerfahrung mit digitalen Lernplattformen und Lerntechnologien haben als diejenigen, deren Studium in Deutschland länger im Voraus geplant war. Andererseits erfordern digitale Lernformate eine hohe Lese- und Schreibkompetenz sowie ein gutes sprachliches Hörverständnis, was die ohnehin schon bestehenden sprachlichen Hürden für diejenigen verschärft, die Deutsch bzw. Englisch (noch) nicht auf muttersprachlichem Niveau beherrschen.

Neben den Unterstützungsangeboten der Hochschulen bedarf es einer verstärkten Zusammenarbeit derselben mit Unternehmen, Kammern, der Agentur für Arbeit und anderen regionalen Partnerorganisationen, damit Bleibewillige erfolgreich in den Arbeitsmarkt einmünden können. Insgesamt berichten die Projektverantwortlichen an den Hochschulen von mehr als 600 Partnerorganisationen, mit denen sie eine Zusammenarbeit im Zuge der Programmförderung anstreben. Durchschnittlich sind dies etwa sieben pro Hochschule. Bislang zeigt sich, dass die Zusammenarbeit in den allermeisten Fällen bilateral und anlassbezogen erfolgt, z. B. im Rahmen von Gastvorträgen oder einer Verweisberatung. Das hat Vorteile: Sowohl die einzelne Hochschule als auch die Partnerorganisation verfügen dabei über einen konkreten Grund für eine Zusammenarbeit. Die Kooperation ist somit vergleichsweise niedrigschwellig umsetzbar, insbesondere dann, wenn sie die individuellen Stärken der Beteiligten gezielt nutzt. Der Nachteil dieser und weiterer bilateraler Verbindungen in den Arbeitsmarkt ist, dass vereinzelte Kooperationen zwischen Hochschulen und Unternehmen bzw. sonstigen Partnerorganisationen oft unverbunden nebeneinanderstehen und nur einen Teil des gesamten Übergangsgeschehens abbilden. Hochschulen, die sich allein auf bilaterale Formen der Zusammenarbeit verlassen, erhöhen somit nicht nur die Gefahr von Doppelstrukturen, auch die Breitenwirkung ihrer Einzelvorhaben ist deutlich geringer als bei einer Kombination aus bilateralen und multilateralen Kooperationen.

Damit der Berufseinstieg von geflüchteten und anderen internationalen Studierenden in Zukunft besser gelingt, bedarf es insbesondere

- eines zielgruppengerechten Zuschnitts der Fördermaßnahmen, d. h. gemeinsame Angebote für internationale Studierende mit und ohne Fluchterfahrung sind durch punktuelle Zusatzangebote für internationale Teilnehmerinnen und Teilnehmer an hochschulischer Weiterbildung sowie, je nach Bedarf, zusätzliche individuelle Beratung und Betreuung zu ergänzen,

- einer gezielten Weiterentwicklung skalierbarer digitaler und hybrider Hilfsangebote, die neben dem konsequenten Auf- und Ausbau der digitalen Infrastruktur und Kompetenzen - sowohl bei den Lernenden als auch beim Personal - auch eine didaktische Anpassung an die sprachlichen und lernkulturellen Ausgangslagen internationaler Zielgruppen umfasst,

- einer systematischen Wegbereitung von der Studien- in die Arbeitswelt in Form eines regionalen Übergangsmanagements, das die zahlreichen Einzelinitiativen von Hochschulen, Unternehmen, der Arbeitsvermittlung und weiterer regionaler Partnerorganisationen an den jeweiligen Standorten miteinander verzahnt und so den internationalen Fachkräften von morgen klare Wege in den (regionalen) Arbeitsmarkt aufzeigt. Von einem solchen multilateralen Brückenschlag zwischen Studium und Beruf könnten auch deutsche Studierende profitieren. 


\section{Summary}

There are around 325,000 international students at German institutions of higher education. These students could play a potentially important role in reducing the shortage of skilled workers in Germany. At least two thirds of these students wish to remain and work in Germany after graduating. Policymakers also increasingly see international students who wish to stay in Germany as ideal candidates to fill the growing number of job vacancies. Nonetheless, there are many obstacles to their entry into the labour market. Many simply do not speak good enough German or have the professional networks and experience they need. There is very little research on whether and to what extent these obstacles pose problems for the around 30,000 refugees among these international students. In recent years the German Academic Exchange Service (DAAD) has used federal funding to develop its Integra and PROFI academic qualification programmes ${ }^{2}$ for refugees. These programmes now provide some initial insights into how this target group is managing the transition from university to the labour market. The expert report produced by The Expert Council on Integration and Migration (SVR) summarises these experiences, compares them with those of other international students and develops recommendations for higher education practice and policy.

Between 2020 and 2022, 88 institutions of higher education will receive Integra and PROFI funding for projects designed to integrate student refugees and other international students in the labour market. These temporary project grants will enable universities to cooperate with regional businesses and to implement measures such as job application training and mentoring programmes which are tailored to the needs of refugees and other international students. The 4,233 students in the Integra programme will initially complete a full degree programme, while the 192 participants in PROFI projects are more likely to choose shorter continuing education courses relating to specific areas of work. Most of the participants are older and have more work experience than those of their Integra peers.

The analysis of the Integra projects shows that the obstacles that refugees face entering the labour market are very similar to those confronting other international students. Both groups struggle with language barriers, lack of professional experience, reluctance on the part of employers and difficulties in writing applications or coping with interviews. In other words, both groups initially face the same hurdles to entering the labour market. The difference between the two groups is that refugees usually require more individual counselling and support services. Refugees are not only likely to have money worries, many of them do not know whether they will be allowed to stay in Germany and may have fragmented educational biographies. For reasons such as these they require more support from higher education professionals, such as with claiming financial aid (BAföG) or with referrals to psychosocial services.

The challenges facing participants in the PROFI projects are very different. The participants in these projects need very specific continuing education courses rather than general preparation for the labour market. They require opportunities to learn in a short space of time and in a form geared to the requirements of specific professions and industries. These participants are consequently looking for compact university-based courses that will enable them to meet the language, professional and technical requirements specific to their desired profession as quickly as possible. As these participants are not only applying for entry-level positions, both their expectations and those of employing firms are in most cases higher than would be the case with recent graduates.

2 Integra = Integrating Refugees in Higher Education; PROFI = Programme Promoting Professional Integration of Academics with a Refugee Background in the German labour market 
In order to overcome these and other obstacles, participants in PROFI and Integra programmes can choose from over 500 measures designed to facilitate entry into the labour market. Most of these measures were developed from scratch during the current funding period. These include checks on CVs and cover letters as well as application workshops. In addition, higher education institutions concentrate on running tailored seminars on topics such as professional experience in specific industries or organise guest lectures by companies, freelance career advisers and other partners. Due to the ongoing Covid-19 pandemic, more than 90 percent of these supporting measures are currently being offered in the form of video conferences, on digital learning platforms or using other technologies. The speedy adoption of digital technologies has meant that most measures continue to be offered, usually by making pragmatic use of video conferences. Nonetheless, in many cases measures have not yet been either evaluated or didactically adjusted to on-screen learning. This would be beneficial for refugees. On the one hand, this group of students often has less previous experience with digital learning platforms and technologies than those able to plan their studies in Germany well in advance. On the other, digital learning formats require a high level of reading and writing skills as well as good listening comprehension. These are even tougher obstacles for students who have not (yet) acquired native-level German or English language skills.

Universities not only need to provide support, they also need to cooperate more closely with businesses, chambers of commerce, the Federal Employment Agency and other local partners to enable international students to enter the labour market. University project officers report a total of over 600 partner organisations they wish to work with using project funding. This is an average of seven per university. So far cooperation has in most cases been bilateral and event based, e.g., as part of guest lectures or referrals for counselling or advice. The advantages are that individual universities and partner organisations have specific reasons for collaborating. This reduces the threshold for cooperation, particularly if use is made of participants' individual strengths. The disadvantage of these and other bilateral links to the labour market is that sporadic cooperation between universities and companies or other partner organisations is often uncoordinated and represents only one aspect of the overall transition from study to work. Universities which rely exclusively on bilateral forms of cooperation thus run a higher risk of creating duplicate structures. What is more, the broad impact of their specific projects is significantly lower than it would be if bilateral and multilateral forms of cooperation were combined.

In order for refugees and other international students to transition into the labour market more successfully in the future, there is a particular need for

- targeted support programmes, i.e., measures which are designed for all international students, whether or not they have come to Germany as refugees. There is a need for additional measures in certain areas for those taking part in continuing education programmes and, in some cases, for additional individual counselling;

- more advanced digital and hybrid supporting measures that develop and expand the digital infrastructure and skills of learners and staff. Didactically these should be take note of the language skills and heterogeneous learning styles of international target groups;

- systematic support for the transition to work through a local job entry support scheme that links up the many initiatives being run by universities, companies, employment agencies and other cooperating regional organisations. Such support can guide the international talent towards (regional) labour markets. German students could benefit as well from such multilateral bridge-building between the world of study and the world of work. 


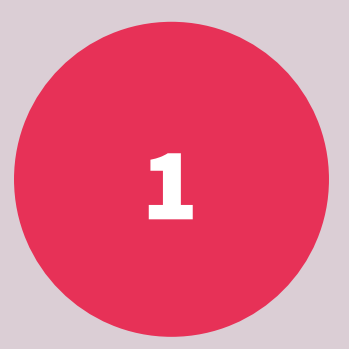

\section{Fachkräftegewinnung und Flüchtlingsintegration: Neue Herausforderungen für die Hochschulen}

Verantwortlich für die Erstellung dieser Begleitstudie ist der wissenschaftliche Stab des SVR. Die Argumente und Schlussfolgerungen spiegeln nicht notwendigerweise die Meinung des
SVR wider. Die Studie wurde im Auftrag des Deutschen Akademischen Austauschdienstes erstellt. Das Autorenteam dankt Malte Götte und Eva Wübben für ihre Unterstützung im Projekt. 
Mittlerweile herrscht grundsätzlich Einigkeit darüber, dass Deutschland in den kommenden Jahrzehnten demografiebedingt einen Bevölkerungsrückgang und somit deutlich verschärfte Fachkräfteengpässe verzeichnen wird. Zwar kann die Bundesrepublik dem demografischen Wandel u. a. mit gezielter (Nach-)Qualifizierung und einer erhöhten Erwerbsbeteiligung von Frauen und Älteren teilweise begegnen, doch selbst optimistische Prognosen gehen davon aus, dass neben der EU-Binnenwanderung in erheblichem Maße auch Zuwanderung aus Drittstaaten notwendig ist, um den Bedarf an Fachkräften zu decken (Sachverständigenrat Wirtschaft 2017: 387-388; 2020: 259; Burstedde et al. 2021: 20). Im Zuge dessen hat sich in den vergangenen Jahren der Blick auf internationale Studierende ${ }^{3}$ geändert: Sie werden nicht mehr nur als Studierende betrachtet, sondern zunehmend als Idealbesetzung für die wachsende Zahl offener Arbeitsstellen (siehe u. a. BMBF 2017: 30; Bayerischer Landtag 2020: 1-2; BDA/HRK/ BDI 2014: 3). ${ }^{4}$ Den Hochschulen wird bei der Förderung ihres Verbleibs eine wegbereitende Rolle zugeschrieben, wenngleich die bisherige Hochschulpraxis wie auch die einschlägigen politischen Strategien und Pilotvorhaben noch kein einheitliches Vorgehen erkennen lassen. ${ }^{5}$

Das Fachkräftepotenzial von internationalen Studierenden ist erheblich: Im Jahr 2021 lernen rund 325.000 internationale Studierende an deutschen Hochschulen. Über 90 Prozent von ihnen streben hierzulande einen Abschluss an und mindestens zwei Drittel beabsichtigen, nach dem Studium in Deutschland zu bleiben, um hier zu arbeiten (Statistisches Bundesamt 2021a; DAAD/DZHW 2021; SVR-Forschungsbereich 2017a: 16). ${ }^{6}$ Selbst die andauernde Corona-Pandemie und die damit einhergehenden Einreisebeschränkungen haben das Interesse an einem Studium in Deutschland bislang nicht geschmälert. Die Zahl der internationalen Studierenden ist sogar von insgesamt 319.902 vor Pandemiebeginn auf 324.729 im Wintersemester 2020/2021 gestiegen. Dieser Zuwachs ist allerdings womöglich auf Studieninteressierte zurückzuführen, die sich bereits im Inland befanden, insbesondere solche aus Indien und Syrien. Die Anzahl der Studierenden aus beiden Ländern jedenfalls ist entgegen dem allgemeinen Nullwachstum um 15 bzw. 6 Prozent angestiegen. ${ }^{7}$ Der überwiegende Teil der syrischen Studierenden war in den Jahren zuvor nach Deutschland geflüchtet. Sie bilden die größte Geflüchtetengruppe an den hiesigen Hochschulen. Schätzungsweise einer bzw. eine von zehn internationalen Studierenden hat Fluchterfahrung, Tendenz steigend - die Hochschulen sind insofern auch wichtige Motoren der Flüchtlingsintegration. Inwiefern diese Integrationsfunktion sich auch bis zum Einstieg in den deutschen Arbeitsmarkt erstreckt, ist bislang empirisch kaum untersucht (vgl. Schammann/Younso 2016: 45-47; Engel/Wolter 2017: 70-72).

3 Internationale Studierende sind ausländische Staatsangehörige, die die Schule im Ausland besucht und dort auch die Hochschulreife erworben haben. In der Hochschulstatistik werden internationale Studierende auch als „Bildungsausländerinnen“ bzw. „Bildungsausländer“ bezeichnet.

4 Für die Fachkommission der Bundesregierung zu den Rahmenbedingungen der Integrationsfähigkeit (2020: 149) zählen internationale Studierende zu den „neue[n] Zielgruppen“ für zukünftige Einbürgerungskampagnen.

5 Vgl. u. a. BMAS 2011: 55; BMI 2012: 54-56; BAMF 2014: 23; BMBF 2017: 30; Wissenschaftsrat 2016: 153-155; BDA/HRK/BDI 2014: 3-5; DAAD 2014a: 4; SVR-Forschungsbereich 2015: 29.

6 Wenn der derzeitige Trend von jährlich rund 50.000 internationalen Absolventinnen und Absolventen anhält, werden von 2021 bis 2025 bundesweit knapp eine viertel Million internationale Studierende einen Abschluss erwerben und der hiesigen Wirtschaft zur Verfügung stehen (DAAD/DZHW 2021; eigene Berechnung).

7 Bei den Studierenden aus Indien, dem zahlenmäßig zweitwichtigsten Herkunftsland internationaler Studierender in Deutschland, wuchs die Zahl im ersten Jahr der Pandemie von 24.868 auf 28.542. Viele waren möglicherweise bereits vor dem Erlass der Einreisebeschränkungen hierzulande in Sprach- und Vorbereitungskursen eingeschrieben (vgl. SVR-Forschungsbereich 2019: 17). Die Gesamtzahl der Studierenden aus Syrien, dem drittwichtigsten Herkunftsland, stieg derweilen von 15.948 auf 16.931. Einige westliche Herkunftsländer verzeichneten dagegen deutliche Rückgänge, z. B. Italien (-9\%), Spanien (-15\%) und die USA (-22 \%) (Statistisches Bundesamt 2021a; eigene Berechnung). Insgesamt ist derzeit schwer abzuschätzen, ob und gegebenenfalls wie sehr sich die Pandemie auf die zukünftige Studierendenmobilität auswirken wird. Einerseits bekunden viele der noch im Ausland lebenden internationalen Studieninteressierten, dass sie ihre Pläne für ein Studium in Deutschland nicht aufgegeben, sondern lediglich vertagt haben. Gleichzeitig geht knapp die Hälfte des Hochschulpersonals in den Akademischen Auslandsämtern von einem kurzfristigen Rückgang der aus dem Ausland kommenden Studienanfängerinnen und -anfänger aus (DAAD 2020b: 4; Jayadeva 2020: 6; Fintiba 2020: 5). 
Die integrationsbezogenen Aufgaben der Hochschulen werden durch die bundesfinanzierten Hochschulprogramme, die der Deutsche Akademische Austauschdienst in den letzten Jahren für Geflüchtete entwickelt hat, unterstützt. Die Erfahrungen der projektgeförderten Hochschulen erlauben nun einen ersten Einblick in die Erfahrungen Geflüchteter am Übergang vom Studium in den Beruf. Die vorliegende Expertise des wissenschaftlichen Stabs des SVR fasst diese Erfahrungen zusammen, vergleicht sie mit denen anderer internationaler Studierender und entwickelt Handlungsempfehlungen für die Hochschulpraxis und -politik.

Ein wichtiges Zugangstor zum Studium war und ist das seit 2016 laufende deutschlandweite DAAD-Studienvorbereitungsprogramm „Integration von Flüchtlingen ins Fachstudium“ (Integra), über das bereits in den ersten drei Förderjahren mehr als 26.000 Geflüchtete mit sprachlichen und fachlichen Vorbereitungskursen für ein Studium qualifiziert wurden.8 Die Zahl derjenigen, die bis heute über die mehr als 170 Integra-geförderten Hochschulprojekte den Weg in ein Studium gefunden haben, wird auf über 30.000 geschätzt (DAAD 2020a: 26). Aufbauend auf diesem Erfolg wurde für den Förderzeitraum 2020-2021 neben dem Studium und der Studienvorbereitung auch erstmals der spätere Berufseinstieg in den Blick genommen, nicht zuletzt, weil ein Teil der Geflüchteten bereits kurz vor dem Abschluss steht. In 71 der aktuell 130 Integra-geförderten Hochschulprojekte steht daher die Arbeitsmarktvorbereitung mit im Vordergrund. Diese Hochschulprojekte und die 4.233 Studierenden darin stellen die erste Säule der folgenden Analyse dar (Tab. 1).

Die zweite Säule bildet das vom DAAD im Jahr 2020 erstmalig ausgeschriebene „Programm zur Förderung der bildungsadäquaten Integration in den deutschen Arbeitsmarkt“ (PROFI), welches als Weiterbildungsprogramm für berufserfahrene und hochqualifizierte Flüchtlinge konzipiert ist. Im Förderzeitraum sollen die insgesamt 192 Teilnehmerinnen und Teilnehmer an 17 Hochschulen über kürzere und auf bestimmte Zielberufe und -branchen zugeschnittene Lernangebote für einen möglichst schnellen (Wieder-) Einstieg in den deutschen Arbeitsmarkt qualifiziert werden. Die Erfahrungen dieser für viele Hochschulen neuen Zielgruppe sind von hohem integrations- und arbeitsmarktpolitischen Interesse (vgl. Engel/Wolter 2017: 70-72).

Da sich der ursprünglich für April 2020 geplante Programmstart an den meisten Hochschulen aufgrund der bundesweiten Pandemielage deutlich verzögert hat und die geplanten Aktivitäten seither kontinuierlich an die sich ändernden Hygiene- und Kontaktregeln angepasst werden müssen, wurde die Projektlaufzeit um ein Jahr bis Dezember 2022 verlängert. Die im Folgenden präsentierten Forschungsergebnisse beziehen sich auf die erste Projektphase und beleuchten den Zeitraum von Mitte 2020 bis Anfang 2021.

TABELLE 1: HOCHSCHULPROJEKTE IN DEN PROGRAMMEN INTEGRA UND PROFI 2020-2022

\begin{tabular}{lrr} 
& PROFI \\
Anzahl geförderter Hochschulprojekte & $\mathbf{1 3 0}$ & $\mathbf{1 7}$ \\
& $(\mathrm{TN}: 7.467)$ & $(\mathrm{TN}: 192)$ \\
davon mit Schwerpunkt Arbeitsmarktvorbereitung & 71 & 17 \\
& $(\mathrm{TN}: 4.233)$ & (TN: 192) \\
\hline
\end{tabular}

TN = Teilnehmerinnen und Teilnehmer

Anmerkung: Die Daten geben den Stand Anfang 2021 wieder. Quelle: Eigene Auswertung der Daten aus Integra und PROFI

8 Neben Integra fördern eine Handvoll weiterer Programme den Studieneinstieg von Menschen mit Fluchterfahrung, u. a. das ebenfalls vom DAAD aufgesetzte nordrhein-westfälische Programm „NRWege ins Studium“ sowie das Programm „Welcome“, welches Studierendeninitiativen fördert, die Geflüchtete vor und während ihres Studiums unterstützen (DAAD 2020a: 13-14). 
Ziel der vorliegenden Begleitstudie ist es, die Erfahrungen aus den ,Reallaboren' der 88 untersuchten Integra- und PROFI-Projekte wissenschaftlich aufzuarbeiten und handlungsrelevante Erkenntnisse für die Förderung des Berufseinstiegs geflüchteter und anderer internationaler Zielgruppen abzuleiten. Aufbauend auf einer statistischen Auswertung ausgewählter Projektdaten stützt sich die explorative Studie insbesondere auf die vertiefende qualitative Analyse der schriftlich und mündlich festgehaltenen Erfahrungen von Projektverantwortlichen in Hochschulen, externen Partnerorganisationen sowie den Teilnehmenden selbst (Info-Box 1).

Das Erkenntnisinteresse der Studie gilt dabei nicht nur den Geflüchteten, sondern auch den knapp 300.000 anderen internationalen Studierenden, die nicht über Fluchterfahrung verfügen und von denen ein Großteil ebenfalls nach dem Studium in Deutschland bleiben und arbeiten möchte (SVR-Forschungsbereich 2017a: 16). Zwar können auch sie seit dem Jahr 2020 an den Integra- und PROFI-geförderten Bewerbungstrainings, Sprachkursen und Karriereberatungen teilnehmen, sie machen bislang aber nur einen kleineren Teil der jeweiligen Teilnehmerkreise aus (Tab. 8 im Anhang). Um die Erfahrungen dieser Gruppe dennoch systematisch mit zu berücksichtigen, werden die Studienergebnisse mit den einschlägigen Befunden aus der Bildungs- und Migrationsforschung komplementiert und kontrastiert, insbesondere der wachsenden Zahl an interdisziplinären Arbeiten zum Verbleib internationaler Studierender (u. a. SVR-Forschungsbereich 2015; 2017a; 2019; Dömling 2013; Arajärvi/Drubig 2014; Hanganu/Heß 2014; Übelmesser/Henn 2019).
Kap. 2 untersucht zunächst, vor welchen Einstiegshürden geflüchtete und andere internationale Zielgruppen beim Übergang vom Studium in den deutschen Arbeitsmarkt stehen. Die vielfältigen und im Zuge der Pandemie zumeist digital angebotenen Unterstützungsleistungen der Hochschulen werden in Kap. 3 ausgewertet. Ob und gegebenenfalls wie intensiv die Hochschulen bei ihrer Übergangsgestaltung mit Unternehmen, Kammern und anderen externen Partnerorganisationen kooperieren, steht im Zentrum von Kap. 4. Aufbauend auf den Forschungsergebnissen entwickelt das abschließende Kap. 5 Handlungsempfehlungen für Hochschulen und Politik. 


\section{INFOBOX 1: METHODISCHES VORGEHEN DER BEGLEITSTUDIE}

Um die bisherigen Erfahrungen aus den laufenden berufsvorbereitenden Vorhaben in den Programmen Integra und PROFI systematisch zu erfassen, stützt sich die vorliegende Begleitstudie zunächst auf eine statistische Auswertung der Sachberichte, die die Hochschulen im Frühjahr 2021, knapp ein Jahr nach Förderbeginn, eingereicht haben. Angesichts der überschaubaren Anzahl an untersuchten Universitäten, Fachhochschulen, Kunsthochschulen und anderen höheren Bildungseinrichtungen beschränkte sich die quantitative Auswertung auf deskriptive statistische Verfahren, wie etwa numerische und prozentuale Häufigkeitsverteilungen. Im Zentrum der Analyse standen 71 Integra- und 17 PROFI-geförderte Hochschulprojekte, deren Vorhaben einen starken Arbeitsmarktfokus aufweisen. Analog zu den bis dato öffentlich zugänglichen Integra-Sachberichten wurde auf multivariate Regressionen und andere inferenzstatistische Analysen verzichtet (vgl. DAAD 2017; 2018; 2020a).

Aufbauend auf den statistischen Daten liefern qualitative Analysen das Herzstück der vorliegenden Studie. Mithilfe einer inhaltsanalytischen Auswertung von mehr als 600 schriftlich ausformulierten Kommentaren und Kurzberichten des Hochschulpersonals kann die bislang kaum erforschte Berufseinstiegssituation von Geflüchteten an den hiesigen Hochschulen erstmals explorativ beschrieben werden. Ergänzt wird diese Gesamtschau durch eine vertiefende Analyse an neun Hochschulstandorten, die kriteriengeleitet anhand von hochschulischen (z. B. Studierendenzahl), projektspezifischen (z. B. PROFI-Förderung) und arbeitsmarktstrukturellen (z. B. regionale Arbeitslosenquote) Faktoren ausgewählt wurden. Zwischen Februar und April 2021 wurden 27 vertiefende Interviews mit Projektverantwortlichen in Hochschulen (9 Interviewte), externen Partnerorganisationen (8 Interviewte) sowie Geflüchteten (10 Interviewte) durchgeführt. Als Interviewpartnerinnen und -partner wurden zunächst die Verantwortlichen in den Hochschulen kontaktiert und um die Vermittlung zusätzlicher Interviewkontakte im projektbezogenen Netzwerk gebeten, z. B. einzelne Studierende oder die örtliche Agentur für Arbeit (sog. Gatekeeper-Sampling) (Kruse 2014). Die ca. 45-minütigen Interviews erfolgten leitfadengestützt per Videokonferenz. Der Interview-Leitfaden kann in Tab. 12 im Anhang eingesehen werden. Sämtliche Interviews wurden - nach schriftlicher Zustimmung der Interviewpartnerinnen und -partner - datenschutzkonform aufgezeichnet, vorübergehend gespeichert und anschließend vollständig transkribiert und ausgewertet.

Die qualitative Inhaltsanalyse der Interviews sowie der schriftlichen Antworten aus den Sachberichten stützte sich bei der Kategorienentwicklung zunächst auf bereits gewonnene Forschungserkenntnisse (sog. deduktive Kategorienentwicklung) und kombinierte diese schließlich mit neuen Ergebnissen, die in den Antworten zum Vorschein kamen (sog. induktive Kategorienentwicklung). Da keines der Projekte zum Zeitpunkt der Erhebung abgeschlossen war, konnten über die Inhaltsanalyse - verglichen mit rein quantitativen Erhebungen - viele zusätzliche Erkenntnisse gewonnen werden (vgl. Schreier 2014).

Wie in der qualitativer Forschung üblich, handelt es sich bei den schriftlich und mündlich befragten Personen nicht um eine statistisch repräsentative Stichprobe. Die Vielfalt der Stichprobe und die Detailauswertung der Antworten erlauben es aber, aus den geführten Interviews erste Schlüsse über die Erfahrungen von und mit Geflüchteten am Übergang vom Studium in den Arbeitsmarkt zu ziehen. Die Ergebnisse werden durch zahlreiche Interviewzitate veranschaulicht. Diese werden inhaltlich unverändert wiedergegeben, zugunsten der Lesbarkeit wurden sie lediglich sprachlich leicht geglättet. Aus den Zitaten wurden einzelne Sätze entfernt, die sich inhaltlich mit davor oder danach gemachten Äußerungen decken, oder sich auf andere Themen beziehen. Solche Auslassungen wurden durch [...] gekennzeichnet. Ebenfalls mit eckigen Klammern sind kurze redaktionelle Ergänzungen markiert, die helfen sollen, die Zitate besser zu verstehen. 


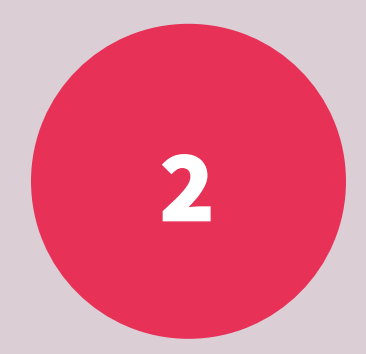

\section{Zentrale Hürden auf dem Weg in die Arbeitswelt}

2.1 Gleichermaßen betroffen: internationale Studierende mit und ohne Fluchterfahrung 
Der Verbleib internationaler Studierender im deutschen Arbeitsmarkt ist ein zunehmend wichtiger Bestandteil der Internationalisierungsbestrebungen von Hochschulen und (Hochschul-)Politik. ${ }^{9}$ Auch die Bildungsmigrantinnen und -migranten selbst betrachten ihr Studium nicht nur als eine Möglichkeit, den eigenen akademischen Horizont zu erweitern, sondern oft als ein ,Gesamtpaket Bildung + Arbeit' und somit als einen vielversprechenden legalen Zugang zum hiesigen Arbeitsmarkt (siehe u. a. SVR-Forschungsbereich 2017a; 2019). Allerdings ist der erfolgreiche Eintritt in den Arbeitsmarkt alles andere als ein Selbstläufer, trotz der mittlerweile großzügigen rechtlichen Bleibemöglichkeiten. ${ }^{10}$ Viele scheitern bei der Arbeitssuche zunächst an unzureichenden Deutschkenntnissen, fehlenden beruflichen Netzwerken und zu wenig Praxiserfahrung im Inland. ${ }^{11}$ Zudem sind viele Unternehmen nach wie vor zurückhaltend, wenn es um die Einstellung internationaler Absolventinnen und Absolventen geht, auch wenn diese ihren Abschluss in Deutschland erworben haben - dies gilt zumal, wenn sie einen großen Teil ihrer akademischen und beruflichen Vorerfahrung im Ausland gesammelt haben. Insbesondere kleine und mittelständische Betriebe befürchten einen hohen Verwaltungs- und Integrationsaufwand und wissen mit den ausländischen Werdegängen der
Bleibewilligen nur wenig anzufangen (Arajärvi/ Drubig 2014: 8; Hanganu/Heß 2014: 150; Übelmesser/Henn 2019: 18-24).

Ob und inwiefern diese Einstiegshürden auch für die mittlerweile rund 30.000 Geflüchteten gelten, die hierzulande studieren, ist bislang ungeklärt (SVR-Forschungsbereich 2019: 32-35). Die DAAD-Programme Integra und PROFI liefern hierzu erste Erkenntnisse. Beide sprechen mit ihren Programmschwerpunkten Flucht und Arbeitsmarkintegration neue Zielgruppen an, die zusätzlich zu ihrer individuellen Fluchterfahrung häufig älter und mitunter auch berufserfahrener sind als ihre Kommilitoninnen und Kommilitonen (Tab. 2). Inwiefern diese neuen Zielgruppen beim Übergang von der Hochschule in den deutschen Arbeitsmarkt auf neue bzw. andersartige Hürden treffen, zeigen die folgenden Erkenntnisse für geflüchtete Studierende im Integra-Programm (s. Kap. 2.1) sowie für die älteren und berufserfahreneren Geflüchteten, die über PROFI an einer hochschulischen Weiterbildung teilnehmen (s. Kap. 2.2).

TABELLE 2: ALTERSSTRUKTUR AUSGEWÄHLTER STUDIERENDENGRUPPEN AN DEUTSCHEN HOCHSCHULEN

\begin{tabular}{lrrrr} 
& $\begin{array}{r}\text { inländische } \\
\text { Alter }\end{array}$ & $\begin{array}{r}\text { internationale } \\
\text { Studierende (2016) }\end{array}$ & $\begin{array}{r}\text { Teilnehmende } \\
\text { Studierende (2016) }\end{array}$ & $\begin{array}{l}\text { Integra (2021) } \\
\text { PROFI (2021) }\end{array}$ \\
bis 25 Jahre & $67 \%$ & $53 \%$ & $30 \%$ & $3 \%$ \\
\hline 26 bis 30 Jahre & $23 \%$ & $26 \%$ & $34 \%$ & $21 \%$ \\
\hline $\begin{array}{l}\text { älter als 30 } \\
\text { Jahre }\end{array}$ & $10 \%$ & $21 \%$ & $36 \%$ & $76 \%$ \\
\hline
\end{tabular}

Anmerkung: Zu den inländischen Studierenden zählen alle, die über eine deutsche Hochschulzugangsberechtigung verfügen, unabhängig von ihrer Staatsangehörigkeit. Die Daten zu inländischen und internationalen Studierenden beziehen sich auf das Jahr 2016, das aktuellste Jahr, für das repräsentative Altersangaben vorliegen.

Quellen: Apolinarski/Brandt 2018: 36; Middendorff et al. 2017: 24; eigene Auswertung der Integra- und PROFI-Daten

9 Siehe u. a. BMAS 2011: 55; BMI 2012: 54-56; BAMF 2014: 23; BMBF 2017: 30; Wissenschaftsrat 2016: 153-155; BDA/HRK/BDI 2014: 3-5; DAAD 2014a: 4; SVR-Forschungsbereich 2015: 26-31; CSND 2018: 10.

10 Nach ihrem Abschluss können internationale Studierende zunächst bis zu 18 Monate in Deutschland bleiben, um eine Arbeitsstelle zu suchen. In dieser Suchphase können sie uneingeschränkt arbeiten (§20 Abs. 3 Aufenthaltsgesetz). Ist ihre Suche erfolgreich, können sie bereits nach zwei Jahren Arbeit in Deutschland in einen Daueraufenthalt wechseln (§ 18c Abs. 1 Aufenthaltsgesetz).

11 Erschwerend kommt hinzu, dass 49 Prozent der internationalen Studierenden ihr Bachelorstudium und 26 Prozent ihr Masterstudium ohne Abschluss beenden. Sie sind damit weniger erfolgreich als ihre deutschen Mitstudierenden (27 und 17\%) (Heublein/Richter/Schmelzer 2020: 4-11). 


\subsection{Gleichermaßen betroffen: internationale Studierende mit und ohne Fluchterfahrung}

Anfang 2021 nahmen bundesweit 4.233 Studierende an den studien- und berufsvorbereitenden Angeboten teil, die an 71 Hochschulen mithilfe des Integra-Programms entwickelt wurden. Über die Hälfte der Studierenden war in den Vorjahren nach Deutschland geflüchtet, ihre Erfahrungen können somit Aufschluss darüber geben, inwiefern eine Flucht auch dann noch den Berufseinstieg erschwert, wenn auf sie ein anerkanntes Studium folgt. ${ }^{12}$

Die zentralen Hürden, vor denen geflüchtete Studierende beim Berufseinstieg in Deutschland stehen, werden im Folgenden zusammengefasst und mit denen ihrer internationalen Mitstudierenden verglichen. ${ }^{13} \mathrm{Da}$ bereits zu Studienbeginn wichtige Weichen für den späteren Überwerden (können), wird die Studieneingangsphase punktuell mitbeleuchtet.

\section{DEUTSCHKENNTNISSE ALS GRUND- LEGENDE EINGANGSVORAUSSETZUNG FÜR STUDIUM UND BERUF}

Zwar wünschen sich viele Geflüchtete - ähnlich wie die hiesige Politik und Wirtschaft - einen möglichst raschen Einstieg in die Studien- und Arbeitswelt (Brücker et al. 2016: 119; OECD 2017: 44), die Praxis im Integra-Programm veranschaulicht allerdings, dass der Übergang in ein Studium sowie vom Studium in den Beruf deutlich schwerer fällt, wenn beim Hochschulzugang die sprachliche und fachsprachliche Vorbereitung verkürzt oder gar übersprungen wird.

Bevor Geflüchtete voll in ihr Wunschstudium einsteigen können, müssen sie zunächst - wie alle anderen internationalen Studieninteressierten - fortgeschrittene Deutschkenntnisse der Stufe B2 oder $\mathrm{C}^{14}$ nachweisen. Zur Vorbereitung steht ihnen eine Vielzahl an Sprachkursen zur Verfügung, die von Bund, Ländern, Kommunen und nichtstaatlichen Organisationen finanziert und zu unterschiedlichen Kosten angeboten werden. Außerhalb der Hochschulen verhelfen die vom Bundesamt für Migration und Flüchtlinge geförderten Integrationskurse zu leicht fortgeschrittenen Deutschkennnissen, allerdings i. d. R. nur bis zum Niveau B1. Einen schnellen Zugang zum Integrationskurs erhalten zudem meist nur Personen, deren Asylantrag bewilligt wurde und die eine Aufenthaltserlaubnis erhalten haben. Sie haben einen rechtlichen Anspruch darauf und sind oft sogar zur Teilnahme verpflichtet (§ 44a Abs. 1 Aufenthaltsgesetz). Anders ist es bei Asylsuchenden mit Aufenthaltsgestattung, d. h. Personen, bei denen das Asylverfahren noch läuft, sowie auch Personen, deren Antrag abgelehnt wurde, die aber noch in Deutschland leben, weil eine Ausreise (noch) nicht möglich ist (sog. Geduldete): Sie müssen sich je nach Wohnort und der dortigen Verfügbarkeit mitunter länger gedulden (Tab. 3).

Um diese und andere Angebotslücken zu schließen, haben viele der Hochschulen im Integra-Programm in den vergangenen Jahren verstärkt studienvorbereitende Deutschkurse und zum Teil auch Englischkurse entwickelt (DAAD 2018: 25-26; SVR-Forschungsbereich 2019: 33). Anders als beim Integrationskurs ist der Zugang zur Hochschule nicht vom Aufenthaltstitel abhängig, sondern davon, ob die sprachlichen und fachlichen Voraussetzungen erfüllt sind (Tab. 3). Oft waren und sind diese Sprachkurse studienvorbereitend, d. h. sie sind in die

1255 Prozent der insgesamt 4.233 Studierenden waren nach Deutschland geflüchtet. Für knapp ein Drittel lagen keine Informationen zum Fluchthintergrund vor (Tab. 8 im Anhang). Rechnet man diesen Teil heraus, betrug der Anteil der geflüchteten Studierenden im IntegraProgramm Anfang 202178 Prozent. Nicht alle der durch Integra geförderten Hochschulen bereiten Studierende im Rahmen der Projekte auf den Berufseinstieg vor. Neben den hier betrachteten 71 Hochschulen erhielten 59 weitere Bildungseinrichtungen Fördermittel vom DAAD, zumeist für studienvorbereitende Maßnahmen. Diese waren nicht Teil der Analyse.

13 Die Befunde zu internationalen Studierenden ohne Fluchterfahrung entstammen einschlägigen Studien der Hochschul- und Migrationsforschung.

14 In englischsprachigen Studiengängen ist ein entsprechendes Sprachniveau vonnöten. Sprecherinnen und Sprecher mit Niveaustufe C1 nach dem Gemeinsamen Europäischen Referenzrahmen für Sprachen können die deutsche bzw. die englische Sprache in Studium, Beruf und Alltag ohne größere Schwierigkeiten anwenden. Sie können komplexe Sachtexte ohne Probleme erfassen und anspruchsvolle Sachverhalte erklären, ohne öfter nach Worten suchen zu müssen. 
entsprechenden Hinführungsprogramme integriert und geben den Teilnehmenden einen Einblick in ihr zukünftiges Studienfach. Anders als viele der internationalen Studieninteressierten, die eigens für das Studium und mitunter auch für die sprachliche und fachliche Studienvorbereitung nach Deutschland zuwandern, verfügen Geflüchtete oft über gute alltagssprachliche Deutschkenntnisse. ${ }^{15}$ Diese ergeben sich sowohl aus ihrem i. d. R. längeren Voraufenthalt und dem entsprechenden Zugang zu Integrationskursen als auch aus dem Wunsch und der empfundenen Notwendigkeit, Deutsch zu lernen, um am sozialen Leben hierzulande zu partizipieren (vgl. SVR-Forschungsbereich 2016a). Beim Erlernen von Deutsch als Wissenschaftssprache stehen beide Zuwanderergruppen jedoch vor sehr ähnlichen Hürden. Selbst diejenigen, die fließend Deutsch sprechen, tun sich oft schwer mit dem Fachjargon, der das Kursgeschehen bestimmt. Beim Verfassen wissenschaftlicher Texte benötigen sie oft zusätzliche Unterstützung, um ihre Angst vor dem Schreiben in der Fremdsprache abzubauen, sich neue Schreibtechniken anzueignen und zu lernen, wie sie ihre eigenen Texte überarbeiten können (vgl. Brandl 2007: 158-161; Voigt 2011: 38-39).

Doch nicht nur im Studium sind fachsprachliche Kenntnisse mit erfolgsentscheidend, auch beim späteren Berufseinstieg bestimmen sie maßgeblich den individuellen Werdegang (siehe u. a. OECD 2017: 34-39). Das befragte Personal und die Studierenden im Integra-Programm betonen dies nahezu einstimmig und weisen darüber hinaus darauf hin, dass ein Teil der Betriebe überzogene und unrealistische sprachliche Anforderungen an internationale Fachkräfte stellt, und dies nicht nur hinsichtlich der Fachsprache: Mancherorts berichten die Befragten, dass selbst Kenntnisse des regionalen Dialekts als Einstellungskriterium herangezogen werden. Der daraus entstehende Erwartungsdruck kann Unsicherheiten schüren, wie das folgende Zitat beispielhaft veranschaulicht:
Wenn ich z. B. eine Bewerbung schicke und [die Personalabteilung] sieht, dass ich erst seit 2015 hier bin und Deutsch nicht meine Muttersprache ist, dann denken sie, dass ich nicht so gut die Sprache spreche und vielleicht neigen sie dazu, eher einen Muttersprachler oder Muttersprachlerin zu nehmen, was auch ein bisschen verständlich ist, weil Unternehmen, also, die meisten Unternehmen hier denken an erster Stelle an den Gewinn. [...] Die Sprache spielt immer eine Rolle, also, die meisten können schon gut Deutsch, aber können das nicht so direkt zeigen, also, nicht jeder kann so direkt in einem dreißigminütigen Gespräch zeigen, dass man gut Deutsch spricht und dann wird das als Schwäche angesehen.

(Afghanischer Bachelorstudent an einer Universität, Integra-Programm)

Insgesamt zeigen die Befunde aus den IntegraProjekten und der Forschungsliteratur, dass fach- und wissenschaftssprachliche Kenntnisse eine große Herausforderung darstellen. Unabhängig vom Fluchthintergrund treffen die beschriebenen Hürden und Sorgen somit auf einen großen Teil der internationalen Studierenden zu, die Deutsch (noch) nicht auf einem fortgeschrittenen fachsprachlichen Niveau beherrschen.

\section{MANGELNDE SYSTEMKENNTNIS UND FINANZIELLE ENGPÄSSE ERSCHWEREN WERTVOLLE PRAXISERFAHRUNG}

Internationale Studierende, die frühzeitig im Studium fachnah Praxiserfahrungen sammeln, haben später bessere Chancen auf einen erfolgreichen Berufseinstieg (SVR-Forschungsbereich 2017a: 9; Kratz/Reimer 2013: 2-5). Die Forschung zu internationalen Studierenden zeigt allerdings, dass die Bleibewilligen unter ihnen auf ihrer Suche nach einem Praktikumsplatz oder einer studentischen Hilfskraftstelle neben sprachlichen Hürden viele weitere Hindernisse überwinden müssen. Nur wenige sind mit den Gepflogenheiten auf dem deutschen Arbeitsmarkt vertraut. Sie

15 Dies verdeutlichen die Deutschkenntnisse von Studienbewerberinnen und -bewerbern aus zahlenmäßig wichtigen Asylherkunftsländern, wie etwa Syrien. Hier haben 35 Prozent bereits vor Studienbeginn die Stufe C1 erreicht. Zum Vergleich: Studierwillige aus Indien verfügen lediglich zu 15 Prozent über entsprechende Deutschkompetenzen (DAAD/DZHW 2021). Dies dürfte u. a. damit zusammenhängen, dass ein großer Teil von ihnen englischsprachige Studienangebote bevorzugt. 
TABELLE 3: ZUGANG ZU STUDIENVORBEREITUNG, STUDIUM UND ARBEITSMARKT FÜR GEFLÜCHTETE

\begin{tabular}{|c|c|c|c|}
\hline & \multicolumn{3}{|c|}{ geflüchtete Studieninteressierte mit } \\
\hline & $\begin{array}{l}\text { Aufenthalts- } \\
\text { erlaubnis }\end{array}$ & $\begin{array}{l}\text { Aufenthaltsgestattung } \\
\text { (während des Asylverfahrens) }\end{array}$ & $\begin{array}{l}\text { Aufenthaltsduldung } \\
\text { (Aussetzung der Ausweisung) }\end{array}$ \\
\hline $\begin{array}{l}\text { Zugang zur Hochschule } \\
\text { Grundvoraussetzung: } \\
\text { · Hochschulzugangs- } \\
\text { berechtigung } \\
\text { · Sprachkenntnisse }\end{array}$ & ja & ja & ja \\
\hline Integrationskurs & ja & $\begin{array}{l}\text { ja, wenn } \\
\text { · aus Eritrea, Somalia oder } \\
\text { Syrien (wegen der guten } \\
\text { Chance auf Anerkennung) } \\
\text { ja, nach drei Monaten mit Auf- } \\
\text { enthaltsgestattung, wenn } \\
\text { · aus anderen Ländern und } \\
\text { · Einreise vor dem 01.08.2019 } \\
\text { und } \\
\text { •,arbeitsmarktnah“ } \\
\text { (s. Anm.) } \\
\text { grundsätzlich ausgeschlossen: } \\
\text { · Personen mit Aufenthalts- } \\
\text { gestattung aus sicheren } \\
\text { Herkunftsstaaten }\end{array}$ & $\begin{array}{l}\text { nein, außer bei } \\
\text { · Duldung zur Ausbildung, } \\
\text { Beschäftigung sowie wei- } \\
\text { terer „dringender humani- } \\
\text { tärer oder persönlicher } \\
\text { Gründe“ ( } \$ 60 \text { a Abs. } 2 \\
\text { Aufenthaltsgesetz) } \\
\text { grundsätzlich ausgeschlossen: } \\
\text { · Personen mit Duldung aus } \\
\text { anderen Gründen } \\
\text { · Personen mit Duldung aus } \\
\text { sicheren Herkunftsstaaten }\end{array}$ \\
\hline $\begin{array}{l}\text { Zugang zu BAföG } \\
\text { Grundvoraussetzung: } \\
\text { · Förderbedarf }\end{array}$ & ja & $\begin{array}{l}\text { nein, aber Anspruch auf monat- } \\
\text { liche Zahlungen nach dem } \\
\text { Asylbewerberleistungsgesetz }\end{array}$ & ja, nach 15 Monaten \\
\hline $\begin{array}{l}\text { Zugang zu } \\
\text { (Pflicht-)Praktika } \\
\text { (bis zu } 3 \text { Monate) }\end{array}$ & ja & $\begin{array}{l}\text { ja, nach drei Monaten, wenn } \\
\text { · Ausländerbehörde zustimmt } \\
\text { grundsätzlich ausgeschlossen: } \\
\text { · Personen mit Aufenthalts- } \\
\text { gestattung aus sicheren } \\
\text { Herkunftsstaaten }\end{array}$ & $\begin{array}{l}\text { ja, wenn } \\
\text { Ausländerbehörde zustimmt } \\
\text { i. d. R. ausgeschlossen: } \\
\text { · Personen mit Duldung aus } \\
\text { sicheren Herkunftsstaaten }\end{array}$ \\
\hline $\begin{array}{l}\text { Zugang zum } \\
\text { Arbeitsmarkt }\end{array}$ & ja & $\begin{array}{l}\text { ja, nach drei Monaten, wenn } \\
\text { · Ausländerbehörde zustimmt } \\
\text { · Agentur für Arbeit zustimmt } \\
\text { grundsätzlich ausgeschlossen: } \\
\text { · Personen mit Aufenthalts- } \\
\text { gestattung aus sicheren } \\
\text { Herkunftsstaaten }\end{array}$ & $\begin{array}{l}\text { ja, nach sechs Monaten mit } \\
\text { Duldung, wenn } \\
\text { · Ausländerbehörde und } \\
\text { Agentur für Arbeit zustimmen } \\
\text { i. d. R. ausgeschlossen: } \\
\text { · Personen mit Duldung aus } \\
\text { sicheren Herkunftsstaaten }\end{array}$ \\
\hline
\end{tabular}

Anmerkung: Als ,arbeitsmarktnah“ werden insbesondere Personen verstanden, die bei der Bundesagentur für Arbeit als ausbildungs- oder arbeitssuchend bzw. arbeitslos, beschäftigt oder in Ausbildung(svorbereitung) gemeldet sind. Nach Maßgabe des Asylgesetzes sind Albanien, Bosnien und Herzegowina, Ghana, Kosovo, Mazedonien, Montenegro, Senegal, Serbien sowie alle EU-Mitgliedstaaten derzeit als sichere Herkunftsstaaten eingestuft.

Quelle: eigene Zusammenstellung der einschlägigen asyl- und aufenthaltsrechtlichen Bestimmungen $(\S 25,44,60$ a Aufenthaltsgesetz, $\S 55,61$ Asylgesetz, § 2 Asylbewerberleistungsgesetz) in Anlehnung an BAMF (2016). 
wissen zunächst nicht, welche praktischen Fertigkeiten und Vorerfahrungen Unternehmen erwarten oder wie Bewerbungsverfahren hierzulande ablaufen. Zudem fehlt es vielen an sozialen und beruflichen Netzwerkkontakten, die ihnen beim Einstieg in die hiesige Berufswelt helfen können. Die Folge: Ohne entsprechende Praxiseinblicke und ein Unterstützungsnetzwerk stehen die Bleibewilligen bei der Arbeitssuche vor besonders hohen Hürden (Klabunde 2014: 29; Meschter et al. 2015: 10; SVR-Forschungsbereich 2017a: 19-20).

Die Erfahrungen der geflüchteten Studierenden im Integra-Programm bestätigen dies. Trotz ihres oft längeren Deutschlandaufenthalts stehen auch sie vor ähnlichen Herausforderungen wie ihre internationalen Mitstudierenden. Auch sie stammen häufig aus Regionen, in denen Berufseinstieg und Bewerbungsverfahren deutlich weniger formalisiert sind als in Deutschland. Und auch ihnen fehlt es oft an grundlegendem Wissen über den deutschen Arbeitsmarkt. Nur wenigen ist beispielsweise zu Studienbeginn bewusst, dass für sie auch kleine und mittelständische Betriebe jenseits der bekannten, weltweit agierenden Unternehmen für ein Praktikum oder später für eine Vollbeschäftigung in Frage kommen können. Sie benötigen zunächst grundlegende Informationen über die Vorzüge von Initiativbewerbungen und die zentrale Bedeutung von studienbegleitenden Praxiserfahrungen in Form von studentischen Hilfskraftstellen, Praktika und ehrenamtlichen Tätigkeiten (vgl. SVR-Forschungsbereich 2017a: 30; 2019: 28-29). Folglich betont das befragte Hochschulpersonal einen zusätzlichen Bedarf an zielgerichteter Praktikumsplanung und frühzeitiger Berufsorientierung.

Auch finanzielle Engpässe erschweren das Sammeln von Praxiserfahrung: Für einen Großteil der Geflüchteten ist ein Studium in Deutschland mit einer hohen zeitlichen und finanziellen Investition verbunden. Ein schneller Studienabschluss steht daher bei vielen Integra-Teilnehmenden im Vordergrund. Sie möchten so bald wie möglich in eine qualifizierte Beschäftigung und die finanzielle Unabhängigkeit eintreten. Dieser wirtschaftliche Handlungsdruck ist bei Geflüchteten ebenso hoch wie bei vielen anderen internationalen Studierenden, zumal nur wenige auf familiäre Finanzzuschüsse hoffen können und ohne fremde Hilfe kaum in der Lage sind, die komplizierten $\mathrm{Zu}$ gangsregeln zum BAföG und - im Falle derjenigen, deren Asylverfahren deutlich länger dauert - zum Asylbewerberleistungsgesetz zu überblicken. ${ }^{16}$ Für diejenigen, die nicht den BAföG-Höchstsatz erhalten, sind unbezahlte oder geringfügig vergütete Praktika deutlich schwerer realisierbar. Die folgenden zwei Zitate verweisen auf diesen Druck und die Versäumnisse, die während des Studiums daraus entstehen können:

Das Problem ist finanziell, also, wenn ich jetzt ein Praktikum machen werde, kann ich meine Arbeit [= fachfremder Nebenjob] nicht weitermachen und ich muss $z$. B. Vollzeit ein Praktikum machen und da kann mich gar keiner finanzieren. Das ist ein sehr großes Problem für mich gerade.

(Afghanischer Bachelorstudent an einer Universität, Integra-Programm)
Ich habe gesagt, ,Okay, ich fokussiere mich einfach auf mein Studium, mache das so schnell wie möglich fertig und dann kann ich nachher arbeiten. 'Aber das war ein Fehler, das muss ich schon zugeben. (Syrischer Masterstudent an einer Universität, Integra-Programm)

16 Internationale Studierende ohne Fluchterfahrung haben durchschnittlich 776 Euro im Monat zur Verfügung. Knapp die Hälfte ihres Einkommens stammt von der Familie. Zudem müssen die Studierenden ein Drittel durch Kellnern, Aushilfsjobs und andere Nebentätigkeiten selbst verdienen, da eine BAföGFörderung für die meisten rechtlich ausgeschlossen ist (Apolinarski/Brandt 2018: 42). Anders bei Geflüchteten im Integra-Programm: 78 Prozent erhalten nach asylrechtlicher Anerkennung oder einer mindestens 15-monatigen Duldung BAföG-Zahlungen von durchschnittlich knapp über 700 Euro pro Monat (DAAD 2020a: 53; Statistisches Bundesamt 2021b). Diese Unterstützung ist je nach Aufenthaltsstatus leichter oder schwieriger zugänglich (Tab. 3) und wird jährlich neu berechnet. Hierbei kann die Vorbildung im Ausland zum Hindernis werden. Für Geflüchtete mit ausländischer Studienerfahrung oder einem Abschluss sind Fachwechsel oder eine Einschreibung in einem Bachelor-Studium nur in Ausnahmefällen vorgesehen, denn genau wie deutsche Empfängerinnen und Empfänger sollen sie dort weiterstudieren, wo sie vor ihrer Antragsstellung aufgehört haben. Dass ihre Vorbildung im Ausland mitunter keine guten Arbeitsmarktaussichten verspricht und ein Fach- oder Studienwechsel zum Teil zielführender wäre, wird bislang nur in Ausnahmefällen berücksichtigt. Angesichts dieser Zugangshürden ist eine intensive individuelle Beratung und Betreuung vonnöten (vgl. Knuth 2020: 367; Gödersmann 2021: 53-54). Dies gilt nicht zuletzt auch für Personen mit Aufenthaltsgestattung, deren Asylverfahren länger dauert (Tab. 3). Sie haben bis zur Erteilung einer Aufenthaltserlaubnis keinen BAföG-Zugang und erhalten Mittel nach dem Asylbewerberleistungsgesetz (Bridge Netzwerk 2021: 39-41). 


\section{ZURÜCKHALTENDE UNTERNEHMEN UND DISKRIMINIERUNGSERFAHRUNGEN}

Die Zurückhaltung vieler Unternehmen bei der Einstellung von internationalen Bewerberinnen und Bewerbern stellt ein weiteres Hemmnis für internationale Studierende mit und ohne Fluchterfahrung dar (vgl. SVR-Forschungsbereich 2019: 29; Lancee 2019: 12-14; Ekert et al. 2014: 59). Den Schilderungen der Studierenden und Programmverantwortlichen in den Integra-Projekten zufolge reichen die Gründe der arbeitgeberseitigen Zurückhaltung von aufenthaltsrechtlichen Bedenken bis hin zu Vorurteilen gegenüber bestimmten Herkunftsgruppen. In letzterer Hinsicht unterscheiden sich diese Erfahrungen kaum von denen anderer internationaler Studierender.

Das befragte Hochschulpersonal berichtet, dass es den Unternehmen vor Ort häufig an kulturell und sprachlich sensiblen Auswahlverfahren mangelt - zum Nachteil der Integra-Studierenden. Geflüchtete müssen demnach häufig mehr Bewerbungen schreiben als deutsche Studierende, um eine Einladung zum Auswahlgespräch zu erhalten, auch wenn sie die fachlichen Anforderungen erfüllen. Schon kleinere formale Fehler können dazu führen, dass Betriebe die sprachlichen und fachlichen Kompetenzen der internationalen Bewerberinnen und Bewerber in Frage stellen:

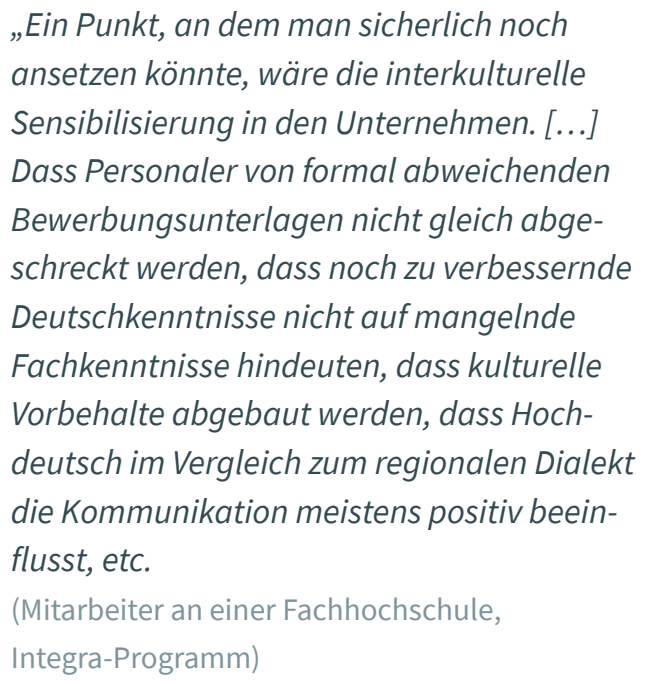

Diese Berichte decken sich mit den einschlägigen Befunden aus der Arbeitsmarktforschung, die zeigen, dass Menschen mit Migrationshintergrund in Einstellungsverfahren oft mehr Bewerbungen schreiben müssen und höhere Erwartungen zu erfüllen haben als Personen ohne Migrationshintergrund (u. a. Lancee 2019; SVR-Forschungsbereich 2014; SVR 2021: 86). Zudem erklärt das Hochschulpersonal, dass viele kleine und mittelständische Betriebe langfristige Arbeitsbeziehungen bevorzugen und mitunter befürchten, dass internationale Fachkräfte (auch solche mit hiesigem Hochschulabschluss) nicht lange im Unternehmen bleiben. Folglich stellen viele trotz des wachsenden Fachkräftebedarfs nach wie vor lieber den regional verwurzelten Nachwuchs ein. Dieser Pool schrumpft allerdings vielerorts und wird in den kommenden Jahren Rückgänge von bis zu 25 Prozent verzeichnen (BBSR 2018: 12).

Kleinere Personalabteilungen oder Betriebe ohne eigene Personalabteilung scheuen derweilen den bürokratischen Aufwand, den sie mit der Einstellung von internationalen Fachkräften verbinden, nicht zuletzt, weil sie die diesbezüglichen aufenthalts- und arbeitsrechtlichen Bestimmungen nicht oder nur sehr lückenhaft kennen. Einige hegen zudem den Verdacht, dass internationale Fachkräfte Betriebsgeheimnisse weitergeben könnten, und schließen eine Anstellung deshalb aus (vgl. Dömling 2013: 6; Arajärvi/Drubig 2014: 61-62; SVR-Forschungsbereich 2019: 30).

Vereinzelt bemerken das Hochschulpersonal und die Studierenden bei den Betrieben entsprechende Vorurteile und Diskriminierungstendenzen in Bezug auf den kulturellen Hintergrund, die Herkunft oder die Religion einzelner Zuwanderergruppen. Ein Teil der interviewten Integra-Studierenden fühlt sich bei der Arbeitssuche oder am Arbeitsplatz auf die eigene Zuwanderungsgeschichte reduziert und mit Vorurteilen konfrontiert. Dies beeinträchtigt nicht nur das persönliche Wohlbefinden, sondern kann auch beruflich zurückwerfen:

Das habe ich [bei meiner damaligen Arbeit] im Krankenhaus erlebt. Das hat sich damals ein bisschen negativ ausgewirkt. Und ich glaube, das war ein Grund, warum ich die 


\author{
Arbeit verlassen habe. Das Umfeld hat mir \\ gar nicht gefallen. Genau, das war wegen \\ meiner sprachlichen Schwäche, meine \\ Sprachbarriere war der Hauptgrund, warum \\ die anderen Krankenschwestern mich ein \\ bisschen schlecht behandelt haben, und \\ deshalb habe ich mich nicht wohl gefühlt. \\ Das war die Rassismuserfahrung, sozusa- \\ gen, die ich erlebt habe. \\ (Syrische Bachelorstudentin an einer \\ Universität, Integra-Programm)
}

Auch internationale Studierende ohne Fluchterfahrung berichten punktuell von Diskriminierungserfahrungen. Knapp ein Fünftel fühlt sich in Deutschland aufgrund der Hautfarbe, eines Akzents, der Religion oder aus anderen Gründen diskriminiert. Verbale oder gar physische Angriffe kommen eher selten vor (DAAD 2014b: 100-113). Die Geflüchteten im IntegraProgramm berichten ebenfalls kaum von derartigen Anfeindungen am Arbeitsplatz, betonen aber gleichzeitig, dass diese durchaus in der Öffentlichkeit auftreten können:

\section{Und hier in [Name der Stadt] ist Diskriminie- rung etwas hoch eigentlich. Hat sich verbes- sert, also, als ich hier angekommen bin 2016 da war es richtig schlimm, also, da wurden wir z. B. auf der Straße mit Mittelfinger be- gegnet. Auch Kinder, also geflüchtete Kin- der, wurden auch so begegnet. Einmal hat jemand versucht, mich zu schlagen auf der Straße. Es gab gute und schlechte Beispiele [...], z. B. in meiner Arbeit in der Gastrono- mie, da habe ich Diskriminierungen erlebt. (Iranischer Bachelorstudent an einer Universität, Integra-Programm)}

Über das Ausmaß dieser Ressentiments herrscht beim befragten Hochschulpersonal und bei den Studierenden jeweils Uneinigkeit. Ob und in welchem Maße prominent vertretene öffentliche Meinungen zum Thema Migration das Integrationsklima vor Ort beeinflussen - und damit auch zukünftige Bemühungen der Hochschulen um Internationalisierung -, kann mit der vorliegenden Begleitstudie nicht abschließend beantwortet werden. Die wechselseitige Beeinflussung zwischen hochschulischer Internationalisierungsarbeit und dem lokalen sowie dem überregionalen und medialen Integrationsklima erscheint aber als ein lohnenswertes Betätigungsfeld für zukünftige Forschungsarbeiten.

\section{UNTERBROCHENE BILDUNGSBIOGRAFIE ERSCHWERT HOCHSCHULZUGANG UND SPÄTEREN BERUFSEINSTIEG}

Die oben beschriebenen sprachlichen, arbeitskulturellen und strukturellen Hürden müssen vor dem Hintergrund der unterschiedlichen Bildungsbiografien von Studierenden mit und ohne Fluchterfahrung verstanden werden: Diejenigen, die speziell zum Studieren nach Deutschland kommen, haben zu 60 Prozent bereits ein Studium im Ausland abgeschlossen und möchten nun einen weiteren Abschluss erwerben (Apolinarski/Brandt 2018: 16). Auch ein großer Teil der Geflüchteten im Integra-Programm hat im Vorfeld im Ausland studiert, musste die Ausbildung aber fluchtbedingt unterbrechen. Der Wunsch nach einem anerkannten Bildungsabschluss in Deutschland, der auf den bereits erworbenen akademischen Qualifikationen aufbaut, spielt für sie somit eine besonders große Rolle, wie das folgende Zitat verdeutlicht:

Ich habe eigentlich schon in Syrien fast ein Studium absolviert, also, das war Physik. Ich habe schon sieben Semester studiert, aber leider habe ich das achte Semester nicht geschafft, denn ich konnte nicht. Weil bei uns, wenn man [das Studium] absolviert, dann darf man nicht raus aus Syrien. Dann kriegt man keinen Pass. [...] Wenn ich die Möglichkeit hätte, dann würde ich wahrscheinlich auch einen Master oder noch zwei Semester machen an einer anderen Uni, sodass ich mein altes Studium auch weitermache.

\section{(Syrischer Bachelorstudent an einer}

Fachhochschule, Integra-Programm)

Trotz dieser und anderer Vorerfahrungen gelingt der Einstieg ins Studium nicht immer. Von den insgesamt 6.844 Bewerbungen auf Integrageförderte Studienplätze im Wintersemester 
TABELLE 4: BEWERBUNGEN FÜR DIE TEILNAHME IN DEN INTEGRA- UND PROFI-PROJEKTEN

$\begin{array}{lrr} & \text { PROFI } \\ \text { Bewerbungen insgesamt } & \mathbf{5 1 9} \\ \text { davon } & \mathbf{6 . 8 4 4} & 317 \\ \quad \text { abgelehnt: } & 2.199 & 192 \\ \quad \text { angenommen (eingeschrieben) } & 4.233 & 10 \\ \quad \text { angenommen (nicht eingeschrieben) } & 412 & \end{array}$

Quelle: Eigene Auswertung der Daten aus Integra und PROF

2020/2021 wurden 2.199 abgelehnt; das entspricht knapp einem Drittel (Tab. 4). ${ }^{17} 74$ Prozent der Hochschulen gaben an, dass sie Studieninteressierte wegen fachlicher Lücken nicht ins Wunschstudium bzw. den entsprechenden Vorbereitungskurs aufnehmen konnten. An 44 Prozent der Hochschulen waren unvollständige Unterlagen mitausschlaggebend für eine $\mathrm{Ab}$ lehnung (Tab. $9 \mathrm{im}$ Anhang).

Zudem stellen die asyl- und aufenthaltsrechtlichen Bestimmungen eine Hürde dar. Zwar verhindern diese nicht zwangsläufig eine Einschreibung an einer Hochschule, da der Aufenthaltsstatus in allen Ländern seit 2014 kein hochschulrechtliches Kriterium mehr ist, führen jedoch mitunter dazu, dass sich der Zugang zu studienvorbereitenden Sprach- und Fachkursen, BAföG und studienbegleitenden Praktika verzögert oder bei einer Ablehnung durch die zuständige Ausländerbehörde gänzlich versperrt wird. Ob und inwiefern sich diese und weitere rechtliche Hürden im Einzelfall auswirken, kann der Regelübersicht in Tab. 3 entnommen werden.

Insgesamt zeigt die Analyse, dass Geflüchtete beim Übergang in den hiesigen Arbeitsmarkt mit sehr ähnlichen Herausforderungen konfrontiert sind wie ihre internationalen Mitstudierenden, die keine Fluchterfahrung aufweisen. Auch sie kämpfen mit sprachlichen Hürden, fehlender Praxiserfahrung und Schwierigkeiten beim Schreiben von Bewerbungen oder in Vorstellungsgesprächen (Abb. 1). Gruppenspezifische Unterschiede ergeben sich aus dem Bedarf nach zusätzlicher individueller Beratung und Betreuung, der bei den Geflüichteten in den Integra-Projekten vergleichsweise häufig entsteht. Denn anders als ihre internationalen Mitstudierenden haben Geflüchtete ihr Studium in Deutschland nicht jahrelang im Voraus geplant und nur die Allerwenigsten können auf finanzielle Hilfe durch ihre Familie hoffen. Auch die rechtlichen Unsicherheiten sind bei dieser Zielgruppe insgesamt höher. Zudem sollte erwähnt werden, dass geflüchtete Frauen, die insgesamt 40 Prozent des Teilnehmerkreises ausmachen (Tab. 8), besonders von Informationen und Beratung zu Themen wie strategischer Karriereplanung profitieren können, nicht zuletzt, weil viele von ihnen während oder im Anschluss des Studiums einen vergleichsweise höheren Aufwand für die Kinderbetreuung übernehmen (werden) als ihre männlichen Kommilitonen. ${ }^{18}$

\subsection{Schwerwiegendere Hürden für ältere und berufserfahrene Zielgruppen}

Ein knappes Drittel der Erwachsenen, die in den vergangenen Jahren hierzulande einen Antrag auf Asyl gestellt haben, hat bereits außerhalb Deutschlands studiert. 18 Prozent bringen einen Hochschulabschluss mit und haben i. d. R. als Fachkraft im Ausland gearbeitet (Brücker/Kosyakova/Schuß 2020: 4). Diese Hochqualifizierten können ein Gewinn für den hiesigen Arbeitsmarkt sein, in dem sich Fachkräfteengpässe zunehmend verschärfen. Dennoch schaffen viele es auch nach Jahren nicht, eine Arbeitsstelle

17 Neben den 4.233 Studierenden, die Anfang $2021 \mathrm{im}$ Studium eingeschrieben waren, haben 412 die Studienzusage nicht angenommen oder das Studium frühzeitig abgebrochen.

18 Dieser Aspekt wurde in den schriftlichen und mündlichen Antworten nur am Rande erwähnt, deckt sich aber mit der allgemeinen Befundlage (vgl. Middendorff et al. 2017: 81; Apolinarski/Brandt 2018: 48). 
zu finden, die ihrem Qualifikationsniveau entspricht und üben Tätigkeiten aus, für die sie überqualifiziert sind. Ein zunehmender Teil entschließt sich daher für eine Rückkehr ins Bildungssystem (Brücker et al. 2019: 11; Brücker/ Kosyakova/Schuß 2020: 9-10). So auch die 192 Teilnehmerinnen und Teilnehmer an den hochschulischen Weiterbildungsprogrammen, die an 17 Standorten im Rahmen von PROFI entwickelt wurden. 82 Prozent von ihnen sind nach Deutschland geflüchtet (Tab. 8 im Anhang).
Ihre Erfahrungen zeigen, dass die Herausforderungen für studierte und berufserfahrene Flüchtlinge zunächst sehr ähnlich gelagert sind wie bei den zuvor beschriebenen jüngeren internationalen Zielgruppen. Auch sie kämpfen oft mit

- einem fachsprachlichen Aufholbedarf,

- Wissenslücken über den deutschen Arbeitsmarkt,

- individuellen Diskriminierungserfahrungen,

- den vielerorts eher traditionell geprägten Einstellungspolitiken von Unternehmen sowie

- den (fluchtbedingten) Lücken in der eigenen Erwerbs- und Bildungsbiografie (s. Kap. 2.1).

ABB. 1 WEITVERBREITETE HÜRDEN BEIM BERUFSEINSTIEG FÜR AUSGEWÄHLTE INTERNATIONALE ZIELGRUPPEN AN DEUTSCHEN HOCHSCHULEN

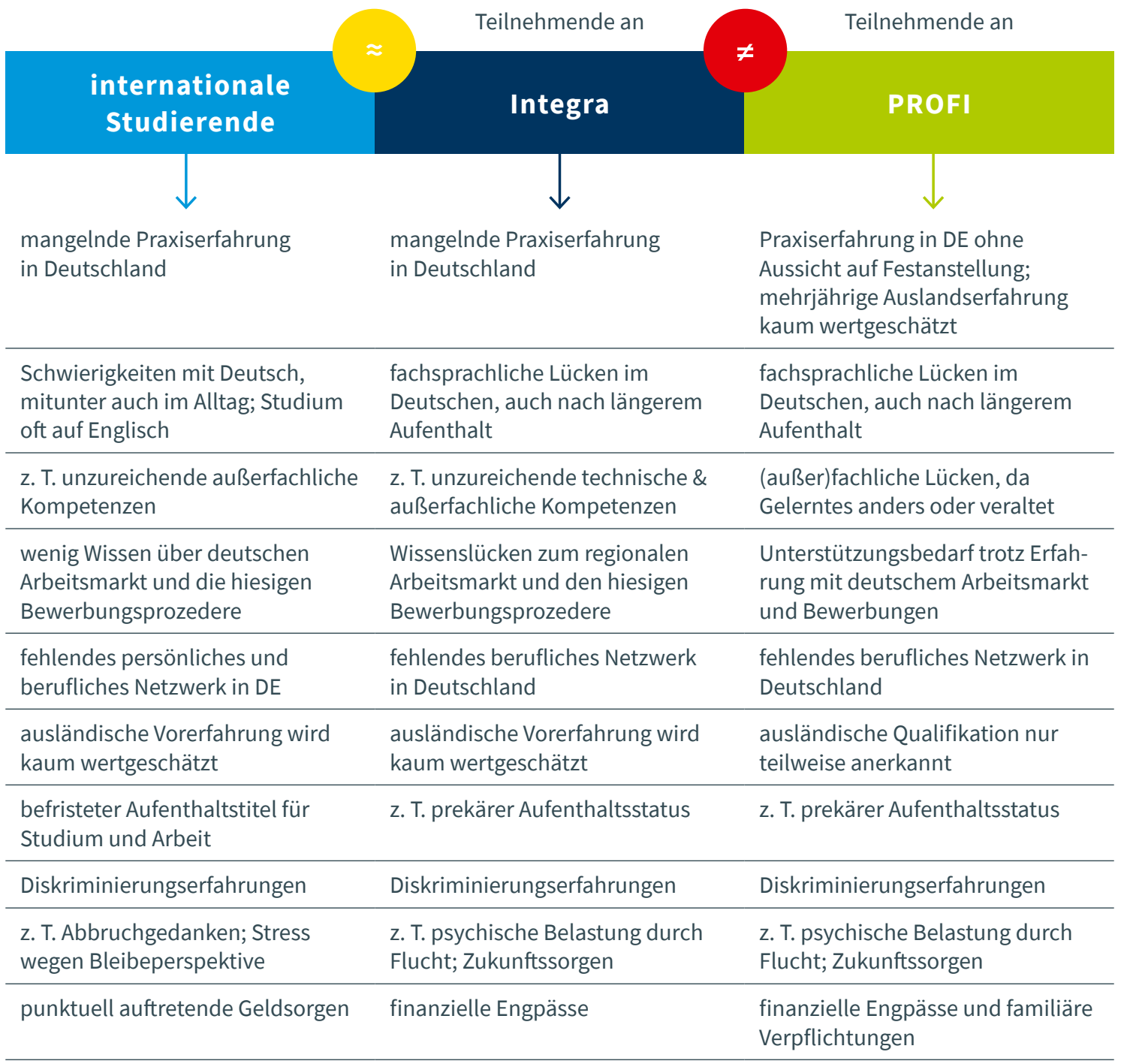

Zeitdruck und höhere Berufsansprüche aufgrund von Alter und Vorerfahrung 
Einem Großteil fällt die Bewältigung dieser Hürden - auf die im Folgenden nicht noch einmal gesondert eingegangen wird - allerdings deutlich schwerer als denjenigen, die gerade frisch im Studium ankommen. Dies ist trotz oder gerade wegen ihrer umfassenden Vorerfahrung der Fall und insbesondere auch aufgrund ihrer vergleichsweise größeren finanziellen und familiären Verpflichtungen (vgl. Engel/Wolter 2017: 71). Vor allem Frauen, die 39 Prozent des Teilnehmerfeldes ausmachen, müssen vergleichsweise mehr Zeit für die Kindererziehung aufwenden (vgl. Middendorff et al. 2017: 81; Apolinarski/ Brandt 2018: 48).

Unabhängig vom Geschlecht sehen viele in den PROFI-Projekten eine Chance, die sprachlichen, fachlichen und technischen Erwartungen in ihrem Wunschberuf möglichst schnell zu erfüllen. Da es sich dabei nicht nur um Einstiegsstellen handelt, sind ihre Ansprüche und ihr selbst Säule in Abb. 1). Folglich wiegen bürokratische Hürden sowie komplizierte und langwierige Anerkennungsverfahren für diese für viele Hochschulen neue Zielgruppe deutlich schwerer, wie die folgenden Ergebnisse zeigen.

\section{BÜROKRATISCHE HÜRDEN UND HOHE EIN- STIEGSVORAUSSETZUNGEN ERSCHWEREN ODER VERHINDERN EINE WEITERBILDUNG}

Über drei von vier Teilnehmenden in den PROFI-Projekten sind über 30 Jahre alt. Viele haben familiäre Verpflichtungen, bestreiten ihren Lebensunterhalt selbst und müssen somit abwägen zwischen Weiterbildung einerseits und einer möglichst schnellen Arbeitsaufnahme andererseits, wobei der sofortige Einstieg in eine niedriger qualifizierte Tätigkeit nicht selten zu eingeschränkten beruflichen Entwicklungsmöglichkeiten führt. Daher sehen die PROFI-Teilnehmenden in der Weiterbildung die Chance, mittelfristig eine abschlussadäquate Beschäftigung zu finden.

Obwohl die Weiterbildungsangebote an den projektteilnehmenden Hochschulen kürzer sind als ein Vollstudium, berichten viele der Geflüchteten, dass ihnen die Entscheidung für die Projektteilnahme schwergefallen sei. Viele sehen sich unter Zeitdruck und wünschen sich eine möglichst kurze Lerndauer, um den (Wieder-)Einstieg in den deutschen Arbeitsmarkt nicht noch weiter zu verzögern - nicht zuletzt, weil sie sich gegenüber jüngeren Berufsanwärterinnen und -anwärtern im Nachteil sehen:

\section{Wenn man den Bachelor-Abschluss macht in Deutschland, dann ist man vielleicht höchstens in Ausnahmefällen 25 oder so. Da bin ich dann 10 Jahre drüber, wenn es so weit wäre dann. Das ist vielleicht die größte Hürde. \\ (Syrischer Weiterbildungsteilnehmer an einer Fachhochschule, PROFI)}

\section{Diesen Oktober werde ich 50 Jahre alt und damit ich schneller in den Arbeitsmarkt kommen kann, muss ich schnell diesen Weg gehen. Und deswegen, je später ich auf diesen Weg komme, bekomme ich weniger Chancen auf dem Arbeitsmarkt. \\ (Iranischer Weiterbildungsteilnehmer an einer Fachhochschule, PROFI)}

Der empfundene Zeitdruck verstärkt die Hoffnung auf ein zeitlich begrenztes und möglichst unbürokratisch zugängliches Bildungsangebot. Die Hochqualifizierten haben nach eigener Ansicht bereits viel Zeit verloren und hadern mit dem bürokratischen ,Hürdenlauf', der seit ihrer Ankunft in Deutschland ihr Berufsleben und ihre bisherigen Bildungsanstrengungen maßgeblich mitbestimmt. Neben der Ausländerbehörde wird die Bundesagentur für Arbeit (BA) in diesem Zusammenhang häufig erwähnt. Ein Teil der PROFI-Teilnehmenden hat Vorerfahrung mit BA-Kursen oder hat im Vorfeld versucht, Zugang zu erhalten. Zwar waren Beratungsleistungen wie etwa zur Anerkennung der ausländischen Vorbildung uneingeschränkt zugänglich, jedoch war die Teilnahme an dem vielfältigen Qualifizierungsangebot der Bundesagentur und ihrer Trägerorganisationen nicht immer möglich:

Als ich hierhergekommen bin, war ich am Anfang beschäftigt mit meinem Asylverfahren und damals durfte ich nicht arbeiten eigentlich. Ich habe versucht, eine Arbeits- 
erlaubnis zu bekommen, aber das war ein bisschen schwer, man muss erst anerkannt sein, dann kann man arbeiten. Aber trotzdem habe ich ein Praktikum gemacht in einem Steinbruch für zwei Monate. Ich habe auch eine Stelle gefunden, ja, aber das Problem ist, dass ich einen ausländischen $\mathrm{Ba}$ chelor, einen ausländischen Abschluss habe, das ist ein Problem, das andere Problem war, dass ich keine Arbeitserlaubnis besitze. (Iranischer Weiterbildungsteilnehmer an einer Universität, PROFI)

Diese und andere Vorerfahrungen der Geflüchteten im PROFI-Programm verdeutlichen, dass das ordnungspolitische Ziel der Einwanderungssteuerung und -begrenzung, das sich in den differenzierten Zugangsregeln niederschlägt, teilweise in einem Spannungsverhältnis zu politischen und individuellen Integrationsbestrebungen steht. Zwar gibt es vielfältige Anerkennungs- und Qualifizierungsangebote für Neuzugewanderte. Die aus Sicht der Betroffenen undurchsichtigen Zugangsregeln können jedoch zu Verzögerungen auf dem Weg in den Arbeitsmarkt oder in eine staatliche Weiterbildung führen:

\footnotetext{
Ja, die Arbeitsagentur war nicht nur auf meiner Seite, manchmal ist es auch irgendwie vielleicht nur durch bürokratische Dinge negativ. [...] Zum Beispiel hatte ich schon zwei Jahre oder anderthalb Jahre nach meiner Ankunft in Deutschland eine Ausbildung bei einer Firma und ich habe auch schon ein Praktikum absolviert. [...] Und dann fange ich mit der Ausbildung an, was alles in Ordnung war. Und da wollte die [Firma] aber, dass die Agentur für Arbeit diese Einstiegsqualifikation teilweise zumindest mitfinanziert [...]. Da hat aber die Agentur für Arbeit gesagt, dass dadurch, dass ich einen Hochschulabschluss habe, sollte dieses Programm, diese Einstiegsqualifikation nicht für mich in Frage kommen und hat alles abgesagt. Damals war es eine riesige Chance, dass ich eine Fachkraft werde und eine deut sche Ausbildung habe. [...] Ich verstehe immer noch nicht, warum das damals so war. (Syrischer Weiterbildungsteilnehmer an einer Fachhochschule, PROFI)
}

Wie das Zitat verdeutlicht, müssen sich akademisch ausgebildete Geflüchtete nicht nur im behördlichen ,Dschungel' der Zuständigkeiten und Zugangsregeln orientieren, ihre Weiterbildungswünsche werden mitunter abgelehnt, weil sie als überqualifiziert gelten. Zudem bemängeln viele PROFI-Teilnehmerinnen und Teilnehmer rückblickend die Passung der außerhochschulischen Bildungsangebote. Statt einer allgemeinen Arbeitsmarktvorbereitung oder eines kompletten Ausbildungsgangs wünschen sie sich eine praxisorientierte, bedarfsgerechte Weiterbildung, die sie in möglichst kurzer Zeit für einen akademischen Beruf auf dem deutschen Arbeitsmarkt qualifiziert. Hinsichtlich ihrer zurückliegenden Weiterbildungsversuche waren sich die Befragten einig, dass ihnen die Weiterbildung über PROFI am ehesten eine erfolgreiche Berufsperspektive verspricht.

Doch auch diese Bildungschance ist nicht für alle Interessierten ein Einstiegsweg: Mehr als 60 Prozent der insgesamt 519 Bewerbungen im Wintersemester 2020/2021 wurden seitens der Hochschulen abgelehnt - die Ablehnungsquote ist damit knapp doppelt so hoch wie im Integra-Programm (Tab. 4). Die Gründe für die Ablehnungen lagen weniger in den verfügbaren Personal- und Sachmitteln vor Ort, sondern wie auch bei anderen Weiterbildungseinrichtungen vor allem an den Zugangsregeln bzw. der Tatsache, dass viele Interessierte die hohen Voraussetzungen nicht erfüllten: Oft hatten die studien- und berufserfahrenen Bewerberinnen und Bewerber ihr Studium im Ausland fluchtbedingt oder aus anderen Gründen unterbrochen und konnten somit nicht den für die Projektteilnahme erforderlichen Hochschulabschluss vorweisen. In begründeten Einzelfällen, in denen nur wenige Studienleistungen zu einem Abschluss fehlten, wurden mitunter Ausnahmeregelungen getroffen (Tab. 9 im Anhang). 


\section{AUSLÄNDISCHE VORERFAHRUNG OFT NUR SCHWER ÜBERTRAGBAR AUF DEN DEUTSCHEN ARBEITSMARKT}

Mehr als acht von zehn PROFI-Teilnehmenden verfügen über mehrjährige Arbeitserfahrung im Ausland, die auf ihrem dort absolvierten Erststudium aufbaut. Etwa die Hälfte hat bereits in Deutschland gearbeitet, jedoch meist nur in niedrigqualifizierten oder zeitlich befristeten Aushilfstätigkeiten oder Praktika. Eine adäquate Beschäftigung, die auf ihrem Fachwissen und ihren Fähigkeiten aufbaut, wurde bislang nur wenigen in Aussicht gestellt. Selbst eine formale Anerkennung der ausländischen Ausbildung führt oft nicht zum Wunschberuf. Ein wichtiger Grund dafür ist, dass sich die im Ausland erworbenen Fachkompetenzen in ihrem Umfang und ihrer Schwerpunktsetzung von den hiesigen Bildungsgängen unterscheiden. Die daraus entstandenen Lücken können oft nicht einfach durch formale Anerkennung wettgemacht werden und erfordern eine Rückkehr ins Bildungssystem:

\section{Was man in Syrien an Erfahrung hat im Be- reich Technik und Maschinenbau überhaupt ist im Vergleich zu Deutschland sehr gering. Deswegen habe ich viele Lücken in den In- halten gefunden. Die Lücke war nicht ganz einfach [...] zu schließen. Deswegen habe ich mich entschieden, es nochmal über das Studium zu versuchen. \\ (Syrischer Weiterbildungsteilnehmer an einer Fachhochschule, PROFI)}

In reglementierten Berufen treffen die Fachkräfte auf zusätzliche Eintrittsbarrieren. Zum Beispiel ist es für eine Tätigkeit im Bereich der Sozialen Arbeit i. d. R. erforderlich, dass die ausländische Ausbildung nicht nur formal anerkannt wird, sondern auch, dass die Gleichwertigkeit gegenüber ihrem deutschen Pendant vollumfänglich sichergestellt ist. Bundesweit wird bislang ein Großteil der Anerkennungsanträge im Bereich der Pflegeberufe eingereicht, für den hierzulande kein Studium vonnöten ist. Anträge im Bereich der Akademikerberufe sind vergleichsweise seltener und werden häufig mit Auflagen zur Nachqualifizierung belegt, weil das ausländische Studium nur einen Teil der hiesigen Ausbildung abdeckt, wie das obige
Zitat verdeutlicht (vgl. BIBB 2021: 15). Hierzu zählen ausländische Lehrkräfte, für die die Abschlussanerkennung durch die zuständigen Kultusbehörden eine hohe Hürde darstellt. Teilweise können lange Wartezeiten verstreichen, bis ein Anerkennungsverfahren abgeschlossen ist. Zudem müssen Lehrkräfte in den allermeisten Bundesländern zwei Fächer unterrichten können, was in vielen Fällen eine Weiterbildung erfordert. Die damit verbundene mangelnde unmittelbare Anerkennung der eigenen Studienund Berufserfahrung ist aus Sicht vieler Zugewanderter zwar verständlich, muss aber nicht nur auf fachlicher Ebene, sondern insbesondere auch emotional verarbeitet werden, wie das Personal in den PROFI-Projekten berichtet:

\section{Also es gibt diese formale Abwertung in Kombination damit, dass sich ein solches [abwertendes] Gefühl einstellt. Also unse- re Leute, mit denen wir arbeiten, eine der Hauptherausforderungen ist, sie wieder aufzurichten und ihnen Mut zu machen für einen schweren Weg. (Mitarbeiterin an einer Fachhochschule, PROFI)}

Sowohl das Personal als auch die PROFI-Teilnehmenden betonen, dass letztlich die Einstellungspraxis auf dem Arbeitsmarkt darüber entscheidet, welchen Wert die ausländische Vorerfahrung hat. Zwar kann eine formale staatliche Abschlussanerkennung die Transparenz erhöhen, doch auch wenn diese vorliegt, kann es für Arbeitgeberinnen und Arbeitgeber schwierig sein, die fachliche Passung einer ausländischen Ausbildung einzuschätzen (vgl. Jacob/Hermann/ Schmidt 2014). Folglich dürften internationale Fachkräfte auch weiterhin ein reges Interesse an hochschulischer Weiterbildung haben:

Ich denke, für jemand mit einem ausländischen Abschluss ist es schwer, hier einen Job zu finden. [...] Aber ein deutscher $A b$ schluss oder europäischer Abschluss, denke ich, die haben Priorität, dann kommen andere Länder wie Orient oder die anderen Länder. Ich denke, das ist das Problem, das viele Flüchtlinge hier haben. [...] Mein Abschluss zum Beispiel wurde anerkannt offiziell bei einer Behörde. Ich denke, ja, 
es hängt vom Arbeitgeber oder den Chefs einer Firma ab, ja, sie wissen nicht genau, was wir in unseren Ländern machen. Vielleicht ist es unbekannt für sie, vielleicht sie können nicht einfach vertrauen.

(Iranischer Weiterbildungsteilnehmer an einer Universität, PROFI)

Insgesamt zeigt der Vergleich der Erfahrungen der verschiedenen internationalen Zielgruppen in den Programmen Integra und PROFI, dass individuelle Fluchtbiografien nicht automatisch zu neuen Herausforderungen beim Übergang vom Hörsaal in den Betrieb führen - sieht man von spezifischen Bedarfen im Bereich der Beratung zum Umgang beispielsweise mit asylrechtlichen Rahmenbedingungen einmal ab. Gleichwohl ergeben sich andersartige Herausforderungen für diejenigen, die im höheren Alter und mit mehrjähriger qualifizierter Berufserfahrung nach Deutschland zuwandern. Sie benötigen weniger eine allgemeine Arbeitsmarktvorbereitung, sondern vielmehr eine spezialisierte Weiterbildung oder Anpassungsqualifizierung: Sie brauchen ein akademisches Lernangebot, das zeitlich komprimiert und präziser abgestimmt ist auf die konkreten Anforderungen in einzelnen Zielberufen und -branchen (vgl. Damelang/Kosyakova 2021). Dies verdeutlichen die Erfahrungen aus dem PROFIProgramm, dessen Pilotierung verschiedener Weiterbildungskurse für auslandserfahrene Lehrkräfte, IT-Spezialisten und andere internationale Fachkräfte einen Vorbildcharakter haben könnte. Für diese dürfte zukünftig ein wachsender Bedarf bestehen, der sich nicht nur aus dem Zuzug von Flüchtlingen ergibt, sondern beispielsweise auch aus dem Bedarf von Menschen, die gezielt zum Zwecke der Weiterbildung oder im Rahmen des Familienmit- und -nachzugs mit ihren erwerbstätigen Partnern oder Partnerinnen nach Deutschland kommen (vgl. Borowsky et al. 2020: 25-32). 


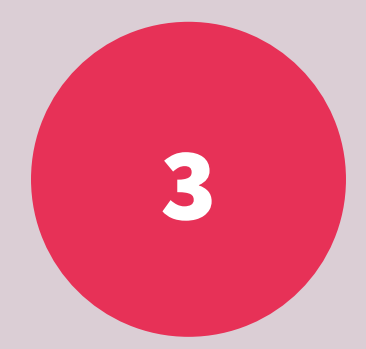

\section{Wegbereitung in den Arbeitsmarkt zumeist digital: erste Erfolge und zukünftiger Entwicklungsbedarf}

3.1 Pragmatische Umstellung auf digitale Formate.

3.2 Digitale Formate: Herausforderung und Mehrwert zugleich 
Seit Anfang 2020 wird der Hochschulalltag stark von der Corona-Pandemie bestimmt. Auf die Frage, wie diese das Kurs- und Beratungsgeschehen an den Hochschulen beeinflusst, gibt es kaum eine Antwort, in der das Wort „Digitalisierung" nicht als erstes fällt. Trotz der gravierenden allgemeinen Einschränkungen ist der Studienbetrieb kaum ins Stocken geraten. Seit Pandemiebeginn finden rund 90 Prozent des Lehrbetriebs über digitale oder hybride Formate statt. Die große Mehrheit der Studierenden kann somit auch weiterhin die geplanten Vorlesungen, Seminare und Tutorien besuchen, i. d. R. von daheim über Videokonferenzen und Vorlesungsaufzeichnungen (DZHW 2020: 2; Stifterverband 2020: 3). Die vielerorts geplanten Praxiserfahrungen im Rahmen sog. ServiceLearning-Angebote ${ }^{19}$ konnten aufgrund der strengen Hygiene- und Kontaktregeln nur sehr eingeschränkt stattfinden und werden im Folgenden nicht gesondert betrachtet.

An den meisten Hochschulen erfolgte die ,Express-Digitalisierung' in nur einem Monat dank tatkräftiger Unterstützung der Rechenzentren, E-Learning-Büros, Bibliotheken und weiterer Einrichtungen innerhalb der Hochschulen eine Leistung, die von den Studierenden und dem Personal zunächst sehr begrüßt wurde (Stifterverband 2020: 2-3; Goertz/Hense 2021: 18-19). Gleichzeitig ist aber bislang nicht hinreichend geklärt, wie geeignet die derzeit genutzten digitalen Formate für benachteiligte Zielgruppen sind, zu denen auch Geflüchtete und andere internationale Studierende zählen (DZHW 2021a).

Die Erfahrungen aus Integra und PROFI liefern dahingehend einen ersten Einblick und zeigen, wie digitale und hybride Unterstützungsangebote bislang eingesetzt werden, um Geflüchteten den Weg in den deutschen Arbeitsmarkt zu ebnen (s. Kap. 3.1) ${ }^{20}$ und welche Herausforderungen und Chancen sich daraus für die Teilnehmenden und das Hochschulpersonal ergeben (s. Kap. 3.2). Da im Rahmen der vorliegenden Begleitstudie keine groß angelegte Studierendenbefragung durchgeführt wurde, stützen sich die folgenden Befunde vor allem auf die Eindrücke der befragten Mitarbeiterinnen und Mitarbeiter in den 88 untersuchten Hochschulprojekten.

\subsection{Pragmatische Umstellung auf digitale Formate}

Der Erfolg im Studium und beim Berufseinstieg hängt in erster Linie vom individuellen Engagement der Lernenden ab. Doch es gibt zahlreiche Unterstützungsangebote: Insgesamt unterbreiten die Integra- und PROFI-geförderten Projekte mehr als 500 Angebote zum Arbeitsmarkteinstieg. Die Mehrheit dieser Angebote wurde in der laufenden Förderphase neu entwickelt (Tab. 10 im Anhang). Neben Bewerbungsmappen-Checks und Bewerbungsworkshops setzen die Hochschulen vor allem auf Seminare, etwa zur Berufspraxis in ausgewählten Branchen, oder auf Gastvorträge durch Unternehmen, freiberufliche Karrierecoaches und andere Partnerinnen und Partner (Tab. 10 im Anhang). Zudem bieten die Hochschulen eine oft enge individuelle Betreuung und Beratung, z. B. wenn es um Berufsorientierung, Finanzierungsmöglichkeiten oder die Anerkennung ausländischer Abschlüsse geht.

Diese Veranstaltungen und die flankierenden Beratungs- und Betreuungsleistungen wurden seit Pandemiebeginn größtenteils in den digitalen Raum verlagert. Über 90 Prozent der Arbeitsmarktvorbereitung erfolgt seither über digitale oder hybride Formate (Abb. 2). Präsenzangebote konnten bislang nur in Einzelfällen und unter strengen Hygiene- und Verhaltensregeln zwischen den Lockdown-Phasen stattfinden. Das Gros der Wegbereitung in den deutschen Arbeitsmarkt erfolgt somit auch weiterhin primär am Bildschirm. Punktuelle Ausnahmen wurden bis dato am ehesten für individuelle

19 Beim Service Learning engagieren sich Studierende für das Gemeinwohl und erhalten somit wertvolle praktische und fachliche Einblicke. Die Aktivitäten bauen auf den theoretischen Lerninhalten des Studiums auf und werden idealerweise als Studienleistung anerkannt (SVRForschungsbereich 2016b).

20 Die Mehrheit der Teilnehmerinnen und Teilnehmer in den untersuchten Integra- und PROFI-Projekten ist vor Jahren nach Deutschland geflüchtet. Ihre Erfahrungen mit digitalen Unterstützungsangeboten dürften wichtige Hinweise auf die Situation anderer internationaler Studierender geben, die oft vor ähnlichen Hürden stehen (s. Kap. 2.1) 
Beratungsgespräche gemacht, z. B. in persönlichen Krisensituationen:

\section{Unsere Beratung findet online statt. Wir haben einzelne sensiblere Beratungssitua- tionen gehabt, für die wir ein Walk-and-Talk- Format angeboten haben. Also, Treffen vor der Uni, Spaziergang, einmal rund um die In- nenstadt, um etwas zu besprechen. Aber das ist eher die Ausnahme. Es wird auch nicht sehr stark nachgefragt. Das ist für sensible Themen. In der Regel [beraten wir] virtuell. (Mitarbeiter an einer Universität, Integra-Programm)}

\section{Und das war auf jeden Fall viel schwieriger} für die Leute damit umzugehen mit dieser Online-Lehre und null Kontakt mit Kommilitoninnen und null Kontakt mit Professorinnen und wissenschaftlichen Mitarbeitern, alles online. Also man fühlt die Leute nicht und man sieht die Leute nicht. Und wir haben versucht, uns daran anzupassen. [...] Tatsächlich habe ich mich immer mal wieder auch mit ihnen individuell getroffen zum Spazierengehen, aber nicht in einem Raum, das heißt, wir haben auch viel online gearbeitet. (Mitarbeiterin an einer Fachhochschule, PROFI)

Wenngleich einige wenige Angebote von vornherein im digitalen oder Blended-Learning-Format geplant waren, mussten die allermeisten Unterstützungsleistungen kurzfristig umstrukturiert werden. Die konkrete Umsetzung war und ist dabei von Hochschule zu Hochschule unterschiedlich. An den meisten werden Bewerbungsworkshops und andere Angebote über Videokonferenzen durchgeführt (91\%) (Tab. 5). Diese sind nicht nur relativ schnell umsetzbar, sondern können das individuelle Lernen positiv beeinflussen, wie eine Befragung von rund 25.000 (größtenteils deutschen) Studierenden zeigt, die das Deutsche Zentrum für Hochschul- und Wissenschaftsforschung (DZHW) im Sommersemester 2020 durchgeführt hat: Je häufiger Videokonferenzen im besagten Semester zum Einsatz kamen, desto besser konnten die Studierenden dem Lernstoff folgen und ihr eigenes Lernen selbst organisieren (DZHW 2021b: 5). Gleichwohl kritisierte ein Teil des ansonsten überwiegend experimentierfreudigen Lehrpersonals die mangelnde Bereitschaft vieler Studierender, sich aktiv an den Diskussionen innerhalb der Videokonferenzen zu beteiligen (Seyfeli/Elsner/Wannemacher 2020: 5). Dies überrascht insofern kaum, als didaktische Konzepte aufgrund der kurzfristigen Einführung von videogestützter Lehre bislang nur selten auf das mittlerweile sehr gängige Videokonferenzformat abgestimmt werden konnten (Stifterverband 2020: 8). Auch ist ungeklärt, welcher Teil der Studierenden die besagten positiven Lerneffekte tatsächlich erzielt. Insgesamt gibt etwa ein Drittel aller Studierenden an, dass sich ihre Lernsituation seit Beginn der Pandemie deutlich verschlechtert hat (DZHW 2021b: 4). Geflüchtete Studierende und andere internationale Zielgruppen, die eigens

\section{ABB. 2 ARBEITSMARKTVORBEREITUNG IN ZEITEN DER CORONA-PANDEMIE}

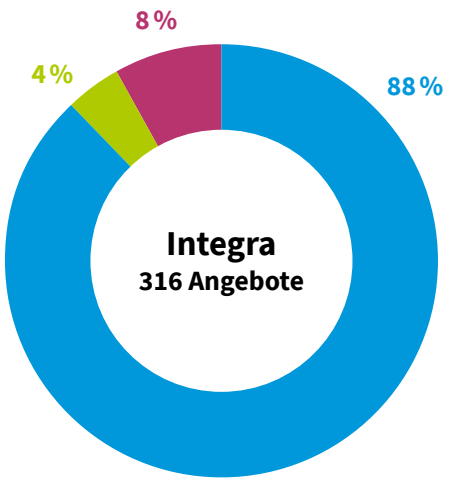

digitales Format
Blended-Learning-Format

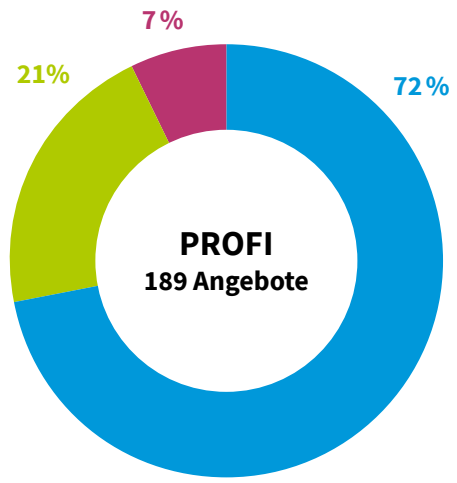

Präsenzformat 
TABELLE 5: EINSATZ DIGITALER LEHR- UND LERNTECHNOLOGIEN IM INTEGRA-PROGRAMM

\begin{tabular}{|c|c|}
\hline Anteil der Hochschulen & eingesetzte Technologien \\
\hline mehr als $50 \%$ & $\begin{array}{l}\text { Videokonferenzen (91 \%) } \\
\text { Lehrvideos (70 \%) } \\
\text { Lernplattformen (61 \%) } \\
\text { Online-Evaluationen }(59 \%) \\
\text { digitale Tafeln }(54 \%) \\
\text { Webinare }(51 \%)\end{array}$ \\
\hline 25 bis $50 \%$ & $\begin{array}{l}\text { soziale Medien (43\%) } \\
\text { offen zugängliche Bildungsmaterialien („OER“) (41 \%) } \\
\text { Webanwendungen („Apps“) (39\%) } \\
\text { Online-Foren (38\%) } \\
\text { virtuelle Klassenzimmer (36 \%) } \\
\text { Podcasts (36 \%) } \\
\text { Vorlesungsaufzeichnungen ( } 29 \%) \\
\text { elektronische Klausuren (26\%) }\end{array}$ \\
\hline weniger als $25 \%$ & $\begin{array}{l}\text { elektronische Lernportfolios (14 \%) } \\
\text { web-basierte Trainingseinheiten (13\%) } \\
\text { Online-Wissenssammlungen („Wikis“) (10\%) } \\
\text { studioproduzierte Vorlesungen (7 \%) } \\
\text { offen zugängliche Online-Kurse („MOOCs“) (3\%) } \\
\text { computersimulierte Lernumgebungen („,VR/AR“) (0 \%) }\end{array}$ \\
\hline
\end{tabular}

Quelle: Eigene Auswertung der Daten aus Integra

zum Studium zugewandert sind, werden in diesen Analysen bislang nicht gesondert ausgewiesen,21 dürften aber besonders betroffen sein (s. Kap. 3.2).

Neben Videokonferenzen nutzt das Personal in den Integra-Projekten ${ }^{22}$ vor allem Lehrvideos (70 \%) und digitale Lernplattformen (61\%), um die Studierenden auf ihren späteren Berufseinstieg vorzubereiten. Beide Technologien waren bereits vor der Pandemie an nahezu allen Hochschulen vorhanden und somit schnell einsetzbar (vgl. Ladwig 2019). Für Studierende ohne muttersprachliche Deutsch- oder Englischkenntnisse bieten sie den großen Vorteil, dass Lerninhalte beliebig häufig und in eigenem Tempo wiederholt werden können (DZHW 2021b: 5). Ein kleinerer Teil der projektteilnehmenden Hochschulen setzt zudem auf soziale Medien (43\%), Lern-Apps (39\%) oder Podcasts (36\%). Aufwendigere Technologien wie studioproduzierte Vorlesungen (7\%) oder computersimulierte Lernumgebungen (0 \%) kommen bislang so gut wie gar nicht zum Einsatz (Tab. 5).
Die Erfahrungsberichte des projektverantwortlichen Personals zeugen von demselben Pragmatismus, der handlungsleitend für die pandemiebeschleunigte Digitalisierung der Hochschullehre war (vgl. Hochschulforum Digitalisierung 2020; Stifterverband 2020; Goertz/Hense 2021; DZHW 2020). Insgesamt zeigen sich die Projektverantwortlichen relativ zufrieden mit der spontanen Umstrukturierung ihrer Vorhaben, nicht zuletzt weil einige bereits von vornherein digitale und hybride Lernelemente enthielten.

Also, wir mussten natürlich ein bisschen justieren. Die Kernelemente konnten wir alle erfolgreich digitalisieren. Das heißt Sprachkurse, den Lehrgang, unser Job-AlumniMentoring-Programm haben wir über eine [digitale] Plattform realisieren können. (Mitarbeiter an einer Universität, Integra-Programm)

21 In seiner Sonderauswertung zu besonders gefährdeten Studierendengruppen erwähnt das DZHW u. a. auch internationale Studierende, geht darin aber lediglich auf das Stressempfinden der Lernenden ein, welches genau wie bei deutschen Studierenden im Sommersemester 2020 sehr hoch war (DZHW 2021a: 7).

22 Der Einsatz verschiedener Lerntechnologien wurde in den PROFI-Projekten nicht im Detail erfragt. 
Nein, das ist sehr seltsam, dass ich irgendwie im Januar 2020 einen Antrag geschrieben habe, [...] der einen sehr schweren Digitalisierungsfokus hat. Weiß ich auch nicht, wie das entstanden ist. Wir wollten eben Online-Lehre produzieren, weil wir seit vier Jahren feststellen, ,Punktuell wiederhole ich das Gleiche: Und wir wollten das jetzt endlich in Videoformate packen und anfangen, so nach Blended-Learning-Prinzip, den Unterricht dann an solche eigenständigen Lerneinheiten digital anzukoppeln.

(Mitarbeiterin an einer Universität, PROFI)

Trotz oder gerade wegen des beachtlichen Tempos bei der Umstellung führt der Online-Betrieb bisweilen zu neuen Herausforderungen für die Lernenden und das Hochschulpersonal, wie das folgende Kap. 3.2 zusammenfasst.

\subsection{Digitale Formate: Herausforde- rung und Mehrwert zugleich}

Nicht nur das Hochschulpersonal in den Integraund PROFI-Projekten ist im Zuge der digitalen Umstellung stark gefordert. Insbesondere die Geflüchteten stellt der ,Digitalisierungsturbo“ vor neue Herausforderungen: Nicht alle verfügen über die notwendige technische Ausstattung, einen ruhigen Lernort oder eine schnelle Internetverbindung. ${ }^{23}$ Diese Rahmenbedingungen entscheiden jedoch maßgeblich darüber, wie regelmäßig Studierende an Online-Angeboten teilnehmen (vgl. DZHW 2021b: 7). Zwar berichten weder die Geflüchteten noch das Personal von einem breiten Rückgang der Teilnahmebereitschaft, doch der Mangel an leistungsfähigen Endgeräten und stabilen Netzzugängen erschwert bisweilen den Lernbetrieb:

\footnotetext{
Allerdings merkt man doch häufiger in den Seminaren, dass die Internetverbindungen unzureichend sind, keine Kameras vorhanden sind oder eben auch Störfaktoren durch Mitbewohner vorhanden sind.

(Mitarbeiterin an einer Fachhochschule, Integra-Programm)
}

Manche Teilnehmerinnen und Teilnehmer wohnen auch nach wie vor in Gemeinschaftsunterkünften, in denen es kein WLAN gibt. Andere wohnen auf engem Raum mit ihrer Familie zusammen, sodass ihnen die Nutzung der Videofunktion teilweise unangenehm war, da Familienmitglieder im gleichen Raum und anderweitig beschäftigt waren. (Mitarbeiter an einer Universität, Integra-Programm)

Das Studium im digitalen und Blended-Learning-Format ist sowohl für geflüchtete als auch für einheimische Studierende nach wie vor ungewohnt und sehr zeitaufwendig. Nur wenige kommen mit den für das digitale Lernen notwendigen technischen, kommunikativen und gestalterischen Fähigkeiten an die Hochschule und müssen sich somit das Lernen an sich stückweise neu aneignen, z. B. wie online verfügbare Informationen kritisch hinterfragt und kreativ genutzt oder wie sensible Lerndaten geschützt werden können (vgl. Hochschulforum Digitalisierung 2020: 24-28).

Die Geflüchteten in den Integra- und PROFI-Projekten stehen hier vor besonders großen Herausforderungen. Einerseits haben sie oft weniger Vorerfahrung mit digitalen Lernplattformen und Lerntechnologien als diejenigen, deren Studium in Deutschland länger im Voraus geplant war. Somit ist die technische Einarbeitung oft sehr zeitaufwendig für sie. Andererseits erfordern digitale Lernformate zum Teil eine hohe Lese- und Schreibkompetenz sowie ein gutes sprachliches Hörverständnis, was die ohnehin schon bestehenden sprachlichen Hürden weiter erhöht. Ohne sprachliche und didaktische Anpassung kann die mündliche Beteiligung der Geflüchteten in digitalen Seminaren und Workshops daher noch stärker gehemmt werden als im Präsenzbetrieb (vgl. SVR-Forschungsbereich 2017b: 21-22; 2019: 32-36).

23 Zum Vergleich: Deutschlandweit berichten 86 Prozent der Studierenden, dass sie keine technischen Probleme bei der Nutzung der digitalen Lehrangebote haben (DZHW 2020: 4). 
Da die zügige Digitalisierung inner- und außerhalb der Projekte bislang eher auf das kurzfristig Machbare abzielte und weniger auf den tatsächlichen didaktischen Bedarf einzelner Studierendengruppen, erscheint eine dahingehende Weiterentwicklung notwendig, damit auch Geflüchtete und andere Studierende ohne muttersprachliche Deutsch- und Englischkenntnisse voll von der digitalen Lehre und Arbeitsmarktvorbereitung profitieren können (vgl. Stifterverband 2020: 15-17). Hierzu müssen auch die digitalen Lehrkompetenzen des Hochschulpersonals geschärft werden. Knapp die Hälfte unterrichtet zum ersten Mal online und ist oft noch nicht hinreichend für die zusätzlichen Hürden sensibilisiert, vor denen internationale Kursteilnehmerinnen und -teilnehmer stehen (vgl. Hochschulforum Digitalisierung 2020: 25). Insgesamt empfindet das Lehrpersonal in den berufsvorbereitenden Seminaren, dass es ohne direkten Präsenzkontakt zu den Lernenden deutlich schwieriger ist, Unklarheiten zu identifizieren, wirksam Rückmeldung zu geben und individuell zu begleiten. Zwar wird in mehr als der Hälfte der Projekte mit Online-Tafeln, virtueller Kleingruppenarbeit oder Online-Selbsttests experimentiert (Tab. 5), der souveräne Umgang mit diesen und anderen digitalen Werkzeugen erfordert jedoch eine systematische Weiterqualifizierung des Personals und eine stärkere Zusammenarbeit innerhalb der Hochschulen, nicht zuletzt, um die verwirrende Vielzahl an parallel genutzten Technologien zu begrenzen (vgl. Seyfeli/Elsner/Wannemacher 2020: 86-87).

Das Hochschulpersonal in den Integra- und PROFI-Projekten verweist zudem auf die große Bedeutung von sozialen Kontakten, deren Aufbau in Online-Seminaren deutlich schwerer fällt als in Präsenzveranstaltungen (vgl. DZHW 2020: 4). Diese Kontakte sind nicht nur wichtig für das Ankommen im Studium; sie können außerdem mittelfristig beim Deutschlernen, bei der Jobsuche oder beim Schreiben von Bewerbungen helfen und sind somit ein entscheidender Faktor beim Einstieg in den Arbeitsmarkt, wie der SVR-Forschungsbereich (2017a) bereits vor einigen Jahren herausgearbeitet hat. Auch der persönliche Kontakt zwischen den Lernenden und dem Hochschulpersonal ist online schwerer zu realisieren. Die Geflüchteten sind somit noch stärker auf sich allein gestellt als im Präsenzstudium, welches hierzulande bereits für viele ein ungewohnt hohes Maß an Selbstorganisation erfordert (vgl. Seyfeli/Elsner/Wannemacher 2020: 3$):^{24}$

\section{Digitale Elemente ermöglichen freiere \\ Zeiteinteilung, aber verlangen auch mehr Selbstdisziplin und Selbstorganisation. [Das] war für einige Studierende schwer umzusetzen. \\ (Mitarbeiterin an einer Universität, Integra-Programm)}

\section{Für die Zielgruppe der Geflüchteten und} auch die der Internationalen ist die Gefahr, in die soziale Isolation zu rutschen, höher als bei deutschen Studierenden, da ihnen oft ein stabiles soziales Umfeld noch fehlt. Präsenzlehre ist daher auch für das Ankommen, Freunde kennenlernen, sich ein soziales Netz schaffen von hoher Wichtigkeit und kann von digitalen Formaten nicht annähernd ersetzt werden. Gerade die Zielgruppe der Geflüchteten ist durch traumatische Erlebnisse zuweilen psychisch vorbelastet, was durch soziale Isolation oft verschlimmert wird. (Mitarbeiterin an einer Fachhochschule, Integra-Programm)

Das zweite Zitat unterstreicht die Mehrfachbelastung von Geflüchteten. Ohne eine persönliche Unterstützung laufen viele Gefahr, ihre Aus- und Weiterbildung frühzeitig abzubrechen, was ihre Chancen auf einen erfolgreichen Berufseinstieg deutlich verschlechtert. Derartige Abbruchgedanken konnten in den untersuchten Projekten bislang zwar nur punktuell festgestellt werden, in vielen Fällen wird aber davon ausgegangen, dass die Geflüchteten länger studieren werden als ursprünglich geplant (vgl. Lörz 2020;

24 Auch die Lernkultur ist in Deutschland zum Teil eine andere als in den Herkunftsländern. Viele der Neuzugewanderten müssen daher eine ganz neue und in vielen Fällen deutlich selbständigere Art des Studierens erlernen. Beispielsweise ist es hierzulande üblich, in Seminaren und Workshops mit dem Lehrpersonal und den Studierenden zu diskutieren. Vielen ist das unbekannt und zum Teil auch unangenehm, insbesondere, wenn sie allein vor dem Bildschirm lernen (vgl. SVR-Forschungsbereich 2017b: 21-25; Seyfeli/Elsner/Wannemacher 2020: 5). 
Grüttner/Schröder/Berg 2020). Mehr als zwei Drittel fühlen sich von ihrer derzeitigen Studienund Lebenssituation gestresst und leiden unter den nach wie vor unsicheren Aussichten auf ein Praktikum, eine Arbeitsstelle oder die Rückkehr zur Präsenzlehre (vgl. DZHW 2021: 6-7):

Besonders der nun bereits sehr lang andauernde Ausfall des Präsenzunterrichts hat bei vielen Teilnehmerinnen und Teilnehmern zu einem Einbruch der Motivation geführt. Hinzu kommt hier außerdem, dass pandemiebedingt auch einige bewerbungs- und studiengangsbezogene Unsicherheiten bestehen. (Mitarbeiter an einer Fachhochschule, Integra-Programm)

Ich weiß, dass Leute Schwierigkeiten hatten, Praktikumsstellen zu finden, natürlich, weil die Firmen zumindest am Anfang der ganzen Pandemie erstmal aus Sicherheitsgründen gesagt haben, ,Wir nehmen erstmal keinen.'

(Mitarbeiterin an einer Fachhochschule, Integra-Programm)

Das Interesse von Unternehmen an Kooperationen ist aufgrund der Corona-Pandemie drastisch eingebrochen. Sie bieten bspw. fast keine Praktika mehr an und haben kein Interesse an Veranstaltungen, da weniger Bedarf an Nachwuchs besteht und Konzepte für digitale Formate fehlen.

(Mitarbeiterin an einer Fachhochschule, Integra-Programm)

Viele der Herausforderungen, die sich aus der Umstellung auf digitale und hybride Formate ergeben, werden vom Personal allerdings nicht automatisch negativ bewertet, sondern zugleich als Chance und Mehrwert verstanden. So erlaubt die Abkehr vom Präsenzunterricht, dass Kursinhalte einfacher aufgezeichnet und somit zeit- und ortsunabhängig und deutlich früher im Studium einem breiten Teilnehmerkreis zur Verfügung gestellt werden können (vgl. DZHW 2021: 4-5). Geflüchtete, die ihr Praktikum an einem anderen Ort absolvieren, können somit beispielsweise auch weiterhin an virtuellen Sprachkursen und Bewerbungstrainings teilnehmen. Das gilt auch für diejenigen, die (noch) nicht direkt am Hochschulstandort leben und deren Mobilität aufgrund ihrer familiären und finanziellen Situation eingeschränkt ist:

Studierende mit Fluchthintergrund sind oft durch den erhöhten Aufwand im Studium und diverser Nebenjobs zeitlich sehr belastet. Bei digitalen Formaten sind sie eher bereit, abends noch an Workshops teilzunehmen, wenn der Weg zur Hochschule entfällt. Bei bestimmten digitalen Formaten können die Studierenden sich ihre Zeit frei einteilen und sich dann mit dem Thema beschäftigen, wenn es in ihren Tagesablauf passt, was dieser Zielgruppe auch zugutekommt. (Mitarbeiterin an einer Fachhochschule, Integra-Programm)

Viele Frauen mit Kindern haben sich positiv zum Online-Format geäußert. Viele hätten einen Präsenzkurs nicht machen können. (Mitarbeiter an einer Fachhochschule, Integra-Programm)

Auch was den sprachlichen und fachlichen Aufholbedarf vieler Geflüchteter angeht, bieten digitale und hybride Formate zusätzliche Lernmöglichkeiten. Durch die vielerorts genutzten Seminaraufzeichnungen und Lehrvideos können Lerninhalte beliebig oft abgespielt und wiederholt werden - ein Angebot, das von vielen begrüßt und mit Erfolg genutzt wird:

Der Mehrwert der digitalen Elemente ergibt sich aus der mehrfachen Nutzung der Materialien, die den Lernenden sowohl zeit-als auch ortsunabhängig zur Verfügung stehen und damit eine Wiederholung nach eigenem Lerntempo und Zeitbudget erlauben. Diese Flexibilisierung haben insbesondere Geflüchtete mit Familien genutzt und geschätzt. (Mitarbeiter an einer Fachhochschule, Integra-Programm) 
Durch die Online-Kommunikation haben die Teilnehmerinnen und Teilnehmer gelernt, sich noch präziser auszudrücken, digitale Hilfsmittel zu nutzen und digitale Programme und Geräte optimal einzusetzen. Ihnen gelingt es inzwischen sehr gut, mögliche Missverständnisse - einschließlich kultureller-, Kommunikationsprobleme und emotionale Reaktionen in Online-Diskussionen vorherzusehen und erfolgreich damit umzugehen.

(Mitarbeiterin an einer Universität, PROFI)

Neben der Erprobung neuer Formate für das Sprachenlernen kann die regelmäßige Nutzung verschiedener digitaler Lerninstrumente entscheidend dazu beitragen, dass Geflüchtete deutlich kompetenter im Umgang mit digitaler Technik sowie in virtuell arbeitenden Teams agieren - zwei Grundvoraussetzungen für eine Arbeitswelt, in der der tägliche Gang ins Büro in vielen Branchen unter Umständen eher die Ausnahme als die Regel bleiben wird (Taapken/ Heinen 2021; Erdsiek/Elbert 2021; Bitkom 2020). Der individuelle Aufbau von Computerkenntnissen, unterschiedlichen digitalen Kommunikationstechniken und weiteren berufsrelevanten Medienkompetenzen erfordert daher zusätzliche Unterstützung, doch auch hier verweisen mehrere Hochschulmitarbeiterinnen und -mitarbeiter auf das große Potenzial digitaler und hybrider Lernszenarien:

\section{Die Verlagerung der Lehrsequenzen in die} Eigenverantwortung von Lerngruppen führt zu mehr Unterrichtszeit, in der die internationalen Studierenden schwierige Inhalte und Themen ansprechen und Fragen direkt klären können. Auf diese Weise findet eine individuellere Betreuung durch die Dozentinnen und Dozenten statt.

(Mitarbeiter an einer Universität, Integra-Programm)
Was jedoch als Mehrwert bleibt, ist die Erfahrung, dass vor allem hybride Formate besonders effektiv sind und sowohl ein Gruppengefühl und Zusammenhalt sowie Vertrauen entstehen lassen, als auch individuelle Bedürfnisse und das Eingehen auf einzelne Lernfortschritte ermöglichen. Vor allem wenn nur in großen Lerngruppen im Präsenzformat gelehrt und gelernt wird, treten oft die individuellen Bedürfnisse hinter die der Gruppe zurück.

(Mitarbeiterin an einer Fachhochschule, Integra-Programm)

Insgesamt verdeutlichen die Erfahrungen in den Integra- und PROFI-Projekten, dass es bei der heiß geführten Diskussion um Digitalisierung in Zukunft weniger um das $\mathrm{Ob}$, sondern vielmehr um das Wie gehen sollte. Neben einer didaktischen Weiterentwicklung der zahlreichen neu entstandenen Online-Angebote versprechen insbesondere die hybriden Lernformate einen zusätzlichen Gewinn für internationale Zielgruppen. Durch eine gezielte Kombination aus flexibel zugänglichen OnlineKursen und persönlichen Beratungsgesprächen, Präsenzworkshops und Begegnungen mit Unternehmen (z. B. über Messen oder Exkursionen) können Zugewanderte aus Sicht des projektverantwortlichen Personals noch stärker entlang ihrer persönlichen Ausgangslagen für eine ebenfalls zunehmend flexible Arbeitswelt vorbereitet werden.

Auch für die Kooperation mit Unternehmen und anderen Partnerorganisationen am Übergang vom Studium in den Arbeitsmarkt eröffnen digitale Kommunikations- und Projektmanagementinstrumente einerseits große Chancen, erfordern aber andererseits eine konsequente Weiterentwicklung der bisherigen Kooperationsanstrengungen, auf die das folgende Kapitel eingeht. 


\section{4}

Noch ausbaufähig:

gemeinsame Übergangsgestaltung in die Arbeitswelt 
Eine im Studienjahr 2015 durchgeführte bundesweite Bestandsaufnahme des SVR-Forschungsbereichs (2015) zeigte, dass der damalige Berufseinstieg internationaler Studierender noch zu sehr vom Zufall abhing. Hier und da trafen Bleibewillige an ihrem Hochschulstandort auf ein passendes Bewerbungstraining, ein aufgeschlossenes Unternehmen oder eine serviceorientierte Ausländerbehörde. Insgesamt handelte es sich jedoch meist um kleine und kurzfristig projektfinanzierte Initiativen, die kaum ineinandergriffen und somit nur bedingt zur gewünschten Fachkräftesicherung beitrugen. Vielerorts fehlte es an einer systematischen Zusammenarbeit der zahlreichen lokalen und regionalen Organisationen, die am Übergang vom Studium in den Beruf beteiligt waren und sind. In den Folgejahren wurden Hochschulen dazu ermutigt, Übergänge nicht nur zielgruppengerecht, sondern auch in Kooperation mit externen Partnerorganisationen zu gestalten. ${ }^{25}$ Auch die durch das Bundesministerium für Bildung und Forschung bereitgestellten Fördermittel von Integra und PROFI unterstützen Hochschulen dabei, entsprechende Netzwerkstrukturen auf- und auszubauen. Die bisherige Umsetzung zeugt von einem hohen Maß an Aktivität, verdeutlicht aber gleichzeitig einen zukünftigen Systematisierungsbedarf.

Insgesamt berichten die Projektverantwortlichen an den Hochschulen von mehr als 600 Partnerorganisationen (Integra: 497; PROFI: 137), mit denen sie eine Zusammenarbeit im Zuge der Programmförderung anstreben bzw. bereits pflegen. Durchschnittlich sind dies etwa sieben pro Hochschule. Knapp zwei Drittel der Organisationen sind regional verankert und überwiegend dort tätig. Je nach Schwerpunktsetzung in den Projekten kooperieren die Hochschulen zudem überregional und international. Etwa die Hälfte der Kooperationsbeziehungen ist durch die Projektförderung neu entstanden und befindet sich vielerorts noch im Aufbau. Die andere Hälfte fußt auf bestehenden, zumeist bilateralen Beziehungen (Tab. 6).

Eine vertiefende Auswertung der Kooperationen zeigt, dass Hochschulen mit PROFI-Förderung häufiger mit anderen Wissenschaftseinrichtungen zusammenarbeiten als im Integra-Programm (60 vs. $16 \%$ ). PROFI-geförderte Hochschulen haben zudem eine leicht höhere Tendenz zur Kooperation mit Unternehmen und den Einrichtungen der kommunalen Wirtschaftsförderung, wie etwa den Willkommenszentren (Abb. 3).

Dieser Unterschied dürfte vor allem mit der deutlich berufserfahreneren Zielgruppe von PROFI zusammenhängen, die einen möglichst raschen Einstieg in den hiesigen Arbeitsmarkt anstrebt (s. Kap. 2.2). Deutlich weniger Unterschiede finden sich bei der Zusammenarbeit mit wirtschaftsnahen Mittlerorganisationen, wie etwa der örtlichen Agentur für Arbeit und den regionalen Wirtschaftsverbänden und Kammern. An knapp der Hälfte der Integraund PROFI-geförderten Hochschulen werden

TABELLE 6: PARTNERORGANISATIONEN DER HOCHSCHULEN BEI DER GESTALTUNG DES ÜBERGANGS VOM STUDIUM IN DEN BERUF

$\begin{array}{lrr} & \text { Integra } & \mathbf{1 3 7} \\ \text { Partnerorganisationen } & \mathbf{4 9 7} & 64 \% \\ \begin{array}{l}\text { davon } \\ \quad \text { regional tätig }\end{array} & 67 \% & 24 \% \\ \quad \text { überregional tätig } & 21 \% & 12 \% \\ \quad \text { international tätig } & 12 \% & 45 \% \\ \quad \text { neue Kooperation } & 51 \% & 55 \% \\ \quad \text { bestehende Kooperation } & 49 \% & \end{array}$

25 Siehe z. B. die Empfehlungen des Wissenschaftsrats (2016: 149) und des Bundesamts für Migration und Flüchtlinge (2014: 23) sowie das vom Stifterverband und der Bundesbeauftragten für die neuen Länder geförderte Projekt „Study and Work“ (2015-2017). 
60

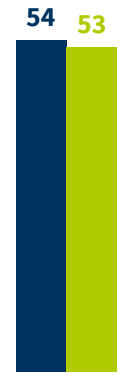

Agentur für Arbeit

Integra
46

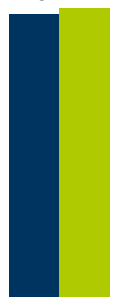

Verbände und Kammern

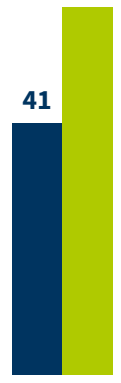

Unternehmen
PROFI

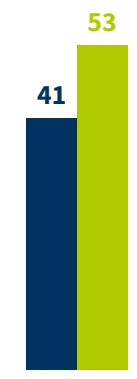

Zivilgesellschaft

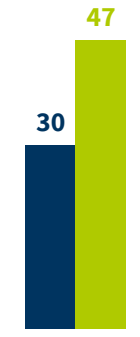

Kommunen
19

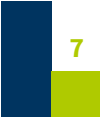

Ausländerbehörde

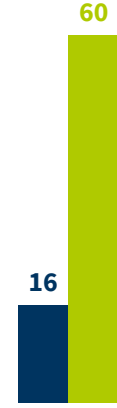

Wissenschaft

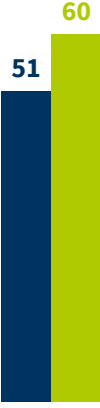

sonstige

Lesehilfe: 54 Prozent der Integra-geförderten Hochschulen und 53 Prozent der Hochschulen mit PROFI-Förderung kooperieren bei der Übergangsgestaltung mit der örtlichen Agentur für Arbeit.

Quelle: Eigene Auswertung der Daten aus Integra und PROFI

diese über Gastvorträge, Workshops und andere übergangsgestaltende Aktivitäten eingebunden (Abb. 3). ${ }^{26}$ Neben der Kooperation mit Externen berichten rund 40 Prozent der Hochschulen von einem sprunghaften Anstieg der hochschulinternen Zusammenarbeit am Übergang vom Studium in den Beruf, z. B. zwischen den Career Services und den Akademischen Auslandsämtern, die an vielen Hochschulen vor Programmbeginn nur sehr sporadisch kooperierten. ${ }^{27}$ Das dort vorhandene zielgruppen- und berufsrelevante Wissen kann somit deutlich effizienter genutzt werden. Insgesamt berichtet das Personal, dass die Zusammenarbeit inner- und außerhalb der Hochschulen in den allermeisten Fällen bilateral und anlassbezogen erfolgt, z. B. im Rahmen von Gastvorträgen oder einer Verweisberatung:

\footnotetext{
Allgemein besteht mit den meisten Kontakten eine bilaterale Zusammenarbeit, die Akteure fungieren als Multiplikatoren und Vermittler für Teilnehmende; es findet ein regelmäßiger Austausch zu angebotenen Maßnahmen und Events statt.

(Mitarbeiter an einer Fachhochschule, PROFI)
}

Die Zusammenarbeit mit der Arbeitsagentur, dem Gründungs- und Innovationszentrum, der Wirtschaftsförderung, dem Hochschulservice und der Krankenkasse erfolgt zunächst bilateral. Die [Partnerorganisationen] vermitteln den am Zertifikatsprogramm teilnehmenden Studierenden wichtiges Wissen, Möglichkeiten und Pflichten zum Berufsstart.

(Mitarbeiterin an einer Universität, PROFI)

Besonders die Workshops zur Bewerbungsvorbereitung in Kooperation mit der Agentur für Arbeit (bilateral) werden von den Studierenden sehr gut angenommen. Außerdem unterstützt das Hochschulteam der Agentur für Arbeit die individuelle Beratung geflüchteter und internationaler Studierender im Rahmen einer wöchentlichen Sprechstunde. (Mitarbeiterin an einer Universität, Integra-Programm)

26 Angesichts der verhältnismäßig kleinen Fallzahl von 88 Hochschulen können die prozentualen Vergleichswerte lediglich Aufschluss über wesentliche Unterschiede zwischen den Kooperationstendenzen in den beiden Hochschulprogrammen geben.

27 Eine im Jahr 2017 unter 119 Career-Service-Einrichtungen durchgeführte Befragung ergab, dass nur 13 Prozent mit den Akademischen Auslandsämtern an ihrer Hochschule zusammenarbeiten (CSND 2018: 12). 
Eine solche bilaterale und anlassbezogene Schwerpunksetzung hat Vorteile: Sowohl die einzelne Hochschule als auch die Partnerorganisation haben dabei ein konkretes Interesse an der Zusammenarbeit. Die Kooperation ist somit von akuter Relevanz und vergleichsweise niedrigschwellig umsetzbar, insbesondere dann, wenn sie die individuellen Stärken der Beteiligten gezielt nutzt. Hier ist erneut das Beispiel der Gastvorträge durch Unternehmen, freiberuflich tätige Bewerbungscoaches sowie auch die Agentur für Arbeit zu nennen. Sie alle werden in viele der untersuchten Projekte regelmäßig eingebunden.

Der Nachteil dieser und weiterer bilateraler Brückenschläge in den Arbeitsmarkt ist, dass vereinzelte Kooperationen zwischen Hochschulen und Unternehmen bzw. sonstigen Partnerorganisationen oft unverbunden nebeneinander stehen und nur einen Teil des gesamten Übergangsgeschehens abbilden (vgl. SVR-Forschungsbereich 2015: 36-39; Roth 2015: 40). Hochschulen, die sich allein auf bilaterale Formen der Zusammenarbeit verlassen, erhöhen somit nicht nur die Gefahr von Doppelstrukturen, auch die Breitenwirkung ihrer Einzelvorhaben ist deutlich geringer als bei multilateralen Kooperationen im Rahmen derer die bilateralen Aktivitäten enger miteinander verzahnt und dauerhafter aufeinander abgestimmt werden. Vielerorts ist der Aufbau multilateraler Netzwerke innerhalb der Projektlaufzeit geplant oder bereits im Gange. Das projektverantwortliche Personal betont jedoch, dass Vernetzung an sich keinen Selbstzweck darstellen sollte und zunächst strategische Richtungsentscheidungen erfordere, vor allem über die Zusammensetzung der Netzwerke. Diese können bspw. je nach Zielsetzung ausgewählte Großunternehmen und Wirtschaftsverbände enthalten, sollten aber den Anschluss an bestehende Netzwerke suchen und das teils konkurrenzbetonte Miteinander vor Ort berücksichtigen:
Ja, es ist jetzt nicht alles heile Welt, [...] sondern es geht um Fachkräfte und Wettbewerb! Wenn man es jetzt mal böse formuliert. Und jeder will ein bisschen was vom seinem Kuchen behalten. Genau, vor dem Hintergrund sondieren wir im Augenblick noch eher das Feld [...]. Wen soll man mit wem auf keinen Fall in Kontakt bringen, weil das wollen die gar nicht? Und mit wem wollen wir wen in Kontakt bringen, weil das total hilfreich ist? Und dann geht es darum, dass wir 2021 jetzt [das Netzwerk] bauen. [...] Mein Endziel ist, lieber klein und fein als groß und nichtssagend. Das ist meine Grunddevise.

(Mitarbeiterin an einer Fachhochschule, PROFI)

Also, wir haben uns 2019 schon Gedanken gemacht, wie können wir diese regionalen Netzwerkpartner für das Thema Übergang in den Beruf bzw. auch Zugang zu Firmen [sensibilisieren]? Also die Universität hat, sozusagen, angefangen so ein Netzwerk aufzubauen, hat sich überlegt, wie könnte das aussehen? Wer sollte auf alle Fälle mit dazu? Da hat uns auf alle Fälle ein bisschen die Geflüchtetenarbeit geholfen in der Region, weil dadurch haben sich sehr viele runde Tische ergeben. Das heißt, man konnte sehr einfach auf verschiedene Pools zugreifen und wir haben jetzt ein Netzwerk, was sich sukzessive vergrößert. [...] Die Genese war, sozusagen, aus [der ersten Förderrunde von] Integra heraus, Weiterentwicklung gibt es ganz stark durch PROFI. [...] Ich glaube, PROFI kann wirklich konkrete Ergebnisse liefern, die für Netzwerkpartnerinnen auch von wichtiger Entscheidung sind, sodass man sagt, ,Okay das Netzwerk bringt auch etwas. Ja, es geht voran.

(Mitarbeiter an einer Universität, PROFI)

Das zweite Zitat hebt die vergleichsweise stärkere Arbeitsmarktorientierung bei den berufserfahreneren Teilnehmenden in den PROFI-Projekten hervor. Diese suchen deutlich früher Kontakt zur regionalen Wirtschaft als ihre Mitstudierenden im Integra-Programm. Das spiegeln auch die laufenden und geplanten Kooperationsaktivitäten in Tab. 7 wieder: 43 Prozent der PROFI-Partner sind 
(perspektivisch) in die konkrete Vermittlung von Arbeitsstellen eingebunden, verglichen mit 7 Prozent im Integra-Programm, in dem es zunächst um Erstkontakte über Unternehmensbesichtigungen, Praxisvorträge, Praktika und studentische Hilfstätigkeiten geht.

Der hohe Anteil an „sonstigen“ übergangsgestaltenden Maßnahmen in den beiden Programmen lässt die Vielfalt der Kooperationsbeziehungen in den geförderten Hochschulprojekten erkennen (31 bzw. 37 \%). Diese umfassen u. a. Hospitationen in Schulen für auslandserfahrene Lehrkräfte, Existenzgründungscoachings für Kunstschaffende und die Einrichtung eines dualen Studiengangs, der insbesondere für internationale Zielgruppen geeignet ist. Entsprechend vielfältig sind die lokalen Kooperationspartner und ihre Vernetzungsbedürfnisse und Zielsetzungen. Diese reichen von mehr Informationsaustausch über eine effizientere, kooperative Abstimmung der jeweiligen Hilfsangebote bis hin zu Bestrebungen der Partner, direkt Einfluss zu nehmen auf konkrete Lehrinhalte an den Hochschulen. Die folgenden Zitate des interviewten Personals in drei Partnerorganisationen veranschaulichen diese Vielfalt und die damit verbundene Notwendigkeit, entlang lokaler Bedürfnisse strategisch zu entscheiden, wer zum Netzwerk passt und wie groß dieses sein sollte (vgl. Innovationsbüro Fachkräfte 2012: 14-16):

\section{[Ich wünsche mir], dass wir noch mehr Netz- werkpartner haben und dass die Informa- tionen in diesem Netzwerk schnell geteilt werden, dass jeder, wenn ich sage, ,Okay, ich brauche Unterstützung, so und so, bei dieser Behörde,' dann kriege ich sofort eine Kontaktperson. Ich denke, das wäre hilfreich auch für uns und auch manchmal für Inter- nationals, wenn wir genau wissen, wohin er oder sie mit dieser Frage hingehen könnte. (Mitarbeiterin in einem regionalen Willkommenszentrum)}

Da [beim Aufbau von Kooperationen] würde ich sagen, da menschelt es halt. Also ich meine, dann glaubt wirklich jemand, alles zu wissen, was natürlich Unsinn ist. Ich denke, ich muss auch letztendlich, ganz gleich wo ich Beratungsdienstleistung anbiete, Kooperationen erkennen, wo sie sinnvoll sind und ich muss vielleicht auch meine Grenzen erkennen und wissen, das kann der andere besser. (Mitarbeiter der Agentur für Arbeit)

\section{Wenn man zum Beispiel Seminare macht} oder Workshops anbietet [...] bevor man solche Seminare macht, man sollte sich mit den Professoren hinsetzen und den Inhalt betrachten, was die Studierenden an den Hochschulen lernen. Weil manche Sachen, ganz ehrlich, sind [in der Arbeitswelt] ausgestorben. Aber sie sind immer noch inhaltlich an der Hochschule.

(Mitarbeiter in einem Unternehmen)

Durch die Kooperationsmaßnahmen konnten an den projektteilnehmenden Hochschulen zwischen Mitte 2020 und Anfang 2021 mehr als 250 Praktika, studentische Jobs und Vollzeitstellen vermittelt werden. Gemessen an den zusammengerechnet 4.425 Projekteilnehmerinnen und teilnehmern in Integra und PROFI ist dieser Wert ausbaufähig, er kann aber zugleich im Lichte der Pandemie als ein ermutigendes Signal interpretiert werden, zumal ein Teil der Projekte zum Beobachtungszeitpunkt aufgrund der Pandemie erst wenige Monate aktiv war. ${ }^{28}$ Viele Unternehmen mussten in der Zwischenzeit auf Kurzarbeit umstellen, Einstellungsstopps verhängen und bereits zugesicherte Praktika absagen. Zudem konnte ein großer Teil der Hochschulen ihre Berufsvorbereitung nur mit mehrmonatiger Verspätung beginnen, was sich ebenfalls auf die Erfolgsbilanz ausgewirkt haben dürfte. Neben der Vermittlung in konkrete Tätigkeiten wurden zudem bislang 160 Vorstellungsgespräche und 18 Abschlussarbeiten in Unternehmen ermöglicht (Tab. 11 im Anhang).

28 Zum Vergleich: 22 Prozent der 5.167 internationalen Studierenden, die im Jahr 2015 an der Studie „Study and Work“ teilgenommen haben, berichteten von einer solchen fachnahen Praxiserfahrung (SVR-Forschungsbereich 2017a). 
TABELLE 7: GEPLANTE UND BEREITS DURCHGEFÜHRTE KOOPERATIONSMASSNAHMEN MIT DEN PARTNERORGANISATIONEN

\begin{tabular}{lrr} 
& PROFI \\
Partnerorganisationen & Integra & $\mathbf{1 3 7}$ \\
davon eingebunden in die Organisation von: & $\mathbf{4 9 7}$ & $19 \%$ \\
$\quad$ Praktika & & $45 \%$ \\
Austauschformate (z. B. runder Tisch) & $18 \%$ & $16 \%$ \\
Bewerbungstrainings & $42 \%$ & $34 \%$ \\
Infoveranstaltungen und Praxisvorträge & $20 \%$ & $43 \%$ \\
Vermittlungsleistungen & $27 \%$ & $3 \%$ \\
Hospitationen & $7 \%$ & $5 \%$ \\
Unternehmensbesichtigungen & $7 \%$ & $1 \%$ \\
Beschäftigung von Werkstudenten & $10 \%$ & $4 \%$ \\
integrierte Praxisphasen & $5 \%$ & $37 \%$ \\
$\quad$ sonstiges & $4 \%$ & $31 \%$ \\
\hline Quelle: Eigene Auswertung der Daten aus Integra und PROFI & &
\end{tabular}

Angesichts der pandemiebedingten Einschränkungen auf den Arbeitsmärkten konzentrierte sich die bisherige Netzwerkarbeit verstärkt auf den Beziehungsaufbau, der i. d. R. sehr zeit- und personalaufwendig ist und primär über virtuelle Kommunikationskanäle realisiert wurde. Bis Anfang 2021 konnte knapp die Hälfte der über 600 Partnerorganisationen über Videokonferenzen und andere virtuelle Austauschformate erreicht werden. In den Fällen, in denen die Organisationen bereits im Vorfeld mit den Hochschulen in Kontakt standen, werden virtuelle Treffen seitens des projektverantwortlichen Personals häufig als effizienter wahrgenommen als die zeitlich und anfahrtstechnisch aufwendigeren Präsenztreffen. Diese Einschätzung teilen auch viele Partnerorganisationen, wie die folgenden Zitate zweier Willkommenszentren verdeutlichen. Ohne einen schon bestehenden Kontakt erweist sich die aufsuchende Netzwerkarbeit und auch die gemeinsame Projektplanung im virtuellen Raum allerdings bisher als sehr mühsam und deutlich weniger ergiebig, nicht zuletzt aufgrund von technischen Problemen: ${ }^{29}$
Also, normalerweise haben wir uns ein, zwei Mal im Jahr in [Name der Stadt] getroffen, aber jetzt online wieder jede zwei, drei Monate sind wir online alle zusammen. Da präsentieren sich neue Mitarbeiterinnen und Mitarbeiter. Da sagt jeder, ,Hey, falls du Unterstützung brauchst...,'[...] Ich habe fünf, sechs Kontakte und ich weiß genau, falls ich Fragen habe, kann ich sie kontaktieren mit den Fragen und die werden mir helfen. Wirklich gut.

(Mitarbeiterin in einem regionalen Willkommenszentrum)

Also im Jahr 2020 im Frühjahr stand [die Zusammenarbeit im Netzwerk] still. Bis August ungefähr. Dann hatten sich alle digital so aufgestellt, dass wir das Ganze wirklich sehr gut auch digital machen können. Das Problem ist natürlich, dass hier jeder seine eigenen Lieblingsmeetings hat. Ja genau, also es gibt Zoom, das dürfen ganz viele nicht verwenden. Das Jobcenter darf es nicht verwenden.

(Mitarbeiterin in einem regionalen

Willkommenszentrum) 


\begin{abstract}
Also, ich muss sagen, mir fällt vieles online auch einfacher, weil es eben schneller geht und man auch direkten Kontakt hat. Bei unseren Unternehmensnetzwerken [...], da haben wir festgestellt, da existiert ein engerer Austausch. [...] Also, wir haben im letzten Jahr auch eine virtuelle Firmenkontaktmesse angeboten, dabei stellt man dann fest, dass sich ein Messeformat etwas schwerer virtualisieren lässt.

(Mitarbeiter an einer Universität, PROFI)
\end{abstract}

Ähnlich wie bei der Digitalisierung der Hochschullehre konzentrieren sich die bisherigen Digitalisierungsanstrengungen auf niedrigschwellige Formate wie virtuelle Arbeitstreffen per Videokonferenz (vgl. Kap 3.1). Wie das dritte Zitat beispielhaft verdeutlicht, wurden geplante Firmenkontaktmessen und andere Großveranstaltungen bislang kaum oder mit nur mäßiger Resonanz in den digitalen Raum verlagert. Der menarbeit im Netzwerk, wie etwa Cloud-Speicher oder Online-Plattformen für ein gemeinsames Projektmanagement, wurde nur vereinzelt erwähnt. Insgesamt sehen die Beteiligten ein großes Weiterentwicklungspotenzial, insbesondere wenn es darum geht, die Stärken traditioneller Präsenztreffen und -veranstaltungen mit den jüngst erfahrenen Vorzügen digitaler Kooperation zu kombinieren.

Die gemeinsame Ausgestaltung einer solch hybriden Netzwerkarbeit braucht ebenso Zeit und personellen Aufwand wie der eigentliche Beziehungsaufbau zu neuen Partnerorganisationen, der vielerorts aufgrund der Pandemie bislang nur sehr langsam vonstattengegangen ist. In den PROFI-Projekten befinden sich 42 Prozent der insgesamt 137 Netzwerkverbindungen noch in einer Anbahnungsphase. Diese Phase dauert nach Auffassung des Hochschulpersonals i. d. R. ein Jahr, wobei die pandemiebedingten Kontaktbeschränkungen viele der grundlegenden persönlichen Erstgespräche deutlich erschweren. Diese sind nach Ansicht des Personals deutlich erfolgreicher, wenn sie von Angesicht zu Angesicht und im bilateralen Austausch über die spezifischen Bedürfnisse der jeweiligen Partnerorganisation erfolgen. Auch das (gemeinsame)

Schaffen von Positivbeispielen wie z. B. Vermittlungserfolge in den regionalen Arbeitsmarkt lassen mancherorts deutlich länger auf sich warten als gehofft. So fällt es mitunter schwer, alte und neue Partnerorganisationen vom Mehrwert einer Netzwerkteilnahme zu überzeugen. Das befragte Hochschulpersonal geht daher mittelfristig von einer verlängerten Aufbauarbeit aus, insbesondere wenn es um multilaterale Beziehungen geht. Die Mittel hierfür stammen bislang fast ausnahmslos aus der Integra- und PROFI-Förderung sowie anderen Drittmittelprojekten. Eine Fortführung über die aktuelle Projektlaufzeit hinaus ist aus Sicht des Personals entscheidend für eine nachhaltige Etablierung tragfähiger Netzwerkstrukturen für den Übergang vom Studium in den Beruf, die es so bislang an kaum einem Standort gibt.

Dass die gemeinsame Übergangsgestaltung bislang fast ausschließlich jeweils bilateral gedacht wird, auch wenn die einzelne Hochschule mit mehreren Partnern kooperiert, verdeutlichen sowohl die obigen Zitate als auch die beobachtete Praxis: Von den insgesamt 137 Partnerorganisationen stehen die PROFI-geförderten Hochschulen bis dato mit 63 Prozent im bilateralen Austausch. Eine gemeinsame Entwicklung von organisationsübergreifenden Strategien und Maßnahmen in einem Netzwerk mehrerer Partner gibt es dagegen nur mit etwa einem Drittel der Partner und auch hier wird nur ein Teil der Aktivitäten multilateral abgestimmt.

Eine stärkere Systematisierung hätte langfristig den Vorteil, dass die gemeinsame Übergangsgestaltung nicht nur deutlich zielgruppenorientierter ausfiele, sondern auch auf finanziell solideren Beinen stünde, insbesondere weil dadurch Reibungsverluste verringert, Doppelstrukturen vermieden und Ressourcen effizienter eingesetzt werden können. Wie ein solches regionales Übergangsmanagement aussehen kann und welche Weiterentwicklungsschritte die Hochschulen zum Aufbau eines solchen gehen können, wird im Schlusskapitel detailliert beschrieben. 


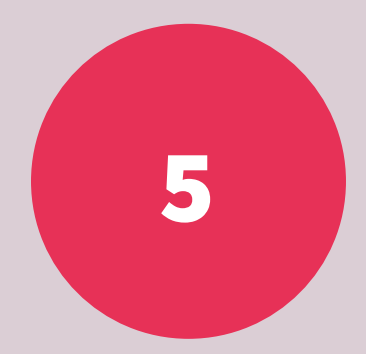

\section{Empfehlungen für Hochschulpraxis und -politik}

5.1 Programme zielgruppengerecht zuschneiden.

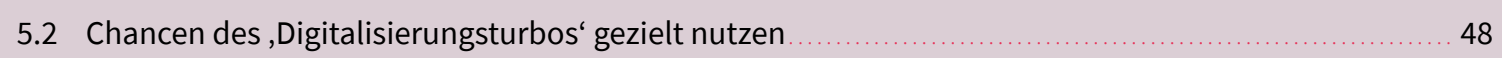

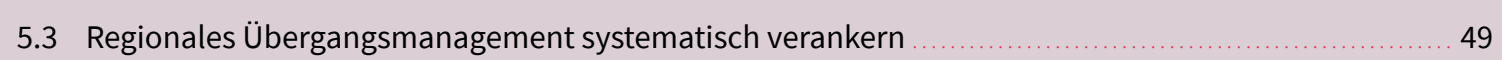

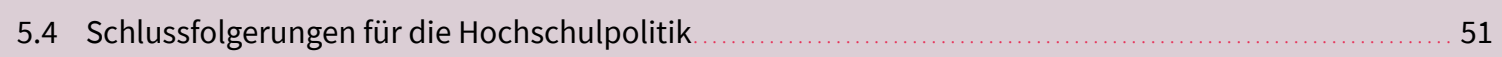


Internationale Studierende werden zunehmend als Idealbesetzung für die wachsende Zahl offener Arbeitsstellen gesehen. Das gilt potenziell auch für die rund 30.000 Geflüchteten, die hierzulande studieren. Dennoch ist ihr Einstieg in den deutschen Arbeitsmarkt kein Selbstläufer. Viele scheitern, weil sie noch nicht perfekt Deutsch sprechen, weil ihnen berufliche Netzwerke fehlen und weil sie zu wenig Arbeitserfahrungen in deutschen Betrieben gesammelt haben. Damit ihr Einstieg in Zukunft besser gelingt, bedarf es nicht nur zusätzlicher Unterstützung, sondern - und das zeigen die Studienergebnisse - eines geschärften Verständnisses der gruppenspezifischen Herausforderungen (s. Kap. 5.1), einer gezielten Weiterentwicklung digitaler Hilfsangebote (s. Kap. 5.2) sowie einer systematischen Wegbereitung von der Studienin die Arbeitswelt in Form eines regionalen Übergangsmanagements (s. Kap. 5.3).

\subsection{Programme zielgruppengerecht zuschneiden}

Insgesamt zeigt die vorliegende Expertise, dass individuelle Fluchtbiografien nicht automatisch zu neuen Herausforderungen beim Übergang vom Hörsaal in den Betrieb führen - sieht man von spezifischen Bedarfen im Bereich der Beratung zum Umgang beispielsweise mit asylrechtlichen Rahmenbedingungen einmal ab (s. Kap. 2.1). Gleichwohl erhöht eine Flucht bei jungen Erwachsenen die bereits bekannten Einstiegsbarrieren. Für die Hochschulen lässt sich daraus schließen, dass zukünftige Fluchtbewegungen keine gesonderte Arbeitsmarktvorbereitung für geflüchtete Studierende erfordern. Allerdings müssen ihre Bedürfnisse stärker als bisher im Regelangebot und punktuell auch in zielgruppenspezifischen Angeboten für internationale Studierende verankert werden. ${ }^{30}$
Eine Anpassung der arbeitsmarktvorbereitenden Regelangebote an die Bedarfslagen internationaler Studierender mit und ohne Fluchterfahrung betrifft insbesondere die breiten Portfolios der hochschulischen Career Services, die neben Bewerbungstrainings, Firmenkontaktmessen und Netzwerkveranstaltungen zunehmend auch Seminare, Unternehmensexkursionen und weitere Angebote enthalten, die auf internationale Zielgruppen zugeschnitten sind und beispielsweise in englischer Sprache durchgeführt werden. Zwar gibt ein großer Teil der Career Services an, bereits Erfahrungen mit der Beratung internationaler Studierender gesammelt zu haben, allerdings fast ausschließlich über projektfinanzierte Vorhaben, die mitunter bereits ausgelaufen sind. Mehr als 60 Prozent der hochschulischen Karriereteams bestehen aus weniger als zwei Vollzeitstellen, wobei nur durchschnittlich eine Stelle aus dem Etat der jeweiligen Hochschule finanziert wird (CSND 2018). Insofern ist die Internationalisierung der Angebote der Career Services bislang noch nicht durchgehend Teil des hochschulischen Regelsystems. Neben den Career Services sind insbesondere die Fakultäten und Fachbereiche für die Entwicklung von fachspezifischen und berufsrelevanten Kompetenzen verantwortlich. Auch hier wird bereits im Lehr- und Betreuungsangebot punktuell auf die vergleichsweise höheren Hürden internationaler Zielgruppen beim Berufseinstieg eingegangen (SVR-Forschungsbereich 2015: 29-31). Angesichts der Vielzahl an Hindernissen, die über den gesamten Studienverlauf auftreten, sollten die Hochschulen ihr Angebot jedoch so ergänzen, dass das Personal in Zukunft stärker auf die Bedarfe internationaler Studierender und die punktuellen Mehrbedarfe Geflüchteter eingehen kann; wichtig ist dabei, dass sie bereits früh im Studium auf den Berufseinstieg vorbereitet werden. Die dahingehende Unterstützung sollte zudem stärker als bisher im Rahmen einer strukturierten Studieneingangsphase umgesetzt werden (SVR-Forschungsbereich 2017: 28-30).

30 Anders bei der Studienvorbereitung: Sowohl die Studierenden als auch das befragte Hochschulpersonal betonten die wegweisende Bedeutung eines möglichst niedrigschwelligen Hochschulzugangs für Geflüchtete, insbesondere für diejenigen, die ihr Studium im Ausland fluchtbedingt unterbrechen mussten. Beim Studieneingang erfordert die besondere Lage vieler Geflüchteter auch in Zukunft eine spezialisierte, individuelle Beratung und Betreuung. 
Neben den Herausforderungen, die sich für internationale Studierende mit und ohne Fluchterfahrung grundsätzlich in ähnlicher Weise stellen, bestehen spezifische Herausforderungen, die sich aus individuellen beruflichen Biografien ergeben, insbesondere bei denjenigen, die im höheren Alter nach Deutschland zuwandern. Hier geht es nicht wie zuvor um eine allgemeine Arbeitsmarktvorbereitung, sondern um eine gezielte Weiterbildung: Diese Personen benötigen ein Lernangebot, das zeitlich komprimiert und präzise abgestimmt ist auf die konkreten Anforderungen in einzelnen Zielberufen und -branchen (s. Kap. 2.2). Dies verdeutlichen die Erfahrungen aus den PROFI-Projekten, deren Pilotierung verschiedener Anpassungskurse einen Vorbildcharakter haben könnte, nicht nur vor dem Hintergrund zukünftiger Fluchtbewegungen in die Bundesrepublik, sondern auch im Zuge der durch das Fachkräfteeinwanderungsgesetz klarer kommunizierten Zugangs- und Bleibemöglichkeiten für Hochqualifizierte: Demnach erhalten Weiterbildungsteilnehmerinnen und -teilnehmer von außerhalb der EU eine Aufenthaltserlaubnis zum Zweck des Studiums (§ 16b Abs. 1 Aufenthaltsgesetz), wenn sie an einer der hiesigen Hochschulen zugelassen sind, sofern sie die geforderten finanziellen, sprachlichen und fachlichen Voraussetzungen nachweisen. Mit dieser Aufenthaltserlaubnis können sie während des Studiums bis zu 120 Tage im Jahr arbeiten (§ 16b Abs. 3 Aufenthaltsgesetz). Wenn sie nach ihrem Abschluss zunächst in Deutschland bleiben und eine Arbeitsstelle suchen möchten, wird ihre Aufenthaltserlaubnis um bis zu 18 Monate verlängert. In dieser Suchphase können sie uneingeschränkt arbeiten (§ 20 Abs. 3 Aufenthaltsgesetz). Ist ihre Suche erfolgreich, können sie bereits nach zwei Jahren Arbeit in Deutschland in einen Daueraufenthalt wechseln (§ 18c Abs. 1 Aufenthaltsgesetz). ${ }^{31}$
Diese Regelungen sind im internationalen Vergleich nicht nur großzügig, sondern ausdrücklich auch für studien- und berufserfahrene internationale Fachkräfte gedacht, die beabsichtigen, sich über eine hochschulische Weiterbildung für den deutschen Arbeitsmarkt zu qualifizieren, z. B. über einen zweisemestrigen Weiterbildungs-Master in internationalem Energierecht oder einen einjährigen Zertifikatskurs in Data Science. ${ }^{32}$ Allerdings sind kürzere, angewandte und auf internationale Fachkräfte zugeschnittene Lernangebote wie diese bislang noch rar gesät. Die Hochschulen sollten daher erwägen, ihr oftmals noch sehr überschaubares Angebot an berufsbegleitenden Studiengängen und (modularisierten) Zertifikatskursen stärker für internationale Zielgruppen zu öffnen. Dies könnte auch über Verbundprojekte zwischen mehreren Hochschulen geschehen (vgl. Wissenschaftsrat 2019: 74-85; Borgwardt 2016: 7-8). Dabei sollten die in PROFI gemachten Erfahrungen mit den sprachlichen, arbeitskulturellen und strukturellen Hürden, vor denen viele Kursteilnehmende stehen, gezielt berücksichtigt werden. Die Entwicklung dieser Weiterbildungsangebote könnte über eine Mischfinanzierung aus staatlichen und privatwirtschaftlichen Mitteln erfolgen. Auch die Lernenden sollten sich an den Kosten beteiligen. Dort wo ein hohes gesellschaftliches Interesse an einer frei zugänglichen Weiterbildung besteht, wie etwa bei der Arbeitsmarktintegration von Geflüchteten, sollten Teilnahmegebühren allerdings entfallen oder durch Stipendien getragen werden (vgl. Wissenschaftsrat 2019: 67-71).

Das Potenzial einer solchen Internationalisierung ist hoch: In Kanada waren 2019 knapp 90.000 internationale Studierende in ein- bis zweijährigen praxisorientierten Bildungsprogrammen eingeschrieben. Insbesondere die für berufserfahrene Zielgruppen konzipierten Zertifikatskurse (post-graduate certificates) locken zunehmend Fachkräfte aus dem Ausland, die sich neben fachlichem Input auch

31 Grundvoraussetzung ist, dass die Teilnehmenden einen „anerkannten höheren Bildungsabschluss“ an einer Hochschule erwerben, zu denen neben einem Weiterbildungs-Master z. B. auch ein Hochschulzertifikat zählt, dem ein ausländisches Erststudium vorausgeht (Lehner 2019: 131-134; Berliner Landesamt für Einwanderung 2021: 128-129).

32 Neben diesen beiden Programmen enthält die DAAD-Datenbank international ausgerichteter Studienangebote derzeit 250 MasterStudiengänge mit kürzeren Laufzeiten von drei und teilweise auch zwei Semestern, sowie eine Handvoll Kurzprogramme (https://www2.daad. de/deutschland/studienangebote/international-programmes/en/, 27.05.2021). 
einen beschleunigten Zugang zum kanadischen Arbeitsmarkt versprechen. ${ }^{33}$ Die hiesigen Hochschulen sollten daher die Möglichkeit prüfen, ihr Weiterbildungsangebot mit Blick auf internationale Zielgruppen weiterzuentwickeln. Hier ist beispielsweise auch an Personen zu denken, die im Rahmen des Familiennachzugs mit Partnern oder Partnerinnen nach Deutschland kommen, die hier eine Erwerbstätigkeit aufnehmen und die häufig zunächst nicht oder unter ihrer Qualifikation beschäftigt sind.34 Der Qualifizierungsbedarf von Geflüchteten insbesondere derjenigen, die ihr voriges Studium noch nicht zu Ende bringen konnten - sollte dabei stets mitgedacht werden. Ob und inwiefern Bund und Länder die Hochschulen hierbei unterstützen, sollte nicht zuletzt vor dem Hintergrund der darin liegenden Potenziale zur Fachkräftegewinnung erwogen werden.

\subsection{Chancen des, Digitalisierungs- turbos' gezielt nutzen}

Die kurzfristig erfolgte Digitalisierung der arbeitsmarktvorbereitenden Angebote in den Integra- und PROFI-Projekten hat Geflüchtete und andere internationale Studierende vor neue Herausforderungen gestellt. Dennoch zeugen die bisherigen Erfahrungen von einer grundsätzlichen Offenheit gegenüber digitalen und hybriden Formen der Arbeitsmarktvorbereitung (s. Kap. 3). Diese Offenheit sollte auch nach Ende der pandemiebedingten Kontaktbeschränkungen gezielt genutzt werden, nicht zuletzt, weil der Berufseinstieg internationaler Zielgruppen eine betreuungsintensive Aufgabe ist und digitale Technologien wie z. B. interaktive Videokonferenzen hier punktuell Abhilfe schaffen und eine Skalierung von Angeboten ermöglichen können. Damit diese und andere Innovationen in der Hochschulpraxis Fuß fassen können, bedarf es nicht nur eines gezielten Auf- und Ausbaus der digitalen
Infrastruktur und Kompetenzen - sowohl bei den Lernenden als auch beim Personal -, sondern auch einer didaktischen Anpassung der gesamten digitalen Angebotspalette an die Erfordernisse des Lernens am Bildschirm.

Im Vergleich zu ihren deutschen Studienkolleginnen und -kollegen verfügen Geflüchtete und andere internationale Studierende nicht immer über die notwendigen Endgeräte oder eine schnelle Internetverbindung. Bund, Länder und die Hochschulen sollten daher mehr tun, um die Bedürftigen unter ihnen bei der Beschaffung der entsprechenden Technik und Zugänge zu unterstützen. Angesichts der auch auf lange Sicht großen Bedeutung von Online-Lehre sollte eine solche Unterstützungsleistung langfristig verankert sein, z. B. über eine entsprechende Erweiterung des Bundesausbildungsförderungsgesetzes (BAFöG) (vgl. Stifterverband 2020: 13).

Technik allein wird jedoch nicht automatisch zu den gewünschten Erfolgen im Studium und beim Berufseinstieg führen. Lernende und Lehrende benötigen mehr Zeit und Training, um im virtuellen Kursgeschehen kompetent agieren $\mathrm{zu}$ können. Neben der technischen Einarbeitung ist auf Seiten der internationalen Zielgruppen mehr Unterstützung in den Bereichen OnlineKommunikation, selbstorganisiertes Lernen und dem reflektierten Umgang mit digitalen Medien erforderlich. Für das Hochschulpersonal besteht neben dem Auf- und Ausbau technischer Fertigkeiten ein Qualifizierungsbedarf insbesondere in der didaktischen Professionalisierung für den digitalen Unterricht in einem sprachlich und lernkulturell vielfältigen Umfeld (vgl. Hochschulforum Digitalisierung 2020: 24-28; Seyfeli/Elsner/Wannemacher 2020: 7).

Jenseits dieser individuellen Lehrkompetenzen bedarf es zudem breit angelegter hochschuldidaktischer Anpassungen: Da die

33 Gemäß der Internationalen Standardklassifizierung im Bildungswesen (ISCED) waren in Kanada im Jahr 2019 insgesamt 88.683 internationale Studierende in kurzen tertiären Bildungsprogrammen der ISCED-Stufe 5 eingeschrieben. Hierzu zählen neben den genannten Zertifikatskursen auch die sog. advanced diplomas sowie auch undergraduate diplomas, für die kein voriger Hochschulabschluss benötigt wird (Statistics Canada 2021).

34 Das Fachkräftepotenzial ist an dieser Stelle besonders hoch: Zwischen 2005 und 2017 sind schätzungsweise 800.000 ausländische Partnerinnen und Partner nachgezogen - rund drei Viertel von ihnen sind Frauen, von denen ein Drittel ein Studium absolviert hat und ein weiteres Drittel studienberechtigt ist. Dennoch sind 56 Prozent der nachgereisten Partnerinnen nicht erwerbstätig, obwohl viele es sich wünschen (Borowsky et al. 2020: 25-32). 
,Expressdigitalisierung' der Arbeitsmarktvorbereitung in den Integra- und PROFI-Projekten bislang eher auf das kurzfristig Machbare abzielte und weniger auf den tatsächlichen didaktischen Bedarf einzelner Studierendengruppen, erscheint eine dahingehende Weiterentwicklung notwendig, damit auch Geflüchtete und andere Studierende ohne muttersprachliche Deutschund Englischkenntnisse voll profitieren können (vgl. Stifterverband 2020: 15-17). Hierfür sollten die derzeitigen Ad-hoc-Maßnahmen von den jeweiligen Hochschulen zunächst evaluiert werden. Bewährte Ansätze könnten dann in einen reformierten Regelbetrieb überführt werden, in dem Präsenzphasen und digitale Lernkomponenten einander ergänzen und die jeweiligen Stärken gezielt genutzt werden. Auf der Suche nach der idealen Mischung aus Online- und Präsenzlehre sollten sowohl das Lehrpersonal als auch die Studierenden kontinuierlich einbezogen werden (Stifterverband 2020: 10). Auch Unternehmen und andere außerhochschulische Partnerorganisationen sollten hier Mitgestaltungsmöglichkeiten erhalten, z. B. bei der Entwicklung virtueller und hybrider Praxiserfahrungen, die bis dato an nur wenigen Hochschulstandorten etabliert sind. Ein interessantes Beispiel hierfür findet sich in Kanada, wo das Bildungsunternehmen Riipen echte Firmenaufträge an internationale Studierende vermittelt. Auf einem digitalen Marktplatz können die Betriebe aus einer Liste praxisrelevanter Studienprojekte das für sie passende auswählen und eigene Arbeitsaufträge vergeben. Die Studierenden bearbeiten diese anschließend und erwerben somit frühzeitig und ortsunabhängig arbeitsmarktrelevante Kompetenzen und wertvolle Kontakte für ihren späteren Berufseinstieg. ${ }^{35}$

Derzeit wird ein Großteil der digitalen Umstrukturierung inner- und außerhalb der Hochschulprojekte über zeitlich begrenzte Projektmittel finanziert. In einer Studien- und Arbeitswelt, in der hybride Lern- und Beschäftigungsszenarien zunehmend zum Normalfall werden, sollte die Digitalisierung stärker als bisher als eine dauerhafte Querschnittsaufgabe wahrgenommen und entsprechend grundfinanziert werden.

\subsection{Regionales Übergangs- management systematisch verankern}

Die Erfahrungen in den Integra- und PROFIgeförderten Hochschulprojekten verdeutlichen, dass eine auf internationale Zielgruppen zugeschnittene Gestaltung des Übergangs vom Studium in den Arbeitsmarkt bisher in den allermeisten Fällen bilateral und anlassbezogen erfolgt, z. B. im Rahmen eines Gastvortrags durch ein Partnerunternehmen oder eines Verweises auf das Beratungsangebot der örtlichen Agentur für Arbeit (s. Kap. 4). Vereinzelte, bilaterale Verbindungen in die Arbeitswelt sind jedoch nicht genug, um geflüchtete und andere internationale Studierende beim Berufseinstieg zu begleiten. Dies erfordert vielmehr ein regionales Übergangsmanagement, das die zahlreichen Einzelinitiativen von Hochschulen, Unternehmen, kommunaler Wirtschaftsförderung, Arbeitsvermittlung sowie weiterer regionaler Partnerorganisationen miteinander verzahnt und so den internationalen Fachkräften von morgen klare Wege in den (regionalen) Arbeitsmarkt aufzeigt (SVRForschungsbereich 2015: 45-46; 2019: 40-41). Von einem solchen multilateralen Brückenschlag zwischen Studien- und Arbeitswelt können auch deutsche Studierende profitieren.

Die im Rahmen von Integra und PROFI geschlossenen Kooperationsvereinbarungen mit mehr als 600 Partnerorganisationen sind ein fruchtbarer Boden für den Aufbau eines regionalen Übergangsmanagements. Hierfür sollten die zahlreichen bilateralen und anlassbezogenen Kooperationen weiter gestärkt und systematisch entlang des regionalen Fachkräftebedarfs miteinander vernetzt werden. Obwohl sich solche multilateralen Netzwerke letztendlich von Ort zu Ort unterschiedlich zusammensetzen, sind die Schritte dorthin i. d. R. die gleichen:

- Transparenz schaffen und weitere Partnerorganisationen identifizieren: Für eine Zusammenarbeit sind neben den aktuellen Kooperationspartnern zunächst weitere Organisationen 
vor Ort auszumachen, die beim Übergang vom Studium in den Beruf eine Rolle spielen bzw. spielen könnten. Über bi- und multilaterale Gespräche sollte dann erfasst werden, welche Einstellungen und Ziele sie jeweils im Hinblick auf den Berufseinstieg internationaler Fachkräfte haben und was sie bereits dafür tun. Unternehmen, Behörden und Mittlerorganisationen, die in dieser Hinsicht noch passiv sind, werden somit dafür sensibilisiert, dass auch an den örtlichen Hochschulen internationale Fachkräfte gewonnen werden können. Aufbauend auf diesen Erstgesprächen sollte strategisch entschieden werden, wer zum Netzwerk passt, wer nicht und wie groß dieses zunächst sein soll. Für den Anfang kann es zielführend sein, die Zahl der Partnerorganisationen zu begrenzen (vgl. f-bb/ isw 2018: 15-17; SVR-Forschungsbereich 2015: 45; 2019: 40).

- Gemeinsame Ziele und Regeln entwickeln: Für das regionale Übergangsmanagement sollten erreichbare Ziele gesteckt und dafür entsprechende Maßnahmen und messbare Indikatoren bestimmt und schriftlich festgehalten werden. Darüber hinaus sollten die Partnerorganisationen gemeinsame Regeln erarbeiten, um etwaige interne Konflikte zu lösen. Diese werden ebenfalls schriftlich niedergelegt (Innovationsbüro Fachkräfte 2012: 14-16; SVR-Forschungsbereich 2015: 45; 2019: 40).

- Netzwerkstrukturen einrichten und festigen: Als Grundlage für die operative Umsetzung der strategischen Ziele sollte ein „Runder-TischÜbergangsmanagement" oder ein anderes Kommunikationsformat eingerichtet oder ein Format aus bestehenden regionalen Fachkräfteinitiativen genutzt werden: Hier treffen die Partnerorganisationen mindestens einmal pro Quartal zusammen, um ihre Unterstützungsangebote aufeinander abzustimmen und neue Ziele festzulegen. Neben gelegentlichen Präsenztreffen sollten insbesondere auch Videokonferenzen, Online-Projektmanagementplattformen und weitere digitale Instrumente je nach Bedarf zum Einsatz kommen. Ein erfolgreiches Übergangsmanagement lebt von Vertrauen und stetigem Austausch. Darum sollten alle Beteiligten feste Ansprechpersonen benennen und diese für die Teilnahme an den Steuerungsrunden freistellen.
Auch (ehemalige) internationale Studierende mit und ohne Fluchterfahrung sollten einbezogen werden (vgl. f-bb/isw 2018: 21-23; SVR-Forschungsbereich 2015: 45; 2019: 40).

- Mehrwert erfahrbar machen: Wichtig ist, dass die Beteiligten nicht in einem ,Silo-denken“ verharren, das nach wie vor weit verbreitet ist. Darum müssen Hochschulen, Kommunen und insbesondere die Unternehmen regelmäßig vom Nutzen ihres Engagements überzeugt werden. Solche Überzeugungsarbeit können öffentlich wirksame Leuchtturmprojekte leisten, etwa eine jährlich stattfindende regionale Karrieremesse für internationale Studierende. In einigen der untersuchten Hochschulprojekte wurden solche Veranstaltungen zwar geplant, mussten aber aufgrund der Pandemie entweder vertagt, abgesagt oder digital durchgeführt werden, wobei auch Letzteres nicht immer den gewünschten Vernetzungseffekt mit sich brachte. Trotz oder gerade wegen dieser Erfahrungen sollten innovative Konzepte der gemeinsamen digitalen und hybriden Übergangsgestaltung zukünftig stärker gefördert werden, wie etwa ein hybrides Reverse-Mentoring, bei dem internationale Studierende das Personal in regionalen Betrieben mittels Videokonferenzen und Präsenztreffen beispielsweise über potenzielle Absatzmärkte im arabischen Raum, Fernost oder anderen Erdteilen informieren und im Gegenzug wertvolle Einblicke in die Praxis sowie gegebenenfalls einen Praktikumsplatz erhalten. Hier könnten u. a. auch die Kammern und die Agentur für Arbeit als Türöffner agieren (vgl. f-bb/isw 2018: 25-27; Innovationsbüro Fachkräfte 2012: 18; SVR-Forschungsbereich 2015: 46; 2019: 41).

Damit ein regionales Übergangsmanagement nicht nur auf dem Papier besteht, muss die Zusammenarbeit der Partnerorganisationen verlässlich organisiert werden. Dafür bedarf es der gezielten Koordination, sonst werden Arbeitstreffen $u$. U. nicht hinreichend vorbereitet, sind schlecht besucht oder fallen ganz aus. Zudem muss es eine feste Ansprechperson geben, die die Beteiligten insgesamt nach außen vertritt (z. B. gegenüber der Presse) und auch intern bei Konflikten vermittelt. Mithilfe der Förderung aus Integra und PROFI haben 88 Hochschulen hier bereits die Initiative ergriffen. Darauf sollte 
nun aufgebaut werden. Damit ihre bi- und multilaterale Netzwerkarbeit auch nach Projektende fortgeführt und perspektivisch aus Mitteln der Hochschulen und ihrer Partnerorganisationen getragen werden kann, bedarf es vielerorts einer zusätzlichen Aufbau- und Überzeugungsarbeit. Hier erscheint es lohnenswert, die nun geschaffenen Strukturen und Kooperationsbeziehungen weiterzuentwickeln.

\subsection{Schlussfolgerungen für die Hochschulpolitik}

Die Fachkräftemigration sowie die Flüchtlingsintegration über die Hochschulen sind ein erklärtes Ziel von Politik und Wirtschaft. Und auch die Hochschulen selbst zeigen ein reges Interesse, insbesondere die Career Services und die Akademischen Auslandsämter. Andererseits basiert ihr bisheriger Beitrag zur Fachkräftesicherung nur auf kürzeren und i. d. R. projektfinanzierten Pilotvorhaben. Die Praxis zeigt, dass dort, wo Projektmittel bereits ausgeschöpft sind, das Erarbeitete zwar in den Regelangeboten punktuell ,mitgedacht' wird. Die Unterstützung auf dem vorherigen Niveau fortzuführen, ist aber kaum möglich. Zwar sieht das im Rahmen von Integra und PROFI befragte Hochschulpersonal grundsätzlich die Möglichkeit, mittelfristig eine nachhaltige Finanzierung seitens ihrer Hochschule oder der regionalen Wirtschaft zu sichern. Hierfür muss die nun angestoßene Arbeitsmarktintegration jedoch über mehrere Jahre engagiert weiterverfolgt, systematisch dokumentiert und wirksam kommuniziert werden, sowohl regional als auch überregional.
Damit eine solche Verstetigung in der Fläche gelingen kann, bedarf es einer gezielten und zeitlich begrenzten Förderung, die sich an den bisherigen Erkenntnissen zum Berufseinstieg der verschiedenen internationalen Zielgruppen orientiert. Die vorliegende Begleitstudie verdeutlicht, dass die hohen Einstiegshürden zum deutschen Arbeitsmarkt für Geflüchtete und andere internationale Zielgruppen an den hiesigen Hochschulen eine frühzeitig ansetzende, hybride Berufsvorbereitung erfordern, die sich verstärkt an individuellen Bildungs- und Berufsbiografien orientiert und Übergänge gemeinsam gestaltet. Die bundesweite Förderung eines solchen regionalen Übergangsmanagements könnte entscheidend dazu beitragen, die oft noch vage Rolle der Hochschulen bei der Fachkräftemigration zu präzisieren und somit den Verbleib der begehrten Talente zu unterstützen. Ziel der Förderung sollte zudem eine nachhaltige finanzielle Beteiligung der Hochschulen und der regionalen Wirtschaft am Brücken- 


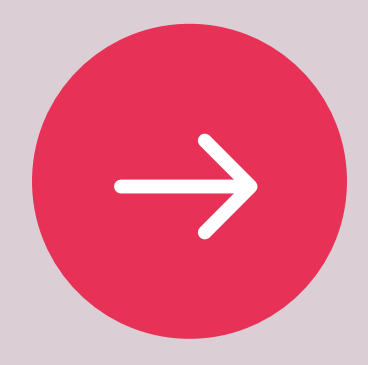

\section{Anhang}

A Literaturverzeichnis 


\section{A Literaturverzeichnis}

Apolinarski, Beate/Brandt, Tasso 2018: Ausländische Studierende in Deutschland 2016, Berlin.

Arajärvi, Outi/Drubig, Roland 2014: VISS - Verbleibspotenzial internationaler Studierender in Sachsen. Abschlussbericht mit Handlungsempfehlungen, Leipzig.

BAMF - Bundesamt für Migration und Flüchtlinge 2014: Interkulturelle Öffnung von Hochschulen, Nürnberg.

BAMF 2016: Hochschulzugang und Studium von Flüchtlingen. Eine Handreichung für Hochschulen und Studentenwerke, Nürnberg.

Bayerischer Landtag 2020: Antrag der Abgeordneten Thomas Kreuzer et al. Reform des Hochschulgesetzes: Deutschkenntnisse und Mehrsprachigkeit der Studierenden fördern! Drucksache 18/6704, München.

BBSR - Bundesinstitut für Bau-, Stadt- und Raumforschung 2018: Raumordnungsbericht 2017: Daseinsvorsorge sichern, Bonn.

BDA/HRK/BDI - Bundesvereinigung der Deutschen Arbeitgeberverbände/Hochschulrektorenkonferenz/Bundesverband der Deutschen Industrie 2014: Internationales Potenzial für Deutschlands Zukunft nutzen. Kompetenzen für Wissenschaft und Wirtschaft gewinnen, Berlin.

Berliner Landesamt für Einwanderung 2021: Verfahrenshinweise zum Aufenthalt, Berlin.

BIBB - Bundesinstitut für Berufsbildung 2021: Auswertung der amtlichen Statistik zum Anerkennungsgesetz des Bundes für 2020. Ergebnisse des BIBB-Anerkennungsmonitorings, Bonn.

Bitkom 2020: Homeoffice für alle? Wie Corona die Arbeitswelt verändert. Präsentation vom 08.12.2020. (https://www.bitkom.org/sites/default/files/2020-12/bitkom-charts-homeoffice-08-12-2020_final_0.pdf, 02.09.2021)

BMAS - Bundesministerium für Arbeit und Soziales 2011: Fachkräftesicherung. Ziele und Maßnahmen der Bundesregierung, Berlin.
BMI - Bundesministerium des Innern 2012: Jedes Alter zählt. Demografiestrategie der Bundesregierung, Berlin.

BMBF - Bundesministerium für Bildung und Forschung 2017: Internationalisierung von Bildung, Wissenschaft und Forschung. Strategie der Bundesregierung, Bonn.

Borgwardt, Angela 2016: Akademische Weiterbildung. Friedrich Ebert Stiftung. Schriftenreihe Hochschulpolitik, Berlin.

Borowsky, Christine/Schiefer, David/Neuhauser, Bastian/Düvell, Franck 2020: Erwerbskräftepotenzial von Personen im partnerschaftlichen Familiennachzug aus dem EU-Ausland und aus Drittstaaten. DeZIM Project Report 2/20, Berlin.

Brandl, Heike 2007: Schwierigkeiten beim Verfassen akademischer Texte in der Fremdsprache Deutsch. Schreibdidaktische Konsequenzen für Studienbegleitung und -vorbereitung, in: Beiträge zur chinesisch-deutschen Fachkonferenz" Aspekte der Studienvorbereitung/Studienbegleitung" vom 21./22. Mai 2007 am Deutschkolleg der TongjiUniversität Shanghai, 165-182.

Bridge Netzwerk - Bridge Berliner Netzwerke für Bleiberecht 2021: Geflüchtete: Arbeitsmarktzugang und -förderung. Ein Leitfaden für Mitarbeitende von Arbeitsagentur und Jobcenter, Berlin.

Brücker, Herbert/Kunert, Astrid/Mangold, Ulrike/Kalusche, Barbara/Siegert, Manuel/Schupp, Jürgen 2016: Geflüchtete Menschen in Deutschland. Eine qualitative Befragung. IAB-Forschungsbericht 9/2016, Nürnberg.

Brücker, Herbert/Croisier, Johannes/Kosyakova, Yuliya/Kröger, Hannes/Pietrantuono, Giuseppe/Rother, Nina/Schupp, Jürgen 2019: Zweite Welle der IAB-BAMF-SOEP-Befragung: Geflüchtete machen Fortschritte bei Sprache und Beschäftigung. IAB-Kurzbericht 3/2019, Nürnberg. 
Brücker, Herbert/Kosyakova, Yuliya/Schuß, Eric 2020: Fünf Jahre seit der Fluchtmigration 2015. Integration in Arbeitsmarkt und Bildungssystem macht weitere Fortschritte. IAB-Kurzbericht 04/2020, Nürnberg.

Burstedde, Alexander/Hickmann, Helen/Schirner, Sebastian/Werner, Dirk 2021: Ohne Zuwanderung sinkt das Arbeitskräftepotenzial schon heute. Mehr Renteneintritte als Nachwuchs-Arbeitskräfte überall. IW-Report 25/2021, Köln.

\section{CSND - Career Service Netzwerk Deutschland}

2018: Ausgangslage der Career Services in Deutschland 2017, Berlin.

DAAD - Deutscher Akademischer Austauschdienst

2014a: Qualifizierte internationale Bewerber gewinnen und Studienerfolg sichern. Vorschläge für eine Reform des Hochschulzugangs für Ausländer, Bonn.

DAAD 2014b: Ergebnisbericht zur Evaluierung des DAAD-Programms - STIBET I und STIBET III Matching Funds, Berlin.

DAAD 2017: Integration von Flüchtlingen an deutschen Hochschulen. Erkenntnisse aus den Hochschulprogrammen für Flüchtlinge. Information 1 Studienvorbereitung und Studienzugang, Köln.

DAAD 2018: Integration von Flüchtlingen an deutschen Hochschulen. Erkenntnisse aus den Hochschulprogrammen für Flüchtlinge. Information 2 Studienvorbereitung und Übergang ins Studium, Köln.

DAAD 2020a: Integration von Flüchtlingen an deutschen Hochschulen. Erkenntnisse aus den Hochschulprogrammen für Flüchtlinge. Information 3 Einstieg ins Fachstudium und Studienbegleitung, Köln.

DAAD 2020b: COVID-19 and the Impact on International Student Mobility in Germany. Results of a DAAD Survey Conducted among International Offices of German Universities. DAAD Working Paper, Bonn.

DAAD/DZHW 2021: Wissenschaft Weltoffen 2020 Excel Tabellen. (http://www.wissenschaftweltoffen. de/wwo2020/, 02.09.2021)

DZHW - Deutsches Zentrum für Hochschul- und Wissenschaftsforschung 2020: Studieren unter Corona-Bedingungen: Studierende bewerten das erste Digitalsemester. DZHW-Brief 5/2020, Hannover.
DZHW 2021a: Studieren in Zeiten der CoronaPandemie: Vulnerable Studierendengruppen im Fokus. DZHW-Brief 2/2021, Hannover.

DZHW 2021b: Die Studiensituation in der CoronaPandemie. Auswirkungen der Digitalisierung auf die Lern- und Kontaktsituation von Studierenden. DZHW-Brief 1/2021, Hannover.

Damelang, Andreas/Kosyakova, Yuliya 2021: To Work or to Study? Postmigration Educational Investments of Adult Refugees in Germany - Evidence from a Choice Experiment, Research in Social Stratification and Mobility, 73, June 2021, 1-12.

Dömling, Martina 2013: Willkommenssignale setzen. Ausländische Studierende in Ostdeutschland halten, in: Pasternack, Peer (Hrsg.): Jenseits der Metropolen. Hochschulen in demografisch herausgeforderten Regionen, Leipzig, 470-496.

Engel, Ole/Wolter, Andrä 2017: Flüchtlinge als Zielgruppe wissenschaftlicher Weiterbildung?, in: Hochschule und Weiterbildung, 1/2017, 63-74.

Ekert, Stefan/Wallau, Frank/Werner, Jennifer/ Will, Anne-Kathrin 2014: Werdegang internationaler Fachkräfte und ihr Mehrwert für KMU, Berlin.

Erdsiek, Daniel/Elbert, Sabine 2021: Homeoffice nach Corona: Erwartete Nutzung steigt weiter. Unternehmensbefragung in Informationswirtschaft und Verarbeitendem Gewerbe, Pressemitteilung vom 04.08.2021. (https://www.zew.de/PM7847, 02.09.2021)

Fachkommission Integrationsfähigkeit Fachkommission der Bundesregierung zu den Rahmenbedingungen der Integrationsfähigkeit 2020: Gemeinsam die Einwanderungsgesellschaft gestalten, Frankfurt am Main.

\section{f-bb/isw - Forschungsinstitut Betriebliche} Bildung/Institut für Strukturpolitik und Wirtschaftsförderung 2018: Praxishilfe. Zielführende Kooperation in Netzwerken der Region, Halle/Saale.

Fintiba 2020: How Has the Coronavirus Affected the (Study) Plans of International Students? Fintiba Insights 1/2020, Frankfurt am Main. 
Gödersmann, Udo 2021: Der BAföG Anspruch nach Fachrichtungswechseln oder Abbruch der Ausbildung. Umfassende Darstellung der rechtlichen Grundlagen nebst praktischen Hinweisen zum Aufbau einer Begründung. Mit Beispielen und Musterschreiben. AStA Sozialberatung an der Uni Duisburg-Essen. (https://asta-due.de/wp-content/ uploads/2021/05/BAfoeG-und-Fachrichtungswechsel-5-2021.pdf, 13.09.2021)

Goertz, Lutz/Hense, Julia 2021: Studie zu Veränderungsprozessen in Unterstützungsstrukturen für Lehre an deutschen Hochschulen in der CoronaKrise, Arbeitspapier Nr. 56 des Hochschulforums Digitalisierung, Berlin.

Grüttner, Michael/Schröder, Stefanie/Berg, Jana 2020: Erfolgserwartung und Abbruchneigung bei internationalen Studieninteressierten und Geflüchteten in der Studienvorbereitung, in: JungbauerGans, Monika/Gottburgsen, Anja (Hrsg.): Migration, Mobilität und soziale Ungleichheit in der Hochschulbildung, Wiesbaden, $169-196$.

Hanganu, Elisa/Heß, Barbara 2014: Beschäftigung ausländischer Absolventen deutscher Hochschulen. Ergebnisse der BAMF-Absolventenstudie 2013, Nürnberg.

Heublein, Ulrich/Richter, Johanna/Schmelzer, Robert 2020: Die Entwicklung der Studienabbruchquoten an den deutschen Hochschulen, Hannover.

HFD - Hochschulforum Digitalisierung 2020: KurZ \& kompakt. Das digitale Sommersemester 2020: Was sagt die Forschung?, Berlin.

Innovationsbüro Fachkräfte für die Region 2012: Gute Praxis - Ideen und Anregungen zur Fachkräftesicherung in der Region, Berlin.

Jayadeva, Sazana 2020: The Impact of Covid-19 on Postgraduate-Level Student Migration from India to Germany. CHGE Research Finding No. 6, Oxford.

Jacob, Anna Katharina/Hermann, Julia/Schmidt, Eva 2014: Nachqualifizierung für ausländische Akademikerinnen und Akademiker: Erste Erfahrungen aus dem Projekt ProSALAMANDER an der Universität Duisburg-Essen, in: Düll, Nicola/von Helmolt, Katharina/Prieto Peral, Begoña/Rappenglück, Stefan/ Thurau, Lena (Hrsg.): Migration und Hochschule. Herausforderungen für Politik und Bildung, Stuttgart, 95-112.
Klabunde, Niels 2014: Wettlauf um internationale Studierende. Integration und interkulturelle Hochschulentwicklung in Deutschland und Kanada, Wiesbaden.

Knuth, Matthias 2020: Ausweg aus der Förderungsfalle. Studium in einem anderen Fach nach fluchtbedingtem Studienabbruch - Verwaltungsgericht findet neuen Ansatz, in: Soziale Sicherheit 2020: 10, 367-370.

Kratz, Fabian/Reimer, Maike 2013: Frühe Bindung zahlt sich aus: Wie Arbeitgeber und Studierende von frühzeitigen Kontakten profitieren, München.

Kruse, Jan 2014: Qualitative Interviewforschung. Ein integrativer Ansatz, Weinheim/Basel.

Ladwig, Tina 2019: Open for all. Learning Management-Systems und OER in der Hochschullehre. (https://hochschulforumdigitalisierung.de/de/blog/ open-for-all, 02.09.2021)

Lancee, Bram 2019: Ethnic Discrimination in $\mathrm{Hi}$ ring: Comparing Groups across Contexts. Results from a Cross-National Field Experiment, in: Journal of Ethnic and Migration Studies. (https://doi.org/10. 1080/1369183X.2019.1622744, 24.08.2021)

Lehner, Roman 2019: Bildungsmigrationsrecht in Deutschland: Systematik, Zielsetzung und Entwicklung, in: Recht der Jugend und des Bildungswesens, 67: 2, 126-141.

Lörz, Markus 2020: Corona Pandemie, veränderte Studienbedingungen und soziale Ungleichheiten im Studium? (https://www.studentenwerke.de/sites/ default/files/views_filebrowser/v1_vortrag_loerz_ dsw_sfs_2021.pdf, 02.09.2021)

Meschter, Diana/Meyer zu Schwabedissen, Friederike/Pott, Andreas 2015: Bildungsmigranten in der Region. Instrumente und Handlungsempfehlungen zur Unterstützung des Berufseinstiegs von internationalen Studierenden mit deutschem Hochschulabschluss. IMIS Policy Brief, Osnabrück.

Middendorff, Elke/Apolinarski, Beate/Becker, Karsten/Bornkessel, Philipp/Brandt, Tasso/ Heißenberg, Sonja/Poskowsky, Jonas 2017: Die wirtschaftliche und soziale Lage der Studierenden in Deutschland 2016, Berlin. 
OECD 2017: Nach der Flucht: Der Weg in die Arbeit. Arbeitsmarktintegration von Flüchtlingen in Deutschland, Paris.

Roth, Roland 2015: Willkommensregionen für ausländische Studierende, Gütersloh.

Sachverständigenrat Wirtschaft - Sachverständigenrat zur Begutachtung der gesamtwirtschaftlichen Entwicklung 2017: Für eine zukunftsorientierte Wirtschaftspolitik. Jahresgutachten 2017/18, Wiesbaden.

Sachverständigenrat Wirtschaft 2020: CoronaKrise gemeinsam gewältigen, Resilienz und Wachstum stärken. Jahresgutachten 2020/21, Wiesbaden.

Schammann, Hannes/Younso, Christin 2016: Studium nach der Flucht? Angebote deutscher Hochschulen für Studieninteressierte mit Fluchterfahrung. Empirische Befunde und Handlungsempfehlungen, Hildesheim.

Schreier, Margrit 2014: Varianten qualitativer Inhaltsanalyse: Ein Wegweiser im Dickicht der Begrifflichkeiten, in: Forum: Qualitative Sozialforschung 15: 1.

Seyfeli, Funda/Elsner, Laura/Wannemacher, Klaus 2020: Vom Corona-Shutdown zur Blended University? ExpertInnenbefragung Digitales Sommersemester, Baden-Baden.

Statistics Canada 2021: Postsecondary International Student Enrolments, by Country of Citizenship. (https://www150.statcan.gc.ca/t1/tbl1/en/tv.action?pid=3710018402\#tables, 26.05.2021)

Statistisches Bundesamt 2021a: Bildungsausländer/-innen nach Herkunftsstaaten. Tabelle 2.5.50. (https://www.datenportal.bmbf.de/portal/de/Tabelle-2.5.50.html, 02.09.2021)

Statistisches Bundesamt 2021b: Ausbildungsförderung nach dem Bundesausbildungsförderungsgesetz (BAföG). Fachserie 11 Reihe 7 - 2020. (https:// www.destatis.de/DE/Themen/Gesellschaft-Umwelt/ Bildung-Forschung-Kultur/Bildungsfinanzen-Ausbildungsfoerderung/Publikationen/DownloadsAusbildungsfoerderung/bundesausbildungsfoerderung-2110700207005.html, 13.09.2021)

Stemmer, Petra 2014: Wie kann das Studium besser gelingen? Problembereiche und Erfolgsfaktoren von Bildungsausländern: Eine explorative Studie an der Universität zu Köln, Baden-Baden.
Stifterverband 2020: Hochschulen, Corona und jetzt? Wie Hochschulen vom Krisenmodus zu neuen Lehrstrategien für die digitale Welt gelangen. Future Skills Diskussionspapier 4, Essen.

SVR - Sachverständigenrat für Integration und Migration 2021: Normalfall Diversität? Wie das Einwanderungsland Deutschland mit Vielfalt umgeht. Jahresgutachten 2021, Berlin.

SVR-Forschungsbereich - Forschungsbereich beim Sachverständigenrat deutscher Stiftungen für Integration und Migration 2014: Diskriminierung am Ausbildungsmarkt: Ausmaß, Ursachen und Handlungsperspektiven, Berlin.

SVR-Forschungsbereich 2015: Zugangstor Hochschule. Internationale Studierende als Fachkräfte von morgen gewinnen, Berlin.

SVR-Forschungsbereich 2016a: Sonderauswertung des Datensatzes aus dem Forschungsprojekt Study \& Work, Berlin.

SVR-Forschungsbereich 2016b: Engagiert gewinnt. Bessere Berufschancen für internationale Studierende durch Praxiserfahrungen, Berlin.

SVR-Forschungsbereich 2017a: Vom Hörsaal in den Betrieb? Internationale Studierende beim Berufseinstieg in Deutschland, Berlin.

SVR-Forschungsbereich 2017b: Allein durch den Hochschuldschungel. Hürden zum Studienerfolg für internationale Studierende und Studierende mit Migrationshintergrund, Berlin.

SVR-Forschungsbereich 2019: Dem demografischen Wandel entgegen. Wie schrumpfende Hochschulstandorte internationale Studierende gewinnen und halten, Berlin.

SVR-Forschungsbereich 2020: Zugang per Zufallsprinzip? Neuzugewanderte auf dem Weg in die berufliche Bildung, Berlin.

Taapken, Nelson/Heinen, Markus 2021: Blick in die Zukunft. Wie die Arbeitswelt nach Corona aussieht. Beitrag vom 28.05.2021. (https://www. ey.com/de_de/workforce/arbeiten-nach-coronaneue-beduerfnisse-ernst-nehmen, 02.09.2021)

Übelmesser, Silke/Henn, Sebastian 2019: Hochqualifiziert. International. Thüringen. Abschlussbericht des HiTh-Projekts, Jena. 
Voigt, Anja 2011: Peer Tutoring mit nicht-muttersprachlichen Studierenden am Schreibzentrum der Europa-Universität Viadrina. Eine qualitative Untersuchung auf der Basis von Beratungsprotokollen, Frankfurt/Oder.

Wissenschaftsrat 2016: Empfehlungen zur Gewinnung, Integration und Qualifizierung internationaler Studierender, Köln.

Wissenschaftsrat 2019: Empfehlungen zu hochschulischer Weiterbildung als Teil des lebenslangen Lernens, Berlin. 


\section{B Tabellen}

TABELLE 8: TEILNEHMENDE IN DEN UNTERSUCHTEN INTEGRA- UND PROFI-PROJEKTEN

\begin{tabular}{|c|c|c|}
\hline & Integra & PROFI \\
\hline Projektteilnehmende & 4.233 & 192 \\
\hline \multicolumn{3}{|l|}{ davon: } \\
\hline männlich & $60 \%$ & $61 \%$ \\
\hline weiblich & $40 \%$ & $39 \%$ \\
\hline divers & $0 \%$ & $0 \%$ \\
\hline mit Fluchterfahrung & $55 \%$ & $82 \%$ \\
\hline ohne Fluchterfahrung & $16 \%$ & $14 \%$ \\
\hline keine Angabe & $29 \%$ & $4 \%$ \\
\hline Syrien [Staatsangehörigkeit] & $33 \%$ & $31 \%$ \\
\hline Türkei & $16 \%$ & $29 \%$ \\
\hline Iran & $10 \%$ & $12 \%$ \\
\hline Afghanistan & $4 \%$ & $1 \%$ \\
\hline China & $3 \%$ & $1 \%$ \\
\hline Irak & $3 \%$ & $4 \%$ \\
\hline Indien & $2 \%$ & $1 \%$ \\
\hline Sonstige & $29 \%$ & $21 \%$ \\
\hline
\end{tabular}

Quelle: Eigene Auswertung der Daten aus Integra und PROFI

TABELLE 9: ABLEHNUNGSGRÜNDE BEI BEWERBUNGEN, ANTEILIG AN PROJEKTGEFÖRDERTEN HOCHSCHULEN

Hochschulen, an denen Bewerbungen abgelehnt wurden wegen:

unzureichender Finanzmittel

unzureichender Qualifikation der Bewerbenden 
TABELLE 10: ARBEITSMARKTVORBEREITUNG IN DEN UNTERSUCHTEN INTEGRA- UND PROFI-PROJEKTEN

\begin{tabular}{lrr} 
& Integra & PROFI \\
Gesamtzahl der Einzelangebote & $\mathbf{3 1 6}$ & $\mathbf{1 8 9}$ \\
davon: & & 24 \\
Workshops & 134 & 9 \\
Informationsveranstaltungen & 56 & 13 \\
Coachings & 44 & 110 \\
Seminare/Lehrveranstaltungen & 29 & 4 \\
Mentorenprogramme & 7 & 2 \\
Messen & 6 & 27 \\
sonstiges & 40 & 90 \\
neues Angebot & 179 & 99 \\
bestehendes Angebot & 137 & \\
\hline
\end{tabular}

Anmerkung: Individuelle Beratungs- und Betreuungsangebote, z. B. zur persönlichen Karriereplanung, sind in der Tabelle nicht enthalten. Quelle: Eigene Auswertung der Daten aus Integra und PROFI

TABELLE 11: VERMITTELTE ÜBERGANGSMÖGLICHKEITEN IN DIE ARBEITSWELT

\begin{tabular}{lrr} 
& PROFI \\
Insgesamt & Integra & $\mathbf{4 9}$ \\
davon: & $\mathbf{6 5 7}$ & 14 \\
$\quad$ Vorstellungsgespräche & 146 & 16 \\
Praktika & 90 & 0 \\
Schnuppertage & 50 & 1 \\
Hospitationen & 49 & 12 \\
fachspezifische Beschäftigungen & 51 & 1 \\
nicht fachspezifische Beschäftigungen & 21 & 0 \\
Abschluss- oder Projektarbeiten in Betrieben & 18 & 5 \\
\hline sonstige & 232 & \\
\hline
\end{tabular}

Anmerkung: Die Vermittlungserfolge beziehen sich auf den Zeitraum von Mitte 2020 bis Anfang 2021.

Quelle: Eigene Auswertung der Daten aus Integra und PROFI 
TABELLE 12: INTERVIEW-LEITFADEN FÜR DAS PROJEKTVERANTWORTLICHE HOCHSCHULPERSONAL

\section{Nr. INTERVIEW-FRAGE}

1 Redeanlass:

Was ist Ihre Rolle an der Hochschule mit Blick auf geflüchtete Studierende? HÜRDEN FÜR GEFLÜCHTETE

2 Redeanlass:

Was sind die größten Hindernisse für geflüchtete Studierende, die im regionalen Arbeitsmarkt Fuß fassen möchten? Gibt es bestimmte Gruppen, die es besonders schwer haben?

Folgefragen (falls Interviewte nicht selbst darüber sprechen)

- Wie ist es mit geflüchteten Frauen?

- Gibt es Unterschiede nach Herkunftsregion?

- Gibt es Unterschiede nach Studienfach?

- Gibt es Unterschiede nach angestrebtem Abschluss?

3 Redeanlass:

Sie haben einige Hürden genannt. Inwiefern unterscheiden sich diese von den Hürden anderer Studierender?

Folgefragen:

- Unterschiede zu einheimischen Studierenden?

- Unterschiede zu anderen internationalen Studierenden?

4 Redeanlass:

Was tut Ihre Hochschule, um geflüchteten Studierenden den Weg in den Arbeitsmarkt zu ebnen?

Folgefragen (falls pandemiebedingt bislang nur wenig unternommen wurde)

- Wie hilft Ihnen die Projektförderung dabei?

- Welche Unterstützung erfahren Sie von der Hochschule selbst?

- Welche Folgen hat die Corona-Pandemie auf Ihr Angebot an dieser Stelle?

- Was erwarten Sie hier in den kommenden zwölf Monaten?

5 Redeanlass:

Wie bringen Sie geflüchtete Studierende mit Unternehmen in Kontakt?

Folgefragen:

- Zu wem sonst stellen Sie Kontakt her? Wie machen Sie das? Welche Rolle spielt Service Learning dabei?

6 Redeanlass:

Welche Angebote zum Berufseinstieg finden digital oder im Blended-Learning-Format statt?

Was sind Ihre bisherigen Erfahrungen mit diesen Angeboten?

\section{Folgefragen:}

-Wie ist die Beteiligung im Vergleich zu Präsenzangeboten?

- Wie binden Sie Unternehmen und andere Externe in diese Angebote ein?

- Welche Entwicklungspotenziale sehen Sie hier?

KOOPERATIVES ÜBERGANGSMANAGEMENT

7 Redeanlass:

Wie ich sehe, unternimmt Ihre Hochschule bereits einiges, damit geflüchtete Studierende letztendlich auch den Weg in den Arbeitsmarkt finden. Machen Sie das als Hochschule alles allein? Oder arbeiten Sie auch mit externen Partnern zusammen?

8 Redeanlass:

Wie sieht diese Zusammenarbeit konkret aus?

Folgefragen:

-Wie viele Organisationen sind beteiligt? Wer genau?

- Wie häufig besprechen Sie sich?

- Stimmen Sie Ziele und Maßnahmen miteinander ab?

- Wie wird die Zusammenarbeit koordiniert? Und durch wen?

- Welche Hürden stehen Ihnen im Weg? 
Nr. INTERVIEW-FRAGE

9 Redeanlass:

Wie stellen Sie sich die Zusammenarbeit in Zukunft vor?

Folgefragen:

- Wie soll die Zusammenarbeit koordiniert werden? Und durch wen?

-Woher sollen die Mittel für einen solchen Mehraufwand kommen?

- Welche Vorteile hat diese Art der Zusammenarbeit für Sie? Welche Nachteile?

- Was erwarten Sie hier in den kommenden zwölf Monaten?

\section{Redeanlass:}

Ihre Hochschule liegt in einer Region, in der nur ein kleiner Teil der Bevölkerung einen Migrationshintergrund hat/einer Region, in der zwar viele Menschen einen Migrationshintergrund haben, gewisse Ressentiments gegen Neuzugewanderte aber nicht ausbleiben. Inwiefern spielt dies eine Rolle beim Berufseinstieg der geflüchteten Studierenden?

\section{Folgefragen:}

- Was kann man hier tun?

- Welche Risiken sehen Sie, wenn sich die politische Polarisierung im Land bzw. hier in der Region weiter zuspitzt?

\section{Redeanlass:}

Abschließend gefragt: Was entscheidet letztendlich darüber, ob geflüchtete Studierende nach Abschluss eine angemessene Stelle finden?

Folgefrage:

-Was können und sollen Hochschulen hier leisten?

12 Redeanlass:

Was würde Ihnen nach Projektende am meisten bei Ihrer Arbeit mit geflüchteten Studierenden helfen?

Folgefrage:

- Und vonseiten Ihrer Hochschule?

13 Redeanlass:

Wir haben jetzt einiges besprochen. Möchten Sie noch etwas ergänzen?

Vielen Dank für dieses Interview.

Anmerkung: Die Interviewleitfäden für Projektteilnehmende und externe Partnerorganisationen können auf Anfrage vom wissenschaftlichen Stab des SVR zur Verfügung gestellt werden.

Quelle: Eigene Leitfadenentwicklung 


\title{
Impressum
}

\author{
Herausgeber \\ Deutscher Akademischer Austauschdienst \\ German Academic Exchange Service \\ Kennedyallee 50, D - 53175 Bonn \\ www.daad.de
}

Datenauswertung und Berichtslegung

Sachverständigenrat für Integration und Migration (SVR) gGmbH

Neue Promenade 6

10178 Berlin

Tel.: 030/288 86 59-0

Fax: 030/288 86 59-11

info@svr-migration.de

www.svr-migration.de

Redaktionsschluss: 25. September 2021

\section{Koordination}

Dr. Katharina Fourier (DAAD)

Rachel Estévez Prado (DAAD)

\section{Autorinnen und Autoren}

Simon Morris-Lange, Wissenschaftlicher Mitarbeiter, Wissenschaftlicher Stab des SVR Dr. Mohini Lokhande, Stellvertretende Leiterin, Wissenschaftlicher Stab des SVR Unter Mitarbeit von Mai-Linh Ho Dac, Wissenschaftliche Hilfskraft, SVR

(c) DAAD - Alle Rechte vorbehalten

DOI: 10.46685/DAADStudien.2021.02

Dieses Werk steht unter der Creative Commons Namensnennung - Nicht kommerziell Weitergabe unter gleichen Bedingungen 3.0 Deutschland Lizenz (CC BY-NC-SA 3.0 DE) https://creativecommons.org/licenses/by-nc-sa/3.0/de/ 